\title{
Know Your Neighborhood: A Detailed Model Atmosphere Analysis of Nearby White Dwarfs
}

\author{
N. Giammichele, P. Bergeron ${ }^{1}$, \& P. Dufour \\ Département de Physique, Université de Montréal, C.P. 6128, Succ. Centre-Ville, \\ Montréal, Québec H3C зJ7, Canada. \\ noemi.giammichele@astro.umontreal.ca, pierre.bergeron@astro.umontreal.ca, \\ patrick.dufour@astro.umontreal.ca
}

\begin{abstract}
We present improved atmospheric parameters of nearby white dwarfs lying within $20 \mathrm{pc}$ of the Sun. The aim of the current study is to obtain the best statistical model of the least-biased sample of the white dwarf population. A homogeneous analysis of the local population is performed combining detailed spectroscopic and photometric analyses based on improved model atmosphere calculations for various spectral types including DA, DB, DC, DQ, and DZ stars. The spectroscopic technique is applied to all stars in our sample for which optical spectra are available. Photometric energy distributions, when available, are also combined to trigonometric parallax measurements to derive effective temperatures, stellar radii, as well as atmospheric compositions. A revised catalog of white dwarfs in the solar neighborhood is presented. We provide, for the first time, a comprehensive analysis of the mass distribution and the chemical distribution of white dwarf stars in a volume-limited sample.

Subject headings: Solar neighborhood - stars: luminosity function, mass function - techniques: photometric - techniques: spectroscopic - white dwarfs
\end{abstract}

\section{Introduction}

White dwarf stars represent a significant contribution to the global stellar population and an important indicator of the evolutionary history of the Galaxy. As such, it becomes

\footnotetext{
${ }^{1}$ Visiting Astronomer, Kitt Peak National Observatory, National Optical Astronomy Observatory, which is operated by the Association of Universities for Research in Astronomy (AURA) under cooperative agreement with the National Science Foundation.
} 
crucial to characterize and understand the white dwarf population as fully as possible. Mass distribution, space density, and chemical composition are most valuable pieces of information as we attempt to better constrain the evolutionary history of these stars. Similarly, the white dwarf luminosity function, defined as the number of stars as a function of their intrinsic luminosity, becomes a valuable tool to narrow down the age of the Galactic disk. But in order to take advantage of these indicators, the white dwarf population sampled must be as close as possible to being statistically complete.

The white dwarf population is composed mainly of low-luminosity stars that are rather difficult to study as we move further away from the Sun. Candidates are mainly discovered from either proper-motion-limited or ultraviolet (UV) excess surveys. Proper-motion surveys are characterized by the discovery of high-proper motion stars through a comparison of identical fields observed at two different epochs. By further combining these proper motions with color indices, white dwarfs can be distinguished successfully from other stellar populations. Despite its wide use in the building of large surveys, important flaws remain. Proper-motion surveys naturally present a high kinematic bias. Moreover, high density regions, such as the galactic plane, are usually avoided.

Additional contributions to our knowledge of the white dwarf population were made through UV-excess surveys, a technique primarily used among photometric surveys to identify hot white dwarf candidates. Studies based on UV-excess, magnitude-limited surveys lead to the building of the bright end of the luminosity function, like those recently derived from the Palomar-Green (PG; Liebert et al. 2005; Bergeron et al. 2011) and the Kiso surveys (Limoges \& Bergeron 2010). However, being restricted to the detection of only bluer and thus hotter objects represents a significant bias, and does not allow for a better understanding of the faint end of the luminosity function. The goal of compiling a complete sample is thus highly compromised.

Proper-motion and UV-excess surveys overlap very little and are both incomplete in their own way. The one possibility for obtaining a less biased sample is to use a complete volume-limited sample, centered around the Sun. Such a local sample can provide an accurate statistical model as long as the right balance between high completeness and small number statistics is achieved. A precise picture of this local sample can, if large enough, be extended to the rest of the Galaxy to assess the importance of the white dwarf population to the overall mass budget of the galactic disk.

Numerous studies were performed aiming to complete and to characterize the sample of nearby white dwarfs. The first study dedicated to building a complete census of the local sample of white dwarfs was performed by Holberg et al. (2002). The distance of 20 pc was chosen to correspond to the volume of the NSTARS database, whose goal is to com- 
pile information on all possible stellar sources near the Sun, in order to achieve a better understanding of the local stellar population. As Holberg et al. (2002) further discussed in their work, the volume defined by this 20 pc limit was assumed to be reasonably complete. The determination of the white dwarf candidates was entirely based on photometric magnitudes collected from the Villanova White Dwarf Catalog (McCook \& Sion 1999, hereafter WD Catalog). Two main selection criteria were used to determine white dwarfs within 20 pc. First, the WD Catalog was searched for objects with trigonometric parallaxes $\pi \geq 0$ '.05. For stars with no available parallaxes, the cut was made with photometrically determined distances based on $V-M_{V} \leq 1.505$. Careful attention was paid to remove manually obvious known anomalies. As Holberg et al. (2002) reported, data retrieved in this manner were far from uniform in quality and not homogeneous in any way. A priority scheme was adopted to cope with the different data sources, giving a higher priority to Johnson $B-V$, Strömgren $b-y$, and multichannel $g-r$ color indices, when possible. The direct effect of this selection led to major inhomogeneities in the calculations of absolute visual magnitudes and resulting distances. The subsequent analysis, based on this local sample, mainly focused on the calculation of the local space density and the estimation of the completeness of the sample. Based on the assumption that the 13 pc sample was entirely known, Holberg et al. (2002) estimated the 20 pc sample to be only $65 \%$ complete. Few details showing the atmospheric properties of the white dwarfs in the solar neighborhood were presented at that time.

The quest for completeness of the local white dwarf sample, based on the 109 candidates determined by Holberg et al. (2002), was pursued through the contributions of Vennes \& Kawka (2003), Kawka et al. (2004), and Kawka \& Vennes (2006), who surveyed the revised NLTT catalog of Salim \& Gould (2003). By using color-color and reduced proper motion diagrams, as well as a spectroscopic follow-up of white dwarf candidates, several stars were added to the original local sample, while some others were removed. In particular, Kawka \& Vennes (2006) extended the search for possible candidates by spectroscopically identifying 8 new white dwarfs lying within 20 pc. Other contributions from Farihi et al. (2005), Subasavage et al. (2007), and Subasavage et al. (2008) are also worth mentioning in this effort.

Holberg et al. (2008) and Sion et al. (2009) reanalyzed the white dwarfs in the solar neighborhood by updating the local sample of Holberg et al. (2002) with the recent discoveries mentioned above, to form a sample composed of 132 stars. Holberg et al. (2008) also compiled atmospheric parameters of the local sample candidates, collected from numerous sources, to calculate the mean mass of the sample, and used a variety of spectroscopic, photometric, and trigonometric distances to better estimate the local space density. Sion et al. (2009) strictly focused on the kinematical properties and the distribution of spectroscopic subtypes of the white dwarf population within 20 pc. 
The lack of consistency between the different model atmospheres and analysis methods makes previous determinations of the ensemble properties of the local white dwarf sample quite uncertain, and may lead to erroneous estimates. Obviously, the quest for completeness of the local sample is still a central preoccupation, but a proper analysis of such a sample has been left aside, and to this day, there has not been any systematic model atmosphere analysis of all available data. It thus appears appropriate, at this time, to revisit the nearby white dwarf population by performing a rigorous analysis, in an homogeneous fashion, of every star in the sample.

In this paper, we present improved atmospheric parameters of all possible nearby white dwarfs lying within 20 pc of the Sun. A homogeneous and complete analysis of the local population is performed combining detailed spectroscopic and photometric analyses based on improved model atmosphere calculations for various spectral types including DA, DC, DQ, and DZ stars. Our photometric and spectroscopic observations are presented in Section 2 , while the theoretical framework is discussed in Section 3. The photometric and spectroscopic data are then analyzed in Sections 4 and 5, respectively. Selected astrophysical results from our analysis, including the mass distribution and luminosity function, are examined in Section 6, and our conclusions follow in Section 7 .

\section{Observational Data}

\subsection{Definition of the Local Sample}

The sample we analyze in this study is composed of spectroscopically identified white dwarfs that lie in the solar neighborhood, within the approximate limit defined at $20 \mathrm{pc}$. It is mostly drawn from the complete list presented in Sion et al. (2009), an updated version of the local population defined by Holberg et al. (2002, 2008). As mentioned earlier, significant additions to this initial sample have been made by Kawka et al. (2004), Kawka \& Vennes (2006), and Subasavage et al. (2007, 2008), with some contributions from other studies (see references in Sion et al. 2009). We increased the sample size by taking into account all possible white dwarfs that could lie within the uncertainties inside the 20 pc region, which means including all objects from Table 4 of Holberg et al. (2008). We also include the peculiar DQ star LHS $2229(1008+290)$ since a new trigonometric parallax was made available to us by H. C. Harris. (2010, private communication) places the star inside the 20 pc region, and the DC star LHS 1247 (0123-262) since its distance, estimated from the photometric observations of Bergeron, Ruiz, \& Leggett (1997, hereafter BRL97), places this candidate inside our region of interest, within the uncertainties. 
We also include two DA stars from the spectroscopic analysis of Gianninas et al. (2011), L796-10 (0053-117) and GD 25 (0213+396), whose distances are estimated to be inside the 20 pc region, within the uncertainties; G138-31 (1625+093) also falls into this category although the trigonometric parallax measurement available for this star places it beyond $20 \mathrm{pc}$ (note that we considered here only stars that were hot enough for the spectroscopic method to be reliable, $T_{\text {eff }} \gtrsim 6500 \mathrm{~K}$ ). There are also several DB stars in the spectroscopic analysis of Bergeron et al. (2011) with distances within 20 pc, although all of these lie in a temperature regime where the physics of line broadening becomes more questionable, van der Waals broadening in particular. The closest DB star with reliable atmospheric parameters lies at a distance of $\sim 30 \mathrm{pc}$.

Finally, an initial sample of 169 white dwarf candidates has been retained for this analysis. The complete list of objects is presented in Table 1 where we give for each star the WD number from the WD Catalog as well as an alternate name; whenever possible we used the LHS or the Giclas names unless the object is better known under another name in the literature. The additional entries for each object are described in the next section.

\subsection{Spectroscopic Observations}

One of the original goals of this project was to characterize, as best we could, the white dwarf population in the solar neighborhood. This requires us to first provide a spectroscopic snapshot of this population in the form of an atlas similar to that published by Wesemael et al. (1993). All in all, we managed to secure high signal-to-noise ratio spectroscopic observations for 166 objects in our local sample (the three objects missing are the Sirius-like systems 0208-510, 0415-594, and 1132-325). We describe these observations in turn.

Most of the blue spectra $(\lambda \sim 3700-5200 \AA)$ for the DA white dwarfs were already available to us from of our numerous studies of these stars (see, e.g., Bergeron et al. 1992a; Liebert et al. 2005; Gianninas et al. 2011), while several spectra covering the region near $\mathrm{H} \alpha$ - required to constrain the atmospheric composition of the coolest degenerates - were taken from the studies of BRL97 and Bergeron, Leggett, \& Ruiz (2001, hereafter BLR01). Additional spectra were also acquired for the specific purpose of this project. For instance, blue spectra were secured during several observing runs at the Steward Observatory $2.3 \mathrm{~m}$ telescope equipped with the Boller \& Chivens spectrograph and a Loral CCD detector. The 4 ..5 slit together with the $600 \mathrm{l} \mathrm{mm}^{-1}$ grating in first order provided a spectral coverage from about 3200 to $5300 \AA$ at an intermediate resolution of $\sim 6 \AA$ FWHM. Additional spectra covering the red portion of the spectrum were acquired with the same setup but with the 
$400 \mathrm{l} \mathrm{mm}^{-1}$ grating in first order allowing a spectral coverage from about 3700 to $6900 \AA$ at a resolution of $\sim 9 \AA$ FWHM. Similar spectra with a coverage from 3800 to $6700 \AA$ were also secured at the Kitt Peak National Observatory $2.1 \mathrm{~m}$ and $4 \mathrm{~m}$ telescopes equipped with the Goldcam and RC spectrographs, respectively. Both used a 2".0 slit with a resolution of $\sim 6 \AA$ FWHM, but different gratings of $316 \mathrm{l} \mathrm{mm}^{-1}$ and $500 \mathrm{l} \mathrm{mm}^{-1}$, respectively. Details of our observing and reduction procedures can be found in Saffer et al. (1994).

We also make use of several spectra already published in the literature, and generously made available to us by the authors. This is the case for white dwarfs discovered in the ongoing survey by J. Subasavage: LHS 1243 (0121-429), LP 593-56 (0344+014), SCR 0821-6703 (0821-669), SCR 2012-5956 (2008-600), and L570-26 (2138-332) from Subasavage et al. (2007); LP 50-73 (0011-721), L454-9 (0655-390), SCR 0708-6706 (0708-670), SCR 0753-2524 (0751-252), SCR 0818-3110 (0816-310), and SCR 1118-4721 (1116-470) from Subasavage et al. (2008); LEHPM 2-220 (1009-184) from Subasavage et al. (2009); L40-116 (1315-781) from J. P. Subasavage (2010, private communication). From the NLTT Survey: LHS 1421 (NLTT 8435, 0233-242), LP 522-46 (NLTT 56805, 2322+137), and G36-29 (NLTT 8581, 0236+259) from Vennes \& Kawka (2003); LP 872-20 (NLTT 49985, 2048-250) from Kawka et al. (2004); LP 294-61 (NLTT 3915, 0108+277) from Kawka \& Vennes (2006). And from other studies: LSR $1817+1328(1814+134)$ from Lépine et al. (2003); PM J13420-3415 (1339-340) from Lépine et al. (2005); SSPM J2231-7514 (2226-754) and SSPM J2231-7515 (2226-755) from Scholz et al. (2002); LEHPM 1-4466 (2211-392) from Oppenheimer et al. (2001); LHS 1008 (0000-345) from Reimers et al. (1996). Finally, optical spectra for LHS $2229(1008+290)$ and GD $184(1529+141)$ were taken from previous Data Releases of the Sloan Digital Sky Survey.

The spectral type of each white dwarf in our nearby sample is reported in Table 1. In summary, this sample breaks down into the following spectral types: 113 DA, 26 DC, 19 DQ, 10 DZ and 1 DBQA. Strangely enough, there is not a single warm $\left(T_{\text {eff }}>13,000 \mathrm{~K}\right)$ DB star in this nearby sample. As discussed above, according to the spectroscopic analysis of relatively bright DB white dwarfs of Bergeron et al. (2011), the closest DB star lies at a distance of $\sim 30 \mathrm{pc}$.

Figure 1 presents the blue spectra of DA and DAZ stars in our sample in order of decreasing effective temperature (determined below in Section 51). The spectrum of LHS 1660 (0419-487) is obviously contaminated by the presence of an M dwarf companion. GR 431 (0939+071; last object in Figure1), also known as PG 0939+072, is a problematic object. Classified DC7 in the PG catalog, it was not included in the spectroscopic analysis of the DA white dwarfs identified in the PG survey (Liebert et al. 2005), despite the fact that it had been reclassified as DA2 in Holberg et al. (2002). However, it appeared again as DC7 in 
Table 4 of Holberg et al. (2008), a list of possible white dwarfs within 20 pc. Our spectrum shows that GR 431 is simply not a white dwarf star (reclassified as a main sequence dF star by Gianninas et al. 2011), and it is therefore excluded from our analysis. Our nearby sample thus includes 168 genuine white dwarf stars, some of which are unresolved double degenerate systems.

The blue spectra of DA and DAZ stars too cool to be analyzed using line profile fitting techniques are displayed in Figure 2. In some cases, these objects are completely featureless in the spectral region shown here, and the presence of hydrogen can only be inferred by the detection of $\mathrm{H} \alpha$. DAZ stars in Figures 1 and 2 can be easily recognized by the presence of the Ca II H and K lines, the most notable in this sample being GD 362 (1729+371; Figure 1) whose spectrum also shows spectral lines from Ca I, Mg I, and Fe I (Gianninas et al. 2004); high-resolution spectroscopy actually reveals the presence of 17 different elements, including helium (Zuckerman et al. 2007). Note that our blue spectrum of LP 294-61 (0108+277; Figure 21) does not reveal any metallic feature and we have thus reclassified this star as DA (see also Section 3.6 of Farihi et al. 2009).

The DA (and DZA) stars in our sample for which spectra at $\mathrm{H} \alpha$ are also available are presented in Figure 3 as a function of decreasing equivalent widths. The presence of $\mathrm{H} \alpha$ in the coolest white dwarfs is crucial to better constrain the atmospheric parameters using the photometric method described in Section 4, the coolest DA stars in which $\mathrm{H} \alpha$ can be detected in our sample have photometric temperatures around $T_{\text {eff }} \sim 5000 \mathrm{~K}$. The Zeeman triplet is clearly visible in five magnetic DA stars displayed in the right panel of Figure 3. Putney (1997) classified G234-4 (0728+642) as DAP based on polarization data at $\mathrm{H} \alpha$, which indicated a $3 \sigma$ detection with $B_{e}=+39.6 \pm 11.6 \mathrm{kG}$, and a suggestion of Zeeman features. Our spectrum of G234-4 shows no evidence of magnetic splitting due to the lower spectral resolution of our data.

Our set of DC white dwarfs are displayed in Figure 4 in order of right ascension. These have featureless spectra, even at $\mathrm{H} \alpha$. The absence of any absorption feature implies that they are either cool $\left(T_{\text {eff }} \lesssim 10,000 \mathrm{~K}\right)$ helium-atmosphere white dwarfs, or hydrogen-atmosphere white dwarfs too cool to show $\mathrm{H} \alpha\left(T_{\text {eff }} \lesssim 5000 \mathrm{~K}\right)$. Only a detailed photometric analysis of these stars can resolve this ambiguity. LHS 1008 (0000-345), classified DC by BRL97 but DAH by Reimers et al. (1996), is not displayed here and is further discussed below.

Spectroscopic data of normal DQ stars in our sample are displayed in Figure 5, Because of the spectral classification scheme devised by McCook \& Sion (1999), DQ white dwarfs may have carbon features detectable only in the ultraviolet. The spectrum of L97-3 (0806-661), for instance, appears featureless in the optical. G47-18 $(0856+331)$ is a unique DQ star (excluding those discovered in the SDSS) that shows both $\mathrm{C}_{2}$ Swan bands and $\mathrm{C}$ I atomic 
lines. Note also the presence of the CH G-band $(\sim 4300 \AA)$ in the spectrum of G99-37 (0548-001) and BPM 27606 (2154-512), the only two such stars known; both stars are also magnetic and show circular polarization in the $\mathrm{CH}$ band (Vornanen et al. 2010, and references therein). BPM 27606 also turns out to be one of the normal DQ stars with the strongest $\mathrm{C}_{2}$ Swan bands known. In addition to a very weak $\mathrm{C}_{2}$ absorption feature, the spectrum of GD $184(1529+141)$ also shows narrow hydrogen lines (see also Figure 31), up to $\mathrm{H} \gamma$, which would make this star a unique DAQ white dwarf. Our detailed analysis of this object, described below, reveals instead that GD 184 is in fact a DA + DQ double degenerate system.

Peculiar DQ stars with shifted $\mathrm{C}_{2}$ Swan bands are displayed in Figure 6; a normal DQ star is also reproduced at the top of the figure for comparison. Note how the molecular bands in the other objects appear shifted and more symmetrical with respect to this normal DQ star, with the extreme case of LHS $2229(1008+290)$ displayed at the bottom. This phenomenon has recently been explained by Kowalski (2010) as a result of pressure shifts of the carbon bands that occurs in cooler, helium-dominated atmospheres. These stars are now being classified as DQpec (rather than $\mathrm{C}_{2} \mathrm{H}$ stars). The presence of a very strong magnetic field has also been reported in the bottom two objects (see, e.g., Schmidt et al. 1999).

Our DZ spectra, showing the presence of metal lines, mainly the Ca II H \& K doublet, are displayed in Figure 7. L745-46A (0738-172), GD 95 (0843+358), and Ross 640 (1626+368) are actually DZA stars with very shallow $\mathrm{H} \alpha$ absorption lines (shown in Figure 3), resulting from the presence of a trace of hydrogen in a helium-dominated atmosphere. LP 701-29 (2251-070) is a heavily blanketed DZ star, the only known case where Ca I $\lambda 4226$ appears stronger than the Ca II doublet.

Finally, additional spectra of miscellaneous white dwarfs are displayed in Figure8, These include LDS 678A (1917-077), the only cool and relatively bright DBQA star known (carbon is observed only in the UV), GW+70 $8247(1900+705)$, a heavily magnetic DA white dwarf, and G240-72 (1748+708) whose spectrum is characterized by a deep yellow sag of unknown origin in the 4400-6300 Å region (see also Wesemael et al. 1993). Our spectrum of LDS 678A shows a significant absorption feature at $\mathrm{H} \alpha$, although another spectrum taken several years earlier showed only a hint of $\mathrm{H} \alpha$, which might indicate some spectroscopic variability; the only other features present in the spectrum are weak neutral helium lines (the feature observed at $\sim 6300 \AA$ is a night sky feature). Also shown in Figure 8 are two spectra of LHS 1008 (0000-345), classified DC by BRL97 based on the top spectrum displayed here. However, spectroscopic observations analyzed by Reimers et al. (1996), and reproduced at the bottom of Figure 8, clearly indicate the presence of magnetic features, suggesting that LHS 1008 might be variable. Reimers et al. (see their Figure 6) actually managed to reproduce the 
absorption feature near $4600 \AA$ with a magnetic offset dipole, hydrogen-atmosphere model, and a polar field strength of 86 MG. Finally, G227-35 (1829+547) is another magnetic white dwarf with strong magnetic polarization (Angel et al. 1975). Cohen et al. (1993) also reported a prominent feature near $7450 \AA$ attributed to the stationary point in a transition of $\mathrm{H} \alpha$. In the wavelength range displayed in Figure 8, however, the spectrum of G227-35 appears featureless, with the exception perhaps of a weak absorption feature near $6000 \AA$, which is actually predicted in the models for this star shown in Figure 7 of Putney \& Jordan (1995).

A final quick glance at the spectra shown in the figures above reveals that the local population of white dwarf stars includes some of the strangest and more unique objects we know. We definitely live in a strange neighborhood!

\subsection{Photometric Observations}

Optical BVRI photometry was retrieved from the studies of BRL97 and BLR01 for 82 cool white dwarfs in our sample. Additional $V R I$ photometry for 21 objects was taken from the various studies of nearby white dwarfs in the southern hemisphere by J. Subasavage. For other white dwarfs in our sample with no available $(B) V R I$ photometry, we relied instead on Strömgren ubvy photometry (3 objects), SDSS ugriz photometry (7 objects), multichannel data (1 object), or additional sources given in Table 1 . In order to characterize the complete energy distribution of cool white dwarfs, the optical photometry was combined with infrared $J H K$ photometry from BRL97 and BLR01 (75 objects), $J H K_{S}$ photometry extracted from the online version of the Two Micron All Sky Survey (2MASS) survey (39 objects), or JHK photometry on the MKO system taken from Kilic et al. (2006, 2 objects). For LHS 2229 $(1008+290)$, we used the BVIJHK photometry from Schmidt et al. (1999).

Our adopted optical and infrared photometric data are reported in Table 1 together with references given in the last column; a special column also indicates which optical photometric set is used. For stars analyzed only spectroscopically, we also provide $V$ magnitudes taken mostly from the online version of the WD Catalog, which will serve to estimate spectroscopic distances. Photometric uncertainties are taken from the appropriate references, with the exception of the $V$ magnitudes retrieved from the WD Catalog, for which we assume $5 \%$ for simplicity.

The $(V-I, V-K)$ two-color diagram is displayed in Figure 9 for 99 white dwarfs in our sample with these color indices available. DA and non-DA stars are represented by filled and open circles, respectively. Also shown are the predictions from pure hydrogen and 
pure helium cooling sequences at $\log g=8.0$ (described in Section 3). DA and non-DA stars form two, nearly distinct sequences in this diagram, which follow closely the behavior of the model sequences at higher temperatures, although both sequences are shown to cross at lower temperatures. Hence, the atmospheric composition of the coolest non-DA stars in this diagram cannot be interpreted easily. For instance, ER 8 (1310-472; labeled in the figure) is the coolest and oldest white dwarf identified in the analysis of BRL97, and it has a pure hydrogen atmospheric composition despite its DC nature. Worth mentioning here is the strong deficiency of non-DA stars in a particular range of $V-K$ colors between $\sim 1.2$ and 1.7, which corresponds to the so-called non-DA gap first discussed by BRL97. The only non-DA star lying in this interval is L40-116 (1315-781; labeled in the figure); this object will be further discussed in Section 4.2. There are also several outliers identified in this diagram, all of the non-DA type: LHS 2229 (1008+290) and LP 790-29 (1036-204) are DQpec white dwarfs with strong shifted $\mathrm{C}_{2}$ Swan bands (see Figure 6) that affect the colors; LHS 1126 (0038-226) and SCR 2012-5956 (2008-600) both show a strong infrared flux deficiency that has been interpreted in terms of the $\mathrm{H}_{2}$-He collision-induced absorptions in a mixed

H/He atmosphere (see, e.g., Bergeron et al. 1994); G195-19 (0912+536) is a magnetic white dwarf with $\mathrm{a} \sim 100 \mathrm{MG}$ field that probably affects the $I$ passband (see our photometric fit below).

\subsection{Trigonometric Parallax Measurements}

BRL97 found that even though the model energy distributions are somewhat sensitive to surface gravity, it is practically impossible to determine $\log g$ from the observed photometry alone. Only for stars with available trigonometric parallax measurements is it possible to determine the stellar radius, and thus the mass through the mass-radius relation. In our sample, 125 stars have trigonometric parallax measurements taken mostly from the Yale Parallax Catalog (van Altena et al. 1994, hereafter YPC) and the Hipparcos Catalog (Perryman et al. 1997). The parallax values and corresponding uncertainties are reported in Table 1 together with the appropriate reference.

The $M_{V}$ versus $(V-I)$ color-magnitude diagram obtained using these trigonometric parallaxes is displayed in Figure 10 for 85 stars in our sample with available $V$ and $I$ colors. Again, white dwarfs are distinguished in terms of their DA or non-DA spectral types, and the predictions from pure hydrogen and pure helium cooling sequences at $\log g=8.0$ are superimposed on the observed data; note that the data already suggest a slightly higher mean $\log g$ value for this sample. As mentioned by BLR01, DA and non-DA stars form well-defined narrow sequences in this diagram, although not as narrow as those observed in 
the previous figure, most likely because the trigonometric parallax measurements come from inhomogeneous parallax samples. Also, all overluminous white dwarfs hot enough for $\mathrm{H} \alpha$ to be detected are of the DA spectral type. As discussed in BRL97, most, if not all, of these objects are unresolved binaries — e.g., L870-2 (0135-052) — and their luminosity reflects the contribution of two white dwarfs with probably average masses.

\section{Theoretical Framework}

Our model atmospheres and synthetic spectra are derived from the LTE model atmosphere code originally described in Bergeron et al. (1995a) and references therein, with recent improvements discussed in Tremblay \& Bergeron (2009). In particular, we now rely on their improved calculations for the Stark broadening of hydrogen lines with the inclusion of nonideal perturbations from protons and electrons - described within the occupation probability formalism of Hummer \& Mihalas (1988) — directly inside the line profile calculations. Convective energy transport is taken into account following the revised ML $2 / \alpha=0.8$ prescription of the mixing-length theory (see Bergeron et al. 1995b and Tremblay \& Bergeron 2009 for details). Non-LTE effects are also included at higher effective temperatures but these are totally irrelevant for the purpose of this work. More details regarding our heliumatmosphere models are provided in Bergeron et al. (1995a, 2011).

Our model grid covers a range of effective temperature between $T_{\text {eff }}=1500 \mathrm{~K}$ and $45,000 \mathrm{~K}$ in steps of $500 \mathrm{~K}$ for $T_{\text {eff }}<15,000 \mathrm{~K}, 1000 \mathrm{~K}$ up to $T_{\text {eff }}=18,000 \mathrm{~K}, 2000 \mathrm{~K}$ up to $T_{\text {eff }}=30,000 \mathrm{~K}$, and by steps of $5000 \mathrm{~K}$ above. The $\log g$ ranges from 6.5 to 9.5 by steps of 0.5 dex, with additional models at $\log g=7.75$ and 8.25. Additional models, in particular for cool stars, have been calculated with mixed hydrogen and helium compositions of $\log \mathrm{H} / \mathrm{He}=-1.0$ to 3.0 (by steps of 0.5 ).

Synthetic colors are then obtained using the procedure outlined in Holberg \& Bergeron (2006) based on the Vega fluxes taken from Bohlin \& Gilliland (2004). Since the photometric technique described below relies heavily on the flux at the $B$ bandpass, we now include in our models the opacity from the red wing of Ly $\alpha$ calculated by Kowalski \& Saumon (2006) and kindly provided to us by P. Kowalski, which is known to affect the flux in the ultraviolet region of the energy distribution. These improved synthetic colors were used in Figures 9 and 10, and are available from our Website2.

The photometric analysis of DQ and DZ white dwarfs requires improved models since

\footnotetext{
${ }^{2}$ See http://www.astro.umontreal.ca/ bergeron/CoolingModels.
} 
strong carbon or other metallic features may not be the only elements that affect the flux in some photometric bands. Indeed, the presence of heavier elements in helium-rich atmospheres may also provide enough free electrons to affect the atmospheric structure significantly, and thus the predicted energy distributions. To circumvent these problems, we rely on the LTE model atmosphere calculations developed by Dufour et al. (2005, 2007) for the study of DQ and DZ stars, respectively, based on a modified version of the code described in Bergeron et al. (1995a). The main addition to the models is the inclusion of metals and molecules in the equation of state and opacity calculations.

\section{Photometric Analysis}

\subsection{General Procedure}

Atmospheric parameters, $T_{\text {eff }}$ and $\log g$, and chemical compositions of cool white dwarfs can be measured accurately using the photometric technique developed by BRL97. We first convert optical and infrared photometric measurements into observed fluxes and compare the resulting energy distributions with those predicted from our model atmosphere calculations. To accomplish this task, we first transform every magnitude $m$ into an average flux $f_{\lambda}^{m}$ using the equation

$$
m=-2.5 \log f_{\lambda}^{m}+c_{m}
$$

where

$$
f_{\lambda}^{m}=\frac{\int_{0}^{\infty} f_{\lambda} S_{m}(\lambda) \lambda d \lambda}{\int_{0}^{\infty} S_{m}(\lambda) \lambda d \lambda}
$$

and where $S_{m}(\lambda)$ is the transmission function of the corresponding bandpass, $f_{\lambda}$ is the monochromatic flux from the star received at Earth, and $c_{m}$ is a constant to be determined. The transmission functions in the optical for the BVRI, ugriz, and Strömgren photometric systems are described in Holberg \& Bergeron (2006) and references therein, while the multichannel photometric system is discussed in Greenstein (1976). Note that the ugriz and multichannel photometry are defined on the AB magnitude system, which requires a slightly different definition of the above equations (see equation 3 of Holberg \& Bergeron 2006 for instance). The transmission functions for the $J H K$ or $J H K_{S}$ filters on the Johnson-Glass, 2MASS, and Mauna Kea Observatories (MKO) photometric systems are taken respectively from Bessell \& Brett (1988), Cohen et al. (2003), and Tokunaga et al. (2002). Since the 
JHK magnitudes in Table 1 from BRL97 and BLR01 are on the CIT system, they first need to be transformed on the Johnson-Glass system using the equations given by Leggett (1992).

The constants $c_{m}$ in equation (1) for each passband are determined using the improved calibration fluxes from Holberg \& Bergeron (2006), defined with the Hubble Space Telescope absolute flux scale of Vega (Bohlin \& Gilliland 2004), and appropriate magnitudes on a given system. Note that the Strömgren $u$ magnitude in Table 1 of Holberg \& Bergeron (2006) is erroneous and should read 1.437 instead of 1.357 (Hauck \& Mermilliod 1998), which yields a corrected zero point of $c_{u}=-19.80809$ (instead of -19.8882).

For each star in Table 1, a minimum set of four average fluxes $f_{\lambda}^{m}$ is obtained, which can be compared with model fluxes. Since the observed fluxes correspond to averages over given bandpasses, the monochromatic fluxes from the model atmospheres need to be converted into average fluxes as well, $H_{\lambda}^{m}$, by substituting $f_{\lambda}$ in equation 2 for the monochromatic Eddington flux $H_{\lambda}$. We can then relate the average observed fluxes $f_{\lambda}^{m}$ and the average model fluxes $H_{\lambda}^{m}$ — which depend on $T_{\text {eff }}, \log g$, and chemical composition - by the equation

$$
f_{\lambda}^{m}=4 \pi(R / D)^{2} H_{\lambda}^{m}
$$

where $R / D$ defines the ratio of the radius of the star to its distance from Earth. We then minimize the $\chi^{2}$ value defined in terms of the difference between observed and model fluxes over all bandpasses, properly weighted by the photometric uncertainties. Our minimization procedure relies on the nonlinear least-squares method of Levenberg-Marquardt (Press et al. 1986), which is based on a steepest decent method. Only $T_{\text {eff }}$ and the solid angle $\pi(R / D)^{2}$ are considered free parameters, while the uncertainties of both parameters are obtained directly from the covariance matrix of the fit.

For stars with known trigonometric parallax measurements, we first assume a value of $\log g=8$ and determine the effective temperature and the solid angle, which combined with the distance $D$ obtained from the trigonometric parallax measurement, yields directly the radius of the star $R$. The radius is then converted into mass using evolutionary models similar to those described in Fontaine et al. (2001) but with $\mathrm{C} / \mathrm{O}$ cores, $q(\mathrm{He}) \equiv \log M_{\mathrm{He}} / M_{\star}=$ $10^{-2}$ and $q(\mathrm{H})=10^{-4}$, which are representative of hydrogen-atmosphere white dwarfs, and $q(\mathrm{He})=10^{-2}$ and $q(\mathrm{H})=10^{-10}$, which are representative of helium-atmosphere white dwarfs. In general, the $\log g$ value obtained from the inferred mass and radius $\left(g=G M / R^{2}\right)$ will be different from our initial guess of $\log g=8$, and the fitting procedure is thus repeated until an internal consistency in $\log g$ is reached. For white dwarfs with no parallax measurement, we simply assume a value of $\log g=8.0$. 


\subsection{Analysis with Hydrogen- and Helium-Atmosphere Models}

We first perform a detailed analysis of all the objects in our sample with available photometry using hydrogen- and helium-atmosphere models; a more detailed analysis of the DQ and DZ stars in this sample will be presented in the following section. We exclude LHS 1660 (0419-487) from this analysis because of a bright, nearby star that contaminates the photometric observations, and we simply rely on the spectroscopic solution for this object.

Our results for the analysis of the optical and infrared photometry are presented in Figure 11. Average observed fluxes in the left panels are represented by error bars, while the corresponding model fluxes are shown as open or filled circles, depending on the atmospheric composition. The photometric bandpasses used in the fitting procedure and the atmospheric parameters of each solution are indicated in each panel. On the right panels are shown the spectroscopic observations near $\mathrm{H} \alpha$ compared to the model predictions assuming the pure hydrogen solution (the spectral type is also given); these only serve as an internal check of our photometric solutions and are not used in the fitting procedure. For instance, cases where an $\mathrm{H} \alpha$ absorption feature is predicted but is not observed clearly suggest that the pure helium solution is more appropriate. In cases where the star is too cool to show $\mathrm{H} \alpha\left(T_{\text {eff }} \lesssim 5000 \mathrm{~K}\right)$, however, one has to rely on the predicted energy distributions to decide which atmospheric composition best fit the photometric data. Based on our inspection of these fits, we adopt the solutions shown in red in the left panels. In general, the fits to the energy distributions, and the internal consistency with the presence or absence of $\mathrm{H} \alpha$, are excellent. Note that the absorption feature seen in G47-18 $(0856+331)$ is a neutral carbon line and not $\mathrm{H} \alpha$. We discuss some of these photometric fits in turn.

LHS 1008 (0000-345) and G83-10 (0423+120) belong to this strange class of objects, first identified by BRL97, whose energy distributions are better fit with pure hydrogen models (or something intermediate between hydrogen and helium), while their spectra are featureless near the $\mathrm{H} \alpha$ region. Note how the photometric fits for these two objects are qualitatively identical. BRL97 discuss some exotic scenarios to account for this behavior, although none have been successful so far (see, e.g., Malo et al. 1999). The analysis of Reimers et al. (1996) suggests, however, that the feature observed in their spectrum of LHS 1008 (reproduced at the bottom of our Figure 8 ) is due to hydrogen in the presence of a high magnetic field ( $\sim 86$ $\mathrm{MG}$ ). The presence of a strong magnetic field could thus provide a natural explanation for the discrepancy observed here, and magnetic models are probably required to reproduce the

photometry more adequately. If our interpretation is correct, G83-10 could perhaps harbor a strong magnetic field, although the spectropolarimetric survey of DC stars by Putney (1997) reports a null result for this star.

There are five weakly magnetic white dwarfs displayed in Figure 11 that exhibit the Zee- 
man triplet: LHS 1044 (0011-134), LHS 1243 (0121-429), LHS 1734 (0503-174), G99-47 $(0553+053)$, and G256-7 (1309+853). The predicted $\mathrm{H} \alpha$ profiles shown here do not include the magnetic field, however; synthetic spectra with offset dipole models are illustrated in Bergeron et al. (1992b) for instance. In all these cases, the presence of a weak magnetic field does not appear to affect significantly the energy distributions, which are well reproduced by our pure hydrogen models. The same conclusion applies to LHS $1038(0009+051)$, one of the first white dwarfs discovered with a magnetic field below $100 \mathrm{kG}$ (Schmidt \& Smith 1994), which manifests itself here as a small discrepancy in the core of $\mathrm{H} \alpha$. Other magnetic white dwarfs in our sample include G240-72 (1748+708) and G227-35 (1829+547), both classified as DXP stars, with broad, unidentified absorption features. Since we do not have appropriate models for these stars, we simply assume a pure helium composition and we also neglect the $V$ bandpass for G240-72, which is affected by the deep yellow sag observed in Figure 8 .

LHS 1126 (0038-226) and SCR 2012-5956 (2008-600) are the only two objects in our sample that show an infrared flux deficiency (see also Figure 9). As explained above, this flux deficiency is the result of the collision-induced absorption by molecular hydrogen due to collisions with neutral helium, and mixed compositions of $\log \mathrm{H} / \mathrm{He} \sim-1.3$ and -3 , respectively, are required to reproduce their energy distributions. Interestingly enough, both stars appear to have the same $\log g$ values, and thus stellar masses $\left(\sim 0.44 M_{\odot}\right)$.

Our pure helium models obviously fail to reproduce the flux in the $B$ bandpass of the strongest DZ stars in our sample, due to the strong Ca II H \& K absorption lines, or other absorption features in the blue. This is the case for vMa 2 (0046+051), G139-13 (1705+030), and LP 701-29 (2251-070). For these stars in particular, we have simply neglected the $B$ magnitude in our fitting procedure. Note the higher $\log g$ values (up to $\sim 8.4$ ) measured for these stars based on our pure helium models. A similar conclusion applies to the normal DQ stars - for instance LHS 1227 (0115+159), Wolf 219 (0341+182), L879-14 (0435-088), although in most cases, the $\mathrm{C}_{2}$ Swan bands are not strong enough to affect significantly the flux in any particular bandpass. The presence of additional free electrons is affecting the atmospheric structure, however. Note again the high $\log g$ values measured for the DQ stars based on our pure helium models. DZ and DQ white dwarfs will be analyzed in greater detail in the next section using more appropriate models.

Four objects in our sample have only photographic magnitudes in the optical - LP 294-61 (0108+277), G36-29 (0236+259), LP 872-20 (2048-250), and LP 872-20 (2215+368) - and the fits to their energy distributions are not particularly good in this spectral region, although the match at $\mathrm{H} \alpha$ is excellent, with the glaring exception of LP 294-61, which shows a much weaker $\mathrm{H} \alpha$ absorption feature than predicted. Farihi et al. (2009, see their Section 3.6) describes this object as an optical pair of stars composed of a white dwarf and a background 
red dwarf. The two components are still well separated, however, and it is therefore unlikely that the optical and/or infrared magnitudes of the white dwarf are contaminated by the red dwarf. Actually, experiments with only optical or infrared magnitude sets yield the same value of $T_{\text {eff }}$. Most likely, LP 294-61 is an unresolved double degenerate DA + DC system, but given the problems discussed above, the nature of this object remains uncertain.

About 14 white dwarfs in Figure 11 have $\log g$ values and corresponding masses too low to have evolved as single stars within the lifetime of our Galaxy $\left(M \lesssim 0.47 M_{\odot}\right)$. Since with the photometric technique, it is really the radius of the star that is being measured (see equation 3), the most obvious explanation is that these objects are in fact unresolved double degenerates, with two stars contributing to the total luminosity. Indeed, some are well confirmed double degenerate systems: G1-45 (0101+048), L870-2 (0135-052), and L58777A (0326-273). For this last object, the binary nature can also be inferred from the discrepant fit at $\mathrm{H} \alpha$. Such discrepancies are also observed for L532-81 (0839-327) and G187-8 (2048+263), although the binary nature of these stars has not yet been confirmed. Further insights into the nature of these low mass white dwarfs will be gained by combining the photometric analysis with the results obtained from spectroscopy (see Section 6.4).

For L745-46A (0738-172), GD 95 (0843+358), and Ross 640 (1626+368), we simply assumed for the sake of simplicity a pure helium composition, although these stars are DZA white dwarfs with a small trace of hydrogen on the order of $\mathrm{H} / \mathrm{He} \sim 10^{-4}-10^{-3}$. $\mathrm{H} \alpha$ in these cases is heavily broadened through van der Waals interactions in a helium dominated environment (see Figure 12 of BLR01). These two objects will be analyzed in greater detail in the next section.

Kowalski \& Saumon (2006) suggested that most, if not all, cool DC stars probably have hydrogen-rich atmospheres, based on the analysis of the BRL97 and BLR01 photometry with their improved model atmospheres that include the previously missing red wing opacity from Ly $\alpha$. This does not necessarily imply that all cool white dwarfs have hydrogen atmospheres, however, since cool DZ stars, such as LP 701-29 (2251-070), obviously have helium-dominated atmospheres. We further examine the suggestion of Kowalski \& Saumon below. We have 20 non-DA stars in our sample, most of which appear in Figure 9; the only two stars missing are LP 207-50 (0749+426; ugriz data) and LP 287-39 (2215+368; V magnitude only). Four of these are strong DZ, DQpec, or even DXP stars. Of the remaining 16 objects, 9 are indeed better fitted with pure hydrogen models. Worth mentioning in this category is L40-116 (1315-781), the only non-DA stars in the so-called "non-DA gap" observed in Figure 9 and discussed above. The predicted $\mathrm{H} \alpha$ profile would suggest that this star has a helium-atmosphere, but the spectrum is rather noisy, and we can even catch a glimpse of Zeeman splitting at $\mathrm{H} \alpha$. It is therefore quite possible that L40-116 is a magnetic 
DA white dwarf; improved spectroscopy at higher signal-to-noise ratio would be required for this star.

The remaining 7 objects were originally classified in our analysis as helium-atmosphere white dwarfs. However, a new spectrum of LHS 1421 (0233-242) obtained by Kawka, Vennes, Arazimova, \& Nemeth (2011, in preparation; private communication by S. Vennes) reveals that this star is actually a magnetic DA star with Zeeman splitting observed at $\mathrm{H} \alpha$; we thus decided to adopt instead the hydrogen solution for this object. Also, SCR 0708-6706 (0708-670) has a bad photometric fit, and improved photometry could perhaps change our conclusion about the atmospheric composition of this star. This is also the case for LP 593-56 (0344+014), for which the optical photometry is better fitted with the hydrogen solution while the infrared data favor the helium solution. There is even a hint of $\mathrm{H} \alpha$ in the spectrum. We have thus decided to switch to the hydrogen solution for this object as well. Similarly for LP 287-39 (2215+368), for which we have only a $V$ magnitude in the optical. The pure hydrogen solution could be equally as good, and again, there is even a hint of Zeeman splitting at $\mathrm{H} \alpha$; we thus switched to the hydrogen solution for this star. vB 3 (0743-336) and LHS 378 (1444-174) had ambiguous fits (not shown here) when using the $J H K$ photometry taken from BRL97 and BLR01, but the 2MASS $J H K_{S}$ photometry available for these stars clearly improved our fits using pure hydrogen models. We thus adopted the 2MASS photometry and the pure hydrogen solutions for these 2 objects. Finally, the weak DZ star LP 658-2 (0552-041) is better fitted with a helium-atmosphere model, although the fit is admittedly not perfect; the use of 2MASS photometry did not improve our fit either. However, the sharp calcium lines observed in Figure 7 clearly indicate that this star has a hydrogen atmosphere, otherwise our calculations (not shown here) predict that the calcium line profiles are much shallower and broader than those observed here (contrast LP 658-2 with the other DZ stars displayed in Figure 7). Again, we switched to the hydrogen solution for this object. Based on the previous discussion, we are thus inclined to agree with the conclusions of Kowalski \& Saumon (2006) that most, if not all, cool DC stars probably have hydrogen-rich atmospheres.

\subsection{Photometric Analysis of DQ and DZ Stars}

Even though the photometric fits to the DQ and DZ white dwarfs discussed in the previous section appear reasonable, they do require improved model atmospheres when analyzed with the photometric technique. As discussed above, the presence of heavier elements in helium-rich models provides enough free electrons to affect the atmospheric structure significantly, and thus the predicted energy distributions. 


\subsubsection{DQ Stars}

The photometric technique used to fit the energy distributions of DQ stars is described

in Dufour et al. (2005), and is similar to that described above, with the exception that a third fitting parameter, the carbon abundance, is also taken into account. Spectroscopic observations in the optical (see Figure 51) are used to determine the carbon abundance by fitting the $\mathrm{C}_{2}$ Swan bands at the values of $T_{\text {eff }}$ and $\log g$ obtained from a first fit to the energy distribution with an arbitrary carbon abundance. This improved carbon abundance is then used to obtain new estimates of the atmospheric parameters from the energy distribution, and so forth. This iterative procedure is repeated until $T_{\text {eff }}, \log g$, and the carbon abundance converge to a consistent photometric and spectroscopic solution. Two objects in our sample have carbon features detectable only in the ultraviolet, L97-3 (0806-661) and LDS 678A (1917-077). For these two stars, we replace the optical spectra in our fitting procedure with low-dispersion IUE (International Ultraviolet Explorer) spectra taken from Holberg et al. (2003).

Since our models do not yet include the opacity from the pressure-shifted $\mathrm{C}_{2}$ Swan bands required to properly analyze the peculiar DQ stars (DQpec; Figure 6), we adopt the results based on our previous analysis with pure helium models, with the exception of LHS 290 (1043-188) and G225-68 (1633+572), for which we simply fix the carbon abundance at $\log \mathrm{C} / \mathrm{He}=-7.0$ and -7.5 , respectively. The corresponding model spectra reproduce the depth of the $\mathrm{C}_{2}$ molecular bands fairly well, and the fact that the bands do not appear at the proper wavelengths is less crucial than obtaining the correct amount of free electrons in our models. We were unable to achieve similar qualitative fits for the other peculiar DQ white dwarfs in our sample, and this is why we relied on our previous analysis with pure helium models for these stars. Note that for LHS $2229(1008+290)$, we neglected the $B$ and $V$ photometric measurements, which are affected by the strong carbon features present in this star. A similar problem arises for LP 790-29 (1036-204), for which the strong molecular bands make it extremely difficult to model, even approximately.

The results for the DQ stars in our sample are presented in Figure 12, As before, observed fluxes are represented by error bars, while model fluxes are shown as filled circles. The spectroscopic observations used to constrain the carbon abundance in the fitting procedure are displayed in the right panels. Overall, the photometric energy distributions and the optical or UV spectra are well reproduced by our models for the normal DQ stars, with the exception of BPM 27606 (2154-512), for which the predicted depth of the $\mathrm{C}_{2}$ Swan bands is a bit too shallow, because we give a higher weight to the photometry in our fitting procedure. For the DBQA star LDS 678A (1917-077), we measure a hydrogen abundance of $\log \mathrm{H} / \mathrm{He} \sim-5$ based on our fit (not shown here) to the weak $\mathrm{H} \alpha$ absorption feature (see 
Figures 8 and 11). Since the photometric fits discussed here assume $\mathrm{H} / \mathrm{He}=0$, we used a smaller model grid with $\log \mathrm{H} / \mathrm{He}=-5$ and found atmospheric parameters that are almost identical to those obtained here without hydrogen.

As discussed by Dufour et al. (2005), even though the quality of the photometric fits displayed in Figure 12 have not improved with respect to the results obtained with pure helium models (i.e., Figure 11), the values of $T_{\text {eff }}$ and $\log g$ have been significantly reduced $(\sim 0.3$ dex in $\log g)$ when carbon is properly included in the model atmospheres.

GD 184 (1529+141; NLTT 40489), an extremely rare DAQ white dwarf, was discovered by Kawka \& Vennes (2006) who assigned a temperature of $T_{\text {eff }}=5250 \mathrm{~K}$ based on a spectroscopic fit to the weak hydrogen lines (no carbon feature had been reported, however). Our photometric fit based on pure hydrogen models suggests, on the other hand, a much higher temperature around $T_{\text {eff }} \sim 8900 \mathrm{~K}$, although in this case, the predicted $\mathrm{H} \alpha$ profile is much too strong, as if the absorption line was diluted by a featureless DC companion. Given the simultaneous presence of weak $\mathrm{C}_{2}$ absorption features in the optical spectrum, the simplest explanation is that GD 184 is an unresolved double degenerate system composed of a cool DA star and a much hotter DQ star. This interpretation can be tested further by attempting to fit simultaneously the photometric and spectroscopic measurements with composite models. Given the photometric and spectroscopic constraints as well as the large number of fitting parameters, we simply assume $\log g=8$ for both components of the system, and only allow the temperature and the carbon abundance of the DQ white dwarf to vary. The temperature of the DA star is then varied until a proper match to $\mathrm{H} \alpha$ is reached. Our best photometric and spectroscopic fits are displayed in Figure 13, the contribution of each component of the system is also shown in the top panel. Even though this solution represents an excellent match to the observations (the 2MASS photometry has admittedly large uncertainties), it is obviously not a unique solution, however. A trigonometric parallax measurement could help to further constrain the parameters of both stars. This is the second such DA+DQ system ever identified, the first being NLTT 16249 discovered by Vennes \& Kawka (2012), who also reported the presence of photospehric traces of nitrogen in the spectrum of the DQ component.

\subsubsection{DZ Stars}

The fitting procedure for DZ stars is similar to that described above for DQ stars with the exception that spectroscopic observations of the $\mathrm{Ca}$ II H \& K doublet are used to determine the metal abundance (see also Dufour et al. 2007). For the abundance of other heavier elements, not visible spectroscopically, we assume solar ratios for relative abun- 
dances with respect to calcium. Also, since invisible traces of hydrogen may affect the predicted metallic absorption features (see Dufour et al. 2007 for details), we study the influence of this additional parameter by using model grids calculated with hydrogen abundances of $\log \mathrm{H} / \mathrm{He}=-3,-4$, and -5 . For the three DZA stars in our sample - L745-46A (0738-172), GD 95 (0843+358), and Ross $640(1626+368)$ - the hydrogen abundance is measured directly by fitting H $\alpha$. For vMa $2(0046+051)$, LEHPM 2-220 (1009-184), and G139-13 (1705+030), our fit to the calcium lines could be greatly improved by including hydrogen in our models, and we thus determined indirectly the hydrogen abundance in these stars by fitting the calcium lines with $\mathrm{H} / \mathrm{He}$ as a free parameter; the hydrogen abundance in LEHPM 2-220 was actually forced to $\log \mathrm{H} / \mathrm{He}=-3$ to avoid extrapolation outside of our model grid. Finally, we assumed a value of $\log \mathrm{H} / \mathrm{He}=-5$ for the remaining DZ stars in our sample, with the exception of LP 701-29 (2251-070) for which we used hydrogen-free models to avoid collision-induced absorption by molecular hydrogen in the infrared, which is not observed.

The results for the DZ stars in our sample are presented in Figure 14. The spectroscopic observations used to determine the metal and hydrogen abundances in the fitting procedure are shown in the right panels. The predicted energy distributions for all DZ stars in our sample agree extremely well with the observed photometry. Note that our temperature estimate for LP 701-29, $T_{\text {eff }}=4000 \mathrm{~K}$, is at the limit of our grid, and the observed energy distribution suggests a somewhat lower temperature. We can also notice that the Ca II $\mathrm{H}$ \& K doublet is fairly well reproduced in most DZ stars, but small discrepancies still occur. As before, we assign a higher weight to properly fitting the energy distributions rather than the spectroscopic data.

One problematic DZ star in our sample is LP 726-1 (0840-136). Clearly, our best solution totally fails to reproduce the calcium lines. Both the Ca II H \& K doublet and the Ca I $\lambda 4226$ line are predicted too shallow. The calcium doublet can actually be made deeper by increasing the hydrogen abundance in our models. However, since LP 726-1 is extremely cool, even small amounts of hydrogen creates a significant infrared flux deficiency through collision-induced absorptions, which is not observed. An alternative would be to lower the $\log g$ value. This solution (not shown here) does indeed improve our fit to the Ca II H \& $\mathrm{K}$ doublet, but it still fails to match the Ca I $\lambda 4226$ line. As discussed in Subasavage et al. (2007), it is possible that our failure to simultaneously reproduce the photometric and spectroscopic data is related to additional pressure effects neglected in our equation of state (see also Dufour et al. 2007).

As was the case for DQ stars, the effective temperature and surface gravity are significantly reduced when metals are included in the model atmosphere calculations $(\sim 1000 \mathrm{~K}$ 
in $T_{\text {eff }}$ and $\sim 0.3$ dex in $\log g$ ). For objects with trigonometric parallax measurements, the mean surface gravity is now much closer to the canonical $\log g=8$ value.

\section{Spectroscopic Analysis}

The atmospheric parameters of DA stars with well-defined Balmer lines $\left(T_{\text {eff }} \gtrsim 6500 \mathrm{~K}\right)$ can be determined precisely from the optical spectra using the so-called spectroscopic technique developed by Bergeron et al. (1992a). A similar approach can of course be used for (hot) DB stars, although none have been identified in our nearby sample. The technique relies on detailed fits to the observed normalized Balmer line profiles with model spectra, convolved with the appropriate Gaussian instrumental profile. We use the same Levenberg-Marquardt nonlinear least-squares fitting method described above. In this case the $\chi^{2}$ minimization procedure uses all Balmer lines simultaneously to determine the atmospheric parameters $T_{\text {eff }}$ and $\log g$. In the case of contamination by an unresolved main-sequence companion, usually an M dwarf, we simply exclude from the fit the absorption lines that are contaminated (usually $\mathrm{H} \beta$ ). For two DAZ stars in our sample, G74-7 (0208+396) and G180-63 (1633+433), we rely on the fitting technique and model atmospheres described in Gianninas et al. (2011), which include the opacity from Ca II H \& K known to contaminate the $\mathrm{H} \epsilon$ line at the spectral resolution used here. For GD $362(1729+371)$, we adopt directly the solution of Tremblay et al. (2010) based on mixed hydrogen and helium model atmospheres.

The results of or our spectroscopic fits are displayed in Figure 15] in order of right ascension. In all cases, the model spectra match perfectly the observations, including the two DAZ stars discussed above. There is an obvious contamination at $\mathrm{H} \beta$ in LHS 1660 (0419-487) from the M dwarf companion, and this line has been omitted from our fit. We also note a small discrepancy in the line cores of LP 907-37 (1350-090) due to the presence of a relatively weak $(\sim 100 \mathrm{kG})$ magnetic field (Schmidt \& Smith 1994).

Even though the spectroscopic technique is arguably the most accurate method for measuring the atmospheric parameters of DA stars, it has an important drawback at low effective temperatures $\left(T_{\text {eff }} \lesssim 13,000 \mathrm{~K}\right)$ where spectroscopic values of $\log g$ are significantly larger than those of hotter DA stars. This so-called high-log $g$ problem has been discussed at length in Tremblay et al. (2010) and references therein. The most promising solution for this problem was a mild and systematic contamination of the atmospheric regions by helium brought to the surface by convective mixing, a process that would mimic the high log $q$ values inferred from the spectroscopic technique based on pure hydrogen models (Bergeron et al. 1990). However, this suggestion has been refuted by Tremblay et al. (2010), who convincingly showed, using Keck high-resolution spectra of cool DA stars, that helium is simply not 
present in these stars. More recently, Tremblay et al. (2011b) showed that this high- $\log g$ problem is instead related to the limitations of the mixing-length theory used to describe the convective energy transport in DA stars, and that more realistic, 3D hydrodynamical model atmospheres are required in order to obtain a surface gravity distribution that resembles that of hotter radiative-atmosphere DA stars.

The high- $\log g$ problem is particularly problematic for the study of the white dwarfs in the nearby sample. Indeed, out of the 165 white dwarfs analyzed in this paper, 51 are DA stars in the $6000-13,000 \mathrm{~K}$ temperature range with optical spectra available. Since photometry is available for only 22 of these objects, we must therefore rely heavily on spectroscopic $\log g$ values to estimate distances. Such spectroscopic distances are obtained by combining $V$ magnitudes with absolute visual magnitudes calculated from model atmospheres at the spectroscopic values of $T_{\text {eff }}$ and $\log g$, and more reliable estimates of surface gravities thus become essential. Unfortunately, full 3D model atmosphere grids are not available yet, and we must therefore rely on a more approximate procedure.

In what follows, we attempt to derive an empirical correction based on a statistically large and representative sample of DA white dwarfs. The best characterization of the high$\log g$ problem can be found in the recent study of Tremblay et al. (2011a) who analyzed the DA stars identified in the Data Release 4 of the Sloan Digital Sky Survey. In particular, the $\log g$ distribution of DA stars as a function of effective temperature, shown in their Figure 18 and reproduced here in the top panel of Figure 16, shows a significant increase in the $\log g$ values at low temperatures, with a distinctive triangular shape (see Section 4.2 of Tremblay et al. 2011a for a more elaborate discussion). We next fit a third order polynomial through the SDSS data points with $T_{\text {eff }}<14,000 \mathrm{~K}$ in this $T_{\text {eff }}-\log g$ diagram, using average bins of $500 \mathrm{~K}$ in temperature. The result of this polynomial fit is displayed in Figure 16, and is given by the expression $\log g=1.292+1.862 \times 10^{-3} T_{\text {eff }}-1.598 \times 10^{-7} T_{\text {eff }}^{2}+4.368 \times 10^{-12} T_{\text {eff }}^{3}$, valid between $T_{\text {eff }}=7000 \mathrm{~K}$ and $14,000 \mathrm{~K}$. A low order polynomial was preferred in order to ensure a certain smoothness in our correction procedure, and to get rid of any possible large variations due to the inhomogeneous distribution of stars between consecutive bins. Careful attention was also given to remove all stars outside one standard deviation from the mean $\log g$ value in each bin. By doing so, we want to make sure that we eliminate any possible bias that could result from any excess of high- or low-mass stars in a given bin. Also shown in Figure 16 is a $0.594 M_{\odot}$ evolutionary track, which corresponds to the median mass of DA stars above 13,000 K determined by Tremblay et al. (2011a, see their Table 4). Finally, the empirical correction we apply to our spectroscopic $\log g$ values is simply given by the difference between the black and red curves in Figure 16, at any given temperature.

The spectroscopic $\log g$ values for the DA stars in the SDSS corrected in this fashion are 
displayed as a function of effective temperature in the bottom panel of Figure 16. The continuity of the $\log g$ distribution observed here through the entire temperature range suggests that our correction procedure is reasonably sound. We point out that our $\log g$ correction procedure directly results in larger spectroscopic distances, and as such, the number of stars in our local sample, defined within a given volume of space, might eventually be reduced. From this point on, all spectroscopic $\log g$ values given below take into account the correction discussed above.

\section{Selected Results}

\subsection{Comparison of Fitting Techniques}

We compare in Figure 17 the effective temperature and absolute magnitude for the 23 white dwarfs in our sample, all of the DA type, that have both spectroscopic and photometric measurements. In general, the $T_{\text {eff }}$ and $M_{V}$ values agree well with both techniques, with a few exceptions labeled in the figure and discussed below. We also notice, for the hotter objects, a small but systematic offset of $\sim 300 \mathrm{~K}$ between spectroscopic and photometric temperatures — with $T_{\text {eff }}$ (spec) $>T_{\text {eff }}$ (phot) — which might suggest that the high- $\log g$ problem probably affects the spectroscopic temperatures as well, and that the expected $\log g$ and $T_{\text {eff }}$ corrections are somehow correlated.

Two objects, G1-45 (0101+048) and L587-77A (0326-273), stand out in both panels of Figure 17. As discussed above, these are well known unresolved double degenerate systems, and the discrepancy between spectroscopic and photometric temperatures suggests that both components of the system probably have different effective temperatures or even spectral types - a DA+DC system for instance. This would provide a natural explanation for the discrepant fit observed at H $\alpha$ for L587-77A (see Figure 11). L870-2 (0135-052), labeled in the bottom panel, is also a well known unresolved double degenerate binary. The fact that

it does not stand out in the upper panel is due to the fact that both components of the system have comparable effective temperatures (Bergeron et al. 1989). Two other objects in the bottom panel, LHS 3163 (1609+135) and L24-52 (2105-820), have higher than average $\log g$ values based on the photometric analysis, and there is no indication that these stars are overluminous and thus binaries. Interestingly enough, if we adopt instead the uncorrected spectroscopic $\log g$ values for these two white dwarfs, the spectroscopic and photometric surface gravities agree almost perfectly, suggesting that perhaps the high-log $g$ problem does not affect every cool DA star in the same fashion.

The last object labeled in the bottom panel of Figure 17 is GD 1212 (2236-079), a 
relatively cool $\left(T_{\text {eff }} \sim 11,000 \mathrm{~K}\right) \mathrm{ZZ}$ Ceti white dwarf with a dominant period around $1200 \mathrm{~s}$ (Gianninas et al. 2006), which shows a difference of 0.32 dex in $\log g$ between both fitting

techniques. We have an excellent trigonometric parallax measurement from Subasavage et al. (2009) for this object, so there is no reason to expect our photometric $\log g$ determination to be too far off. The photometric and spectroscopic fits displayed in Figures 11 and 15, respectively, also appear perfectly normal. The only peculiarity is our fit at $\mathrm{H} \alpha$ where the observed line profile is significantly shallower than predicted by our models. Such a discrepancy is usually the signature of the dilution of a DA star by an unresolved DC companion (see the extreme case of $1529+141$ in Figure 11). If this interpretation is correct, the contribution of this featureless companion to the total luminosity of the system could perhaps account for the small $\sim 0.5 \%$ amplitude variations observed in GD 1212 (Gianninas et al. 2006), which are at odds with the general tendency to observe large amplitudes in cooler, long-period ZZ Ceti stars.

\subsection{Adopted Atmospheric Parameters}

Data sets appropriate for a photometric or spectroscopic analysis could not be found for 3 stars in our sample - GJ 86 B (0208-510), HD 27442 B (0415-594), and vB 4 (1132-325) - all three of which are Sirius-like systems. Of the 165 remaining white dwarfs analyzed in this paper, 68 have spectroscopic $T_{\text {eff }}$ and $\log g$ determinations while 118 have photometric determinations, 29 of which have no trigonometric parallax measurements and a value of $\log g=8$ had to be assumed.

The final parameters for all white dwarfs in our sample are selected using the following criteria. For stars with $T_{\text {eff }}>12,000 \mathrm{~K}$, we adopt the spectroscopic solution since these are the only ones available anyway. To circumvent the high-log $g$ problem below $T_{\text {eff }}=13,000 \mathrm{~K}$, we favor the photometric solution over the spectroscopic solution, when both are available, except for stars with no trigonometric parallax measurement, in which case we adopt the spectroscopic solution, if available. As already mentioned above, for GD 362 (1729+371), we substitute the solution of Tremblay et al. (2010) based on mixed hydrogen and helium model atmospheres.

Our final results are presented in Table 2 where we give for each object the effective temperature $\left(T_{\text {eff }}\right)$, surface gravity $(\log g)$, stellar mass $\left(M / M_{\odot}\right)$, atmospheric composition (H- or He-dominated, or mixed $\mathrm{H} / \mathrm{He})$, absolute visual magnitude $\left(M_{V}\right)$, luminosity $\left(L / L_{\odot}\right)$, distance $(D)$, white dwarf cooling time, and the method employed to obtain the atmospheric parameters. For the spectroscopic solutions, we provide here the corrected $\log g$ values, and those differ from the uncorrected values given in Figure 15. Whenever necessary to derive the 
above quantities, we rely on the same evolutionary models as before. For the photometric solutions, the distance given in Table 2 is obtained directly from the trigonometric parallax measurement, if available, or from the fitted solid angle $\pi(R / D)^{2}$ assuming a radius corresponding to $\log g=8$ (the latter are noted with $\sigma_{\log g}=0$ in Table 2). For the spectroscopic solutions, the distance is again obtained directly from the trigonometric parallax measurement, if available, otherwise it is derived from the distance modulus, $V-M_{V}=5 \log D-5$, with the $V$ magnitudes taken from Table 1 and the $M_{V}$ values calculated from model atmospheres at the spectroscopic $T_{\text {eff }}$ and $\log g$ values.

We finally note that the atmospheric parameters for the DA+DQ system GD 184 $(1529+141)$ given in Table 2, and in particular the distance of $D=60.7 \mathrm{pc}$, are obtained under the assumption of a single star. The deconvolved atmospheric parameters are provided in Figure 13, assuming that both components of the system have $\log g=8$ yields a revised distance of $D \sim 73 \mathrm{pc}$, way beyond our 20 pc limit.

\subsection{Distances}

Based on the adopted distances presented in Table 2, we obtain a list of 131 white dwarfs that are within 20 pc, taking into account the uncertainties. We compare in Figure 18 our distance estimates with those from Table 1 of Sion et al. (2009). The largest differences occur for the objects that were not previously included in Table 2 of Holberg et al. (2008), and for which the distances in Sion et al. represent only crude estimates. We also note that 7 white dwarfs lie between 20 and 21 pc in Table 2, some of which rely on the assumption of $\log g=8$ for the photometric method. To be more conservative, we decided to include these objects in our sample as well, for a grand total of 138 nearby white dwarfs.

A more detailed comparison with Sion et al. reveals that 9 white dwarfs have been removed from their sample - GD 5 (0008+424), LP 294-61 (0108+277), G84-26 (0457-004), LP 207-50 (0749+426), G49-33 (0955+247), SDSS 1124+595 (1124+595), LP 276-033 (1653+385), LHS $3254(1655+215)$, and LP 522-46 $(2322+137)$ — while 20 represent new additions L796-10 (0053-117), G1-45 (0101+048), LHS 1247 (0123-262), GD 25 (0213+396), LHS 1442 (0243-026), LHS 1660 (0419-487), LHS 1734 (0503-174), G111-64 (0810+489), SCR 0818-3110 (0816-310), LHS 2022 (0827+328), G47-18 (0856+331), LHS 2229 (1008+290), LHS 2522 (1208+576), PM J13420-3415 (1339-340), LHS 56 (1756+143), LTT 8189 (2039-202), LTT 8190 (2039-682), G261-43 (2126+734), G128-7 (2248+293), and LHS 4019 (2347+292). Most of these additions were already included in the list of possible white dwarfs within 20 
pc from Holberg et al. (2008, see their Table 43 ). Our improved distance estimates confirm their place in the local sample.

We finally mention that even though the 3 white dwarfs without analyzable data (0208-510, 0415-594, and 1132-325) are not included in our analysis below, they are still part of the local sample. From this point on, when we refer to the local sample, we restrict ourselves to the list of 138 objects in Table 2 with distances inside the $\sim 20$ pc region, as define above. All objects excluded from this local sample are marked with an asterisk in Table 2.

\subsection{Mass Distribution}

Since photometric analyses of stars with no trigonometric parallax measurements assume a value of $\log g=8.0$, these are not taken into account in the analysis of the mass distribution discussed here, but will be included in the calculation of the luminosity function presented in the next section. Also, for the moment, all suspected or confirmed double degenerate systems are considered as single objects for reasons discussed below.

The mass distribution as a function of effective temperature for each star in our sample is displayed in Figure 19. Atmospheric compositions and spectral types are indicated with different symbols. In particular, filled and open symbols represent hydrogen- and helium-rich compositions, respectively. Also superposed in this figure are the theoretical isochrones for our $\mathrm{C} / \mathrm{O}$ core evolutionary models with thick hydrogen layers, as well as the corresponding isochrones with the main sequence lifetime added to the white dwarf cooling age (for $\tau \geq 2$ Gyr isochrones only); here we simply assume (Leggett et al. 1998) $t_{\mathrm{MS}}=10\left(M_{\mathrm{MS}} / M_{\odot}\right)^{-2.5}$ Gyr and $M_{\mathrm{MS}} / M_{\odot}=8 \ln \left[\left(M_{\mathrm{WD}} / M_{\odot}\right) / 0.4\right]$. As can be seen from these results, white dwarfs with $M \lesssim 0.48 M_{\odot}$ cannot have $\mathrm{C} / \mathrm{O}$ cores, and yet have been formed from single star evolution within the lifetime of the Galaxy. Some of these low-mass objects must either be unresolved double degenerates, or single white dwarfs with helium cores. In the former case, the stellar masses inferred from these figures are underestimated - especially if the unresolved components have comparable luminosities, and the corresponding cooling ages derived here become meaningless. The second possibility corresponds to single (or binary) helium-core degenerates whose core mass was truncated by Case B mass transfer before helium ignition was reached (Iben \& Tutukov 1987).

\footnotetext{
${ }^{3}$ The entry for $0827+387$ and $1339+259$ in Table 4 of Holberg et al. (2008) are typographical errors and actually correspond to $0827+328$ and $1339-340$, respectively.
} 
The objects displayed in red in Figure 19 are double degenerate binaries that have been confirmed through radial velocity measurements: G1-45 (0101+048; Maxted et al. 2000), L870-2 (0135-052; Saffer et al. 1988), and L587-77A (0326-073; Zuckerman et al. 2003). The low masses inferred for these systems $\left(M \sim 0.25-0.45 M_{\odot}\right)$ are a direct consequence of the fact that we assumed that these are single objects, and thus overestimated their stellar radius by a factor of $2^{1 / 2}$ (see equation 3 ), if both components are identical. Had we assumed two stars instead of one, a simple calculation yields photometric masses in the range $M \sim 0.48-0.76 M_{\odot}$, right in the bulk of average mass white dwarfs. A good example of this calculation is for L870-2 with an extremely low photometric mass of $0.24 M_{\odot}\left(T_{\text {eff }} \sim 7100 \mathrm{~K}\right)$. Assuming two identical DA stars yields instead $0.46 M_{\odot}$ for both components of the system, in excellent agreement with our spectroscopic mass of $0.47 M_{\odot}$ (note that the spectroscopic mass is not affected by the presence of two DA stars if they have comparable atmospheric parameters; see Figure 1 of Liebert et al. 1991). It turns out, indeed, that the two DA components in the L870-2 system are virtually identical (Bergeron et al. 1989). Hence, most, if not all low-mass stars observed in Figure 19 are probably unresolved double degenerates. These include LHS 1243 (0121-429), LHS 1734 (0503-174), L532-81 (0839-327), G187-8 $(2048+263)$, G128-7 (2248+293), and probably vB 11 (2054-050), although the latter is one of the coolest white dwarfs in our sample, and the atmospheric parameters might be uncertain. A simple mass redetermination for these objects, as prescribed above, is not so simple, however. For instance, L532-81, with a photometric mass of $0.44 M_{\odot}\left(T_{\text {eff }} \sim 9100 \mathrm{~K}\right)$, is already in perfect agreement with its spectroscopic mass of $0.42 M_{\odot}$. In this particular case, assuming two DA stars would be the wrong thing to do, as this object appears to be a single DA star. We note, however, that the photometric fit for this object, shown in Figure 11, reveals a small but significant discrepancy at $\mathrm{H} \alpha$, which can easily be explained if the companion star is a DC white dwarf. It is thus not a simple task to deconvolve the individual components of these unresolved binary systems, and their mass determinations should therefore be taken with caution.

Going back to Figure 19, we can see that, not unexpectedly, the local sample is predominantly composed of cool white dwarfs. The first striking feature we observe is the complete absence of white dwarfs with helium-rich atmospheres above $T_{\text {eff }} \sim 12,000 \mathrm{~K}$. To illustrate this more quantitatively, we show in the left panel of Figure 20 the total number of stars as a function of effective temperature per bin size of $2000 \mathrm{~K}$, as well as the individual contribution of the hydrogen-atmosphere white dwarfs. Also, in the right panel, we show the ratio of helium-atmosphere white dwarfs to the total number of stars. The number of helium-rich white dwarfs increases dramatically below 13,000 K, and keeps increasing to a ratio value above $40 \%$ in the range $T_{\text {eff }} \sim 7000-9000 \mathrm{~K}$. Clearly, these results indicate that some physical mechanism is transforming the surface composition of white dwarf stars as 
they cool off. The most obvious mechanism in this temperature range is convective mixing, where the thin convective hydrogen atmosphere is mixed with the deeper and more massive helium convection zone. Since the depth of the hydrogen convection zone increases at lower temperatures, the temperature at which mixing occurs becomes a function of the mass of the hydrogen envelope - the thicker the hydrogen layer, the cooler the mixing temperature. If this interpretation is correct, our results suggest that convective mixing occurs for a significant fraction $(\sim 40 \%)$ of DA stars below $T_{\text {eff }} \sim 10,000 \mathrm{~K}$ or so. This confirms the results obtained by Tremblay \& Bergeron (2007) who performed a model atmosphere analysis of cool white dwarfs with 2MASS infrared photometry available, to show that the ratio of helium- to hydrogen-atmosphere white dwarfs increases gradually from a constant value of $\sim 0.25$ between $T_{\text {eff }}=15,000 \mathrm{~K}$ and $10,000 \mathrm{~K}$ to a value twice as large in the range $10,000>T_{\text {eff }}>8000 \mathrm{~K}$. Our results and those of Tremblay et al. imply that a significant fraction of DA stars, as much as $40 \%$ according to our analysis, may have hydrogen mass layers in the range $M_{\mathrm{H}} / M_{\mathrm{tot}}=10^{-10}$ to $10^{-8}$ (see Tremblay \& Bergeron 2007 for details).

The gradual drop of helium- to hydrogen-atmosphere white dwarfs below $7000 \mathrm{~K}$ in Figure 20] is more difficult to explain. This actually corresponds to the location of the non-DA gap, or deficiency, between roughly $5000 \mathrm{~K}$ and $6000 \mathrm{~K}$ where virtually all white dwarfs are DA stars (see Figure 19). As already discussed above, several exotic mechanisms have been proposed by BRL97 to explain this deficiency, although none appears completely satisfactory, and we simply refer the reader to their discussion (see Figure 35 and Section 6.3.2 of BRL97). Perhaps convective mixing cannot be sustained indefinitely, and the hydrogen and helium layers eventually become well separated again at lower effective temperature.

We also notice in Figure 19 that there appears to be a deficiency of massive stars $(M \gtrsim 1$ $M_{\odot}$ ) below $T_{\text {eff }} \sim 7000 \mathrm{~K}$ with respect to what is observed at higher temperatures. Since these objects have not yet reached the stage of crystallization (which we see here as the rapid reduction of the cooling timescales at high mass), we should expect such cool and massive white dwarfs to be present in the local sample. Another way of saying this, the oldest objects in Figure 19 have a total age of roughly 9.5 Gyr or so, while there are no massive white dwarfs older than 4 Gyr. One could argue that since massive white dwarfs have smaller radii and are thus less luminous, perhaps they have simply gone undetected in proper motion surveys. However, the coolest massive white dwarf in Figure 19 is G108-26 $(0644+025)$, and its luminosity $\left(\log L / L_{\odot}=-3.79\right)$ is not particularly low compared to other objects in Table 2. A more plausible explanation is that all the massive white dwarfs observed here are the results of mergers. In this case their cooling ages cannot be interpreted directly from the isochrones shown here since these are based on single star evolution; the cooling ages inferred here would represent lower estimates at best. If this interpretation is correct, our results suggest that such mergers in the solar neighborhood simply did not have 
enough time to cool off to low temperatures $\left(T_{\text {eff }} \lesssim 7000 \mathrm{~K}\right)$ within the age of the local galactic disk.

A similar situation can be observed at the bottom of the mass distribution in Figure 19, where all low-mass white dwarfs are found only at low effective temperatures, and they all have hydrogen-rich atmospheres. There are no low-mass white dwarfs found at high temperatures, despite the fact these are abundant in spectroscopic analyses of DA stars discovered in UV-excess surveys, such as the PG survey (see, e.g., Figure 12 of Liebert et al. 2005). First, even though the low-mass white dwarfs in Figure 19 are relatively cool, their cooling ages are less than 2 Gyr. As discussed above, most of these objects are probably unresolved double degenerates, and they are likely to be the result of common envelope evolution. As such, it is difficult to interpret their location in this diagram. Also, as discussed in BLR01, we are forced to conclude that this particular evolutionary channel does not produce helium-atmosphere white dwarfs, presumably because the objects that go through this close-binary phase end up with hydrogen layers too massive to allow the DA to DB conversion near $T_{\text {eff }} \sim 30,000 \mathrm{~K}$, or below.

The mass distribution of all white dwarfs in our sample, regardless of their effective temperature, is displayed in Figure 21. We also show the separate contributions of hydrogenand helium-rich stars. The mean mass of the local sample is $\langle M\rangle=0.650 M_{\odot}$ with a standard deviation of $\sigma_{M}=0.161 M_{\odot}$; Holberg et al. (2008) obtained $\langle M\rangle=0.665 M_{\odot}$ for the local white dwarfs in their sample, a value only slightly larger than that reported here. The corresponding mean masses for the hydrogen-atmosphere white dwarfs are $\langle M\rangle=0.647 M_{\odot}$ and $\sigma_{M}=0.171 M_{\odot}$, and for the helium-atmosphere white dwarfs, $\langle M\rangle=0.660 M_{\odot}$ and $\sigma_{M}=0.132 M_{\odot}$. The larger dispersion of the hydrogen stars is a direct result of the presence of both low- and high-mass tails, which are clearly less pronounced in the helium-rich sample. BLR01 found for their hydrogen-rich subsample $\langle M\rangle=0.61 M_{\odot}$, a value noticeably lower than that obtained here. However, a comparison of the mass distributions (see Figure 22 of BLR01) reveals that the discrepancy comes mainly from the low-mass tail, which is far less predominant in our local sample.

The average and median masses for both hydrogen- and helium-atmosphere white dwarfs in Figure 21 are remarkably similar. This result is in sharp contrast with the results obtained by Kepler et al. (2007) in their spectroscopic analyses of DA and DB stars identified in the Data Release 4 of the SDSS. Their results, restricted to effective temperatures above $16,000 \mathrm{~K}$, yield $\langle M\rangle_{\mathrm{DA}} \simeq 0.593 M_{\odot}$ and $\langle M\rangle_{\mathrm{DB}} \simeq 0.683 M_{\odot}$, a significantly larger difference than that obtained here. Since Kepler et al. rely solely on the spectroscopic approach, there could be an indication that this method still needs further investigation. Alternatively, the problem may lie with the analysis of Kepler et al. itself, or with the SDSS data, or both. For 
instance, the mass distribution for brighter DB stars recently obtained by Bergeron et al. (2011), and based also on the spectroscopic technique, yields $\langle M\rangle_{\mathrm{DB}} \simeq 0.671 M_{\odot}$, a somewhat lower value than that obtained from the SDSS data, and only $\sim 0.01 M_{\odot}$ larger than that that quoted above for the local subsample. Similarly, Gianninas et al. (2011) report a mean mass of $\langle M\rangle=0.638 M_{\odot}$ for bright DA stars drawn from the WD Catalog, in good agreement with the value obtained in our analysis. However, the mean mass quoted for the PG magnitude-limited survey, $\langle M\rangle=0.629 M_{\odot}$, is somewhat lower. But this result is actually expected from such magnitude-limited surveys since massive white dwarfs are intrinsically less luminous than their normal mass counterparts, and therefore their number will be significantly underestimated in a survey limited by the magnitude of the star. On the other hand, less massive white dwarfs, with their larger radii and higher luminosities, will be sampled at much larger distances in a magnitude-limited survey, and will thus be overrepresented. Finally, Falcon et al. (2010) measured a mean mass of $0.647 M_{\odot}$ for DA stars based on a gravitational redshift determination of their mean mass, in excellent agreement with our value obtained above for the hydrogen-atmosphere white dwarfs.

Focusing again on the mass distribution of hydrogen-rich white dwarfs displayed in Figure 21, we can see a distinctive high-mass excess near $1 M_{\odot}$, which appears as a very sharp distribution on its own. Clearly, these cannot represent the descendants of single massive progenitors on the main sequence. Indeed, because of the initial-final mass function, the number of massive white dwarfs expected in such a small volume of space is probably close to zero. Hence the massive white dwarfs in our local sample must be again interpreted as mergers in this context. And since we have a volume-limited sample, we are forced to conclude that the fraction of mergers in the Galaxy must be enormous. According to our results, $\sim 6 \%$ of the hydrogen-rich white dwarfs in our sample are mergers. The fact that the mass distribution of these mergers is so narrow also suggests a common evolutionary scenario for these systems. In simple terms, if we interpret these $\sim 1 M_{\odot}$ white dwarfs as the merger of two $\sim 0.5 M_{\odot}$ components, we could even explain the apparent deficiency of hydrogen-rich white dwarfs in the $0.50-0.55 M_{\odot}$ mass range observed in the red histogram shown in Figure 21, which is also apparent from an examination of Figure 19. After all, the local disk is old enough to have formed a sufficiently large number of white dwarfs in this particular mass range, and yet, very few are present in our local sample.

\subsection{Luminosity Function}

The white dwarf luminosity function (WDLF) is, by definition, a measure of the space density of white dwarfs as a function of luminosity, expressed here as the number of stars per 
$\mathrm{pc}^{3}$ per unit of bolometric magnitude. In order to get an accurate picture of this function, the exercise has to be rigorously performed with a well-defined sample, and thus the most important requirement for a proper determination rests on the completeness of the sample. One of the most recent determination of the WDLF, covering the entire range of bolometric magnitudes $\left(7 \lesssim M_{\text {bol }} \lesssim 16\right.$ ) was presented by Harris et al. (2006) using the magnitudelimited Sloan Digital Sky Survey. Several corrections for completeness and contamination had to be made, however, to counterbalance important selection effects present in the SDSS sample. A similar situation arises in the case of all magnitude-limited surveys such as the PG survey (Liebert et al. 2005) or the KUV survey (Limoges \& Bergeron 2010). Using a volume-limited survey avoids most of the problems inherent to these previous determinations, and provides a non-biased sample where completeness issues are better controlled. The most obvious drawback, unfortunately, is the small size of the sample, which may lead to significant statistical uncertainties.

The WDLF calculated here is based on the derived bolometric magnitude of each white dwarf in our sample, obtained via the spectroscopic or photometric results presented in Table 2, with $M_{\mathrm{bol}}=-2.5 \log L / L_{\odot}+M_{\mathrm{bol}}^{\odot}$, where $M_{\mathrm{bol}}^{\odot}=4.75$ is the bolometric magnitude of the Sun. Since a proper account of the number of individual stars in each magnitude bin represents a crucial step in the determination of the WDLF, we consider in our calculation all confirmed and suspected double degenerate binaries, mentioned in Section 6.4, as two individual white dwarfs. We end up this way with a total of 147 individual nearby $(D \lesssim 20$ pc) white dwarfs for the WDLF calculation. Similarly, we deconvolve the individual masses of each system by using the procedure described in Section 6.4, even though we realize this approach is only approximate.

Our results are presented in Figure 22, where the total number of white dwarfs in each bin of bolometric magnitude, divided by the enclosed volume defined by the $20 \mathrm{pc}$ limit, is given as a function of $M_{\text {bol }}$. Also shown at the top is the corresponding effective temperature scale. Our WDLF is also compared with that obtained by Harris et al. (2006) using the SDSS sample, and with that derived by Bergeron et al. (2011) using the DA and DB stars identified in the PG survey (see their Figure 24). The latter is different from the WDLF published by Liebert et al. (2005) since all DA stars in the PG survey have been refitted with the spectroscopic method using models that include the new Stark broadening profiles discussed in Section 3 (Gianninas et al. 2011). Also, both determinations assume a Galactic disk scale height of $250 \mathrm{pc}$.

As discussed by Harris et al. (2006), the space density obtained from the SDSS and the PG surveys agree very well for $7<M_{\text {bol }}<10$ - even better than what is shown in Figure 4 of Harris et al. - but significant deviations occur for fainter magnitude bins where 
the PG survey is believed to be incomplete (Liebert et al. 2005). In contrast, the WDLF obtained from the local sample yields space densities in the same magnitude interval that are larger by a factor of $\sim 2$. This overdensity of white dwarfs above $T_{\text {eff }}=12,000 \mathrm{~K}$ is readily apparent upon a closer examination of the mass distribution shown in Figure 19. We emphasize that all the stars in this temperature range, except one, have distance estimates based on trigonometric parallax measurements, so there is no doubt that these objects indeed belong to the local sample. The only explanation we can come up with to account for this discrepancy is related to the small number of white dwarfs in the brightest magnitude bins. Hopefully, the small number statistics will eventually be improved when the volume of the local sample is increased to larger distances, such as the effort undertaken by Limoges et al. (2010) to define a complete sample of white dwarf stars within a distance of 40 pc from the Sun.

Our results at the faint end of the luminosity function, where the number of stars in each magnitude bin is more statistically significant, are in remarkably good agreement with the SDSS, although the detailed shape is somewhat different. Our result is likely to be more realistic, however, since the fainter magnitude bins in the SDSS are not as well sampled as the brighter bins. For instance, there are only 31 objects in the magnitude bin at the peak of the SDSS distribution, while there are over 500 objects in the magnitude bins near $M_{\mathrm{bol}}=11$.

We cannot help noticing a distinct feature present in all three WDLF calculations displayed in Figure 22, near $M_{\mathrm{bol}}=11$, where the space density decreases slightly before rising again towards lower luminosities. This slight drop in the luminosity function occurs below $T_{\text {eff }} \sim 14,000 \mathrm{~K}$, which corresponds to the temperature range where the atmospheres of DA stars become convective. Perhaps improved treatment of convective energy transport along the lines of the 3D hydrodynamical model atmospheres discussed in Tremblay et al. (2011b) might shift the stars around from one magnitude bin to the other, and make this peculiar feature disappear. This localized feature clearly deserves further investigation.

Finally, by integrating the luminosity function over all magnitude bins, we obtain a measure of the total space density of white dwarfs of $4.39 \times 10^{-3} \mathrm{pc}^{-3}$, and a corresponding mass density of $2.9 \times 10^{-3} M_{\odot} \mathrm{pc}^{-3}$. If we compare these results with the values obtained by Holberg et al. (2008) from the complete portion of the sample within 13 pc - i.e., a space density of $4.8 \pm 0.5 \times 10^{-3} \mathrm{pc}^{-3}$ and a mass density of $3.2 \pm 0.3 \times 10^{-3} M_{\odot} \mathrm{pc}^{-3}$ - we can assess the completeness of our sample to be slightly higher than $90 \%$. Note that our estimate is not affected by the uncertainties in the brighter magnitude bins since the contribution of these stars to the space density is negligible (see Figure 22). 


\section{Conclusion}

A detailed photometric and spectroscopic analysis of 166 nearby white dwarf candidates was presented. Homogeneous determinations of the atmospheric parameters of the local population were performed based on state-of-the-art model atmospheres and improved photometric calibrations. We developed a method for correcting the spectroscopic $\log g$ values at low effective temperatures $\left(T_{\text {eff }}<13,000 \mathrm{~K}\right)$ where the so-called high-log $g$ problem occurs, and which would have prevented us from obtaining reliable mass and distance estimates in this range of temperature.

We determined a mean mass of $\langle M\rangle=0.650 M_{\odot}$ for the complete sample, with corresponding values of $0.647 M_{\odot}$ and $0.660 M_{\odot}$ for the hydrogen- and helium-atmosphere white dwarfs, respectively. There is thus no indication for differences in the mean mass values between these two populations, although the mass distribution of hydrogen white dwarfs contains a significantly larger number of low- and high-mass stars. The mean mass for hydrogen-atmosphere white dwarfs is entirely consistent with that obtained for over 1300 bright DA stars in the WD Catalog, $\langle M\rangle=0.638 M_{\odot}$ (Gianninas et al. 2011). The large fraction of massive stars observed in the local sample has been interpreted as the result of mergers. The main argument for the merger hypothesis is that these massive white dwarfs cannot have evolved from massive main sequence progenitors since the Sun is not located in a region of active star formation where the required massive progenitors could be found.

The local volume-limited sample represents a snapshot of what a representative sample of white dwarf stars might look like. The small spectral atlas displayed here reveals that peculiar white dwarfs are not rare objects, on the contrary. It is thus not too surprising that large surveys such as the SDSS revealed even stranger objects, such as the carbon atmosphere "Hot DQ" stars (Dufour et al. 2008) or white dwarfs with oxygen dominated atmospheres (Gänsicke et al. 2010). Interestingly enough, most of what we know about the mean properties of white dwarf stars comes from the spectroscopic analysis of hot $\left(T_{\text {eff }} \gtrsim\right.$ $15,000 \mathrm{~K})$ DA stars with their comfortable radiative, pure hydrogen atmospheres. Our local sample contains only $\sim 10$ of these DA stars. A more typical white dwarf has instead a convective atmosphere, with quite often a helium-dominated atmosphere, with more than occasional traces of heavier elements. These objects represent a bigger challenge in terms of the modeling of their energy distribution.

The white dwarf luminosity function we derived here for the local sample follows the exact same trend as those previously obtained in many studies, including the SDSS, except at higher luminosities where the local sample contains only a few objects, from a statistical point of view. This portion of the luminosity function will eventually be improved by surveys

aimed at pushing the local sample to a distance of 40 pc (Limoges et al. 2010), an increase 
by a factor of 8 in terms of volume. However, the total space density we derived here, $4.39 \times 10^{-3}$ white dwarfs per cubic parsec, is not affected by this deficiency of hot stars in the local sample. Note that our space density is comparable to the value obtained by Harris et al. (2006) based of the SDSS, $4.6 \times 10^{-3} \mathrm{pc}^{-3}$, but significantly larger than the value derived by Leggett et al. (1998), $3.39 \times 10^{-3} \mathrm{pc}^{-3}$, based on a model atmosphere analysis of 43 white dwarfs identified in the proper motion sample of Liebert et al. (1988).

We wish to deeply thank S. Vennes, A. Kawka, J. P. Subasavage, R.-D. Scholz, S. Salim, B. R. Oppenheimer, S. Lépine, and S. Jordan for sharing their spectroscopic data with us, P.-E. Tremblay, A. Gianninas, and M.-M. Limoges for precious advice and collaboration with some of the data acquisition and reduction, P. Kowalski for sharing with us his calculations of the Ly $\alpha$ opacity, and A. Gianninas for a careful reading of our manuscript. We also wish to thank the director and staff of Steward Observatory and NOAO for the use of their facilities. PD is a CRAQ postdoctoral fellow. This work is supported in part by the NSERC Canada and by the Fund FQRNT (Québec). 


\section{REFERENCES}

Angel, J. R. P., Hintzen, P., \& Landstreet, J. D. 1975, ApJ, 196, L27

Bergeron, P., et al. 2011, ApJ, 737, 28

Bergeron, P., Leggett, S. K., \& Ruiz, M. T. 2001, ApJS, 133, 413 (BLR01)

Bergeron, P., Ruiz, M. T., Hamuy, M., Leggett, S. K., Currie, M. J., Lajoie, C.-P., \& Dufour, P. 2006, ApJ, 625, 838

Bergeron, P., Ruiz, M. T., \& Leggett, S. K. 1992, ApJ, 400, 315

Bergeron, P., Ruiz, M. T., \& Leggett, S. K. 1997, ApJS, 108, 339 (BRL97)

Bergeron, P., Ruiz, M. T., Leggett, S. K., Saumon, D., \& Wesemael, F. 1994, ApJ, 423, 456

Bergeron, P., Saffer, R. A., \& Liebert, J. 1992, ApJ, 394, 228

Bergeron, P., Saumon, D., \& Wesemael, F. 1995a, ApJ, 443, 764

Bergeron, P., Wesemael, F., Fontaine, G., \& Liebert, J. 1990, ApJ, 351, L21

Bergeron, P., Wesemael, F., Lamontagne, R., Fontaine, G., Saffer, R. A., \& Allard, N. F. 1995b, ApJ, 449, 258

Bergeron, P., Wesemael, F., Liebert, J. \& Fontaine, G. 1989, ApJ, 345, L91

Bessell, M. S., \& Brett, J. M. 1988, PASP, 100, 1134

Bessell, M. S., \& Wickramasinghe, D. T. 1978, MNRAS, 182, 275

Bohlin, R. C., \& Gilliland, R. L. 2004, AJ, 127, 3508

Cohen, M. H., Putney, A., \& Goodrich, R. W. 1993, ApJ, 405, L67

Cohen, M., Wheaton, W. A., \& Megeath, S. T. 2003, AJ, 126, 1090

Ducourant, C., Teixeira, R., Hambly, N. C., Oppenheimer, B. R., Hawkins, M. R. S., Rapaport, M., Modolo, J., \& Lecampion, J. F. 2007, A\&A, 470, 387

Dufour, P., Bergeron, P., \& Fontaine, G. 2005, ApJ, 627, 404

Dufour, P., et al. 2007, ApJ, 663, 1291

Dufour, P., Fontaine, G., Liebert, J., Schmidt, G. D., \& Behara, N. 2008, ApJ, 683, 978 
Falcon, R. E., Winget, D. E., Montgomery, M. H., \& Williams, K. A. 2010, ApJ, 712, 585

Farihi, J., Becklin, E. E., \& Zuckerman, B. 2005, ApJS, 161, 394

Farihi, J., Jura, M., \& Zuckerman, B. 2009, ApJ, 694, 805

Fontaine, G., Brassard, P., \& Bergeron, P. 2001, PASP, 113, 409

Gänsicke, B. T., Koester, D., Girven, J., Marsh, T. R., \& Steeghs, D. 2010, Science, 327, 188

Gatewood, G., \& Coban, L. 2009, AJ, 137, 402

Gianninas, A., Bergeron, P., \& Fontaine, G. 2006, AJ, 132, 831

Gianninas, A., Bergeron, P., \& Ruiz, M. T. 2011, ApJ, 743, 138

Gianninas, A., Dufour, P., \& Bergeron, P. 2004, ApJ, 617, L57

Greenstein, J. L. 1976, AJ, 81, 323

Greenstein, J. L. 1984, ApJ, 276, 602

Harris, H. C., et al. 2006, AJ, 131, 571

Hauck, B., \& Mermilliod, M. 1998, A\&AS, 129, 431

Hintzen, P., \& Jensen, E. 1979, PASP, 91, 492

Holberg, J. B., Barstow, M. A., \& Burleigh, M. R. 2003, ApJS, 147, 145

Holberg, J. B., \& Bergeron, P. 2006, ApJ, 132, 1221

Holberg, J. B., Oswalt, T. D., \& Sion, E. M. 2002, ApJ, 571, 512

Holberg, J. B., Sion, E. M., Oswalt, T., McCook, G. P., Foran, S., \& Subasavage, J. P. 2008, AJ, 135, 1225

Hummer, D. G., \& Mihalas, D. 1988, ApJ, 331, 794

Iben, I., Jr., \& Tutukov, A. V. 1987, ApJ, 311, 742

Kawka, A., \& Vennes, S. 2006, ApJ, 643, 402

Kawka, A., Vennes, S., \& Thorstensen, J. R. 2004, AJ, 127, 1702 
Kepler, S. O., Kleinman, S. J., Nitta, A., Koester, D., Castanheira, B. G., Giovannini, O., Costa, A. F. M., \& Althaus, L. 2007, MNRAS, 375, 1315

Kilic, M., et al. 2006, AJ, 131, 582

Kilic, M., Kowalski, P. M., \& von Hippel, T. 2009, AJ, 138, 102

Kilic, M., Thorstensen, J. R., \& Koester, D. 2008, ApJ, 689, L45

Kowalski, P. M. 2010, A\&A, 519, L8

Kowalski, P. M., \& Saumon, D. 2006, ApJ, 651, L137

Leggett, S. K. 1992, ApJS, 82, 351

Leggett, S. K., Ruiz, M. T., \& Bergeron, P. 1998, ApJ, 497, 294

Lépine, S., Rich, R. M., \& Shara, M. M. 2003, AJ, 125, 1598

Lépine, S., Rich, R. M., \& Shara, M. M. 2005, ApJ, 633, L121

Lépine, S., Thorstensen, J. R., Shara, M. M., \& Rich, R. M. 2009, AJ, 137, 4109

Liebert, J., Bergeron, P., \& Holberg, J. B. 2005, ApJS, 156, 47

Liebert, J., Bergeron, P., \& Saffer, R.A. 1991, in 7th European Workshop on White Dwarfs, NATO ASI Series, ed. G. Vauclair \& E. M. Sion (Dordrecht: Kluwer Academic Publishers), 409

Liebert, J., Dahn, C. C., \& Monet, D. G. 1988, ApJ, 332, 891

Limoges, M.-M., \& Bergeron, P. 2010, ApJ, 714, 1037

Limoges, M.-M., Bergeron, P., \& Lépine, S. 2010, American Institute of Physics Conference Series, 1273, 193

Malo, A., Wesemael, F., \& Bergeron, P. 1999, ApJ, 517, 901

Maxted, P. F. L., Marsh, T. R., \& Moran, C. K. J. 2000, MNRAS, 319, 305

McCook, G. P., \& Sion, E. M. 1999, ApJS, 121, 1

Monet, D. et al. 1998, The USNO-A2.0 Catalogue, (U.S. Naval Observatory, Washington $\mathrm{DC})$ 
Oppenheimer, B. R., Hambly, N. C., Digby, A. P., Hodgkin, S. T., \& Saumon, D. 2001, Science, 292, 698

Perryman, M. A. C., et al. 1997, A\&A, 323, L49

Press, W. H., Flannery, B. P., Teukolsky, S. A., \& Vetterling, W. T. 1986, Numerical Recipes (Cambridge: Cambridge University Press)

Provencal, J. L., Shipman, H. L., Wesemael, F., Bergeron, P., Bond, H. E., Liebert, J., \& Sion, E. M. 1997, ApJ, 480, 777

Putney, A. 1997, ApJS, 112, 527

Putney, A., \& Jordan, S. 1995, ApJ, 449, 863

Reimers, D., Jordan, S., Koester, D., Bade, N., Koehler, T., \& Wisotzki, L. 1996, A\&A, 311, 572

Saffer, R. A., Bergeron, P., Koester, D., \& Liebert, J. 1994, ApJ, 432, 351

Saffer, R. A., Liebert, J., \& Olszewski, E. M. 1988, ApJ, 334, 947

Salim, S., \& Gould, A. 2003, ApJ, 582, 1011

Schmidt, G. D., Liebert, J., Harris, H. C., Dahn, C. C., \& Leggett, S. K. 1999, ApJ, 512, 916

Schmidt, G. D., \& Smith, P. S. 1994, ApJ, 423, L63

Scholz, R.-D., Szokoly, G. P., Andersen, M., Ibata, R., \& Irwin, M. J. 2002, ApJ, 565, 539

Sion, E. M., Holberg, J. B., Oswalt, T. D., McCook, G. P., \& Wasatonic, R. 2009, AJ, 138, 1681

Smart, R. L., et al. 2009, A\&A, 404, 317

Subasavage, J. P., Henry, T. J., Bergeron, P., Dufour, P., \& Hambly, N. C. 2008, AJ, 136, 899

Subasavage, J. P., Henry, T. J., Bergeron, P., Dufour, P., Hambly, N. C., \& Beaulieu, T. D. 2007, AJ, 134, 252

Subasavage, J. P., Jao, W.-C., Henry, T. J., Bergeron, P., Dufour, P., Ianna, P. A., Costa, E., \& Méndez, R. A. 2009, AJ, 137, 4547 
Tokunaga, A. T., Simons, D. A, \& Vacca, W. D. 2002, PASP, 114, 180

Tremblay, P.-E., \& Bergeron, P. 2007, ApJ, 657, 10

Tremblay, P.-E., \& Bergeron, P. 2009, ApJ, 696, 1755

Tremblay, P.-E., Bergeron, P., \& Gianninas, A. 2011a, ApJ, 730, 128

Tremblay, P.-E., Bergeron, P., Kalirai, J. S., \& Gianninas, A. 2010, ApJ, 712, 1345

Tremblay, P.-E., Ludwig, H.-G., Steffen, M., Bergeron, P., \& Freytag, B. 2011b, A\&A, 531, L19

van Altena, W. F., Lee, J. T., \& Hoffleit, E. D. 1994, The General Catalogue of Trigonometric Parallaxes (New Haven: Yale Univ. Obs.) (YPC)

Vennes, S., \& Kawka, A. 2003, ApJ, 586, L95

Vennes, S., \& Kawka, A. 2012, ApJ, 745, L12

Vornanen, T., Berdyugina, S. V., Berdyugin, A. V., \& Piirola, V. 2010, ApJ, 720, L52

Wegner, G. 1983, AJ, 88, 109

Wesemael, F., Greenstein, J. L., Liebert, J., Lamontagne, R., Fontaine, G., Bergeron, P., \& Glaspey, J. W. 1993, PASP, 105, 761

Zuckerman, B., Koester, D., Melis, C., Hansen, B. M., \& Jura, M. 2007, ApJ, 671, 872

Zuckerman, B., Koester, D., Reid, I. N., \& Hünsch, M. 2003, ApJ, 596, 477 
Table 1. OBSERVATIONAL DATA

\begin{tabular}{|c|c|c|c|c|c|c|c|c|c|c|c|c|c|}
\hline WD & Name & Spectral Type & $\pi$ (mas) & $\sigma_{\pi}(\mathrm{mas})$ & 1 & 2 & 3 & 4 & Opt. Phot. & $J$ & $H$ & $K$ & Notes \\
\hline $0000-345$ & LHS 1008 & DAH & 75.7 & 9.0 & 15.46 & 15.02 & 14.72 & 14.45 & $B V R I$ & 14.17 & 14.02 & 13.87 & $1,2,3$ \\
\hline $0008+424$ & GD 5 & DA & $\ldots$ & $\ldots$ & $\ldots$ & 15.23 & $\ldots$ & $\ldots$ & $V$ & $\ldots$ & $\ldots$ & $\ldots$ & \\
\hline $0009+501$ & LHS 1038 & DAP & 90.6 & 3.7 & 14.81 & 14.36 & 14.08 & 13.77 & $B V R I$ & 13.41 & 13.26 & 13.21 & $1,2,3$ \\
\hline $0011-134$ & LHS 1044 & DAH & 51.3 & 3.8 & 16.52 & 15.89 & 15.56 & 15.22 & $B V R I$ & 14.85 & 14.62 & 14.52 & $1,2,3$ \\
\hline $0011-721$ & LP 50-73 & DA & $\ldots$ & $\ldots$ & $\cdots$ & 15.17 & 14.87 & 14.55 & $V R I$ & 14.21 & 13.97 & 13.92 & $4,5,6$ \\
\hline $0038-226$ & LHS 1126 & DQpec & 110.54 & 1.18 & 15.20 & 14.50 & 14.08 & 13.71 & $B V R I$ & 13.32 & 13.47 & 13.71 & $1,2,7$ \\
\hline $0046+051$ & vMa 2 & $\mathrm{DZ}$ & 226.95 & 5.35 & 12.91 & 12.39 & 12.13 & 11.90 & $B V R I$ & 11.69 & 11.61 & 11.52 & $8,9,10$ \\
\hline $0053-117$ & L796-10 & DA & $\ldots$ & $\ldots$ & $\ldots$ & 15.26 & $\ldots$ & $\ldots$ & $V$ & $\ldots$ & $\ldots$ & $\ldots$ & \\
\hline $0101+048$ & G1-45 & DA & 46.9 & 3.8 & 14.26 & 14.00 & 13.83 & 13.66 & $B V R I$ & 13.51 & 13.39 & 13.38 & $3,8,9$ \\
\hline $0108+277$ & LP 294-61 & DA & $\ldots$ & $\ldots$ & 16.60 & 16.15 & 15.60 & $\ldots$ & $B V R$ & 15.22 & 15.04 & 14.87 & $5,11,12$ \\
\hline $0115+159$ & LHS 1227 & DQ & 64.9 & 3.0 & 13.95 & 13.85 & 13.74 & 13.65 & $B V R I$ & 13.72 & 13.72 & 13.74 & $1,2,3$ \\
\hline $0121-429$ & LHS 1243 & $\mathrm{DAH}$ & 54.61 & 0.96 & $\ldots$ & 14.83 & 14.52 & 14.19 & $V R I$ & 13.86 & 13.63 & 13.53 & $5,7,13$ \\
\hline $0123-262$ & LHS 1247 & $\mathrm{DC}$ & $\ldots$ & $\ldots$ & 15.39 & 15.06 & 14.84 & 14.62 & $B V R I$ & 14.50 & 14.36 & 14.38 & 1,2 \\
\hline $0135-052$ & L870-2 & DA & 81.0 & 2.8 & 13.19 & 12.86 & 12.63 & 12.38 & $B V R I$ & 12.12 & 11.94 & 11.92 & $1,2,3$ \\
\hline $0141-675$ & LHS 145 & DA & 102.80 & 0.85 & $\ldots$ & 13.82 & $\ldots$ & $\ldots$ & $V$ & $\ldots$ & $\ldots$ & $\ldots$ & 7 \\
\hline $0148+467$ & GD 279 & DA & 63.08 & 3.79 & $\ldots$ & 12.44 & $\ldots$ & $\ldots$ & $V$ & $\ldots$ & $\ldots$ & $\ldots$ & 10 \\
\hline $0148+641$ & G244-36 & DA & $\ldots$ & $\ldots$ & $\ldots$ & 14.00 & $\ldots$ & $\ldots$ & $V$ & $\ldots$ & $\ldots$ & $\ldots$ & \\
\hline $0208+396$ & G74-7 & DAZ & 59.8 & 3.5 & 14.84 & 14.51 & 14.26 & 14.04 & $B V R I$ & 13.80 & 13.65 & 13.63 & $1,2,3$ \\
\hline $0208-510$ & GJ 86 B & DA & 91.63 & 0.61 & $\ldots$ & $\ldots$ & $\ldots$ & $\ldots$ & & $\ldots$ & $\ldots$ & $\ldots$ & $6,10,14$ \\
\hline $0213+396$ & GD 25 & DA & $\ldots$ & $\ldots$ & $\ldots$ & 14.54 & $\ldots$ & $\ldots$ & $V$ & $\ldots$ & $\ldots$ & $\ldots$ & \\
\hline $0213+427$ & LHS 153 & DA & 50.2 & 4.1 & 16.95 & 16.22 & 15.77 & 15.37 & $B V R I$ & 14.98 & 14.73 & 14.52 & $3,8,9$ \\
\hline $0230-144$ & LHS 1415 & DA & 64.0 & 3.9 & 16.46 & 15.77 & 15.34 & 14.93 & $B V R I$ & 14.43 & 14.17 & 14.11 & $1,2,3$ \\
\hline $0233-242$ & LHS 1421 & $\mathrm{DC}$ & $\ldots$ & $\ldots$ & $\ldots$ & 15.94 & 15.43 & 14.93 & $V R I$ & 14.45 & 14.34 & 14.12 & 4,5 \\
\hline $0236+259$ & G36-29 & DA & $\ldots$ & $\ldots$ & 16.60 & 16.29 & 15.80 & $\ldots$ & $B V R$ & 14.91 & 14.61 & 14.47 & $5,11,12$ \\
\hline $0243-026$ & LHS 1442 & DAZ & 47.1 & 5.0 & 15.94 & 15.54 & 15.28 & 15.01 & $B V R I$ & 14.71 & 14.49 & 14.51 & $1,2,3$ \\
\hline $0245+541$ & LHS 1446 & $\mathrm{DAZ}$ & 96.6 & 3.1 & 16.28 & 15.36 & 14.84 & 14.36 & $B V R I$ & 13.89 & 13.66 & 13.60 & $1,3,9$ \\
\hline $0255-705$ & LHS 1474 & $\mathrm{DA}$ & $\ldots$ & $\ldots$ & $\ldots$ & 14.08 & $\ldots$ & $\ldots$ & V & $\ldots$ & $\ldots$ & $\ldots$ & \\
\hline $0310-688$ & LB 3303 & DA & 98.50 & 1.46 & $\ldots$ & 11.40 & $\ldots$ & $\ldots$ & $V$ & $\ldots$ & $\ldots$ & $\ldots$ & 10 \\
\hline $0322-019$ & G77-50 & DAZ & 59.5 & 3.2 & 16.94 & 16.12 & 15.66 & 15.24 & $B V R I$ & 14.63 & 14.37 & 14.28 & $1,2,15$ \\
\hline $0326-273$ & L587-77A & $\mathrm{DA}$ & 57.6 & 13.6 & 14.42 & 14.11 & 13.74 & 13.56 & uvby & 13.27 & 13.12 & 13.08 & $3,9,16$ \\
\hline
\end{tabular}


Table 1-Continued

\begin{tabular}{|c|c|c|c|c|c|c|c|c|c|c|c|c|c|}
\hline WD & Name & Spectral Type & $\pi$ (mas) & $\sigma_{\pi}(\mathrm{mas})$ & 1 & 2 & 3 & 4 & Opt. Phot. & $J$ & $H$ & $K$ & Notes \\
\hline $0341+182$ & Wolf 219 & DQ & 52.6 & 3.0 & 15.52 & 15.19 & 14.91 & 14.65 & $B V R I$ & 14.56 & 14.35 & 14.40 & $1,2,3$ \\
\hline $0344+014$ & LP 593-56 & DC & $\ldots$ & & $\ldots$ & 16.52 & 16.00 & 15.54 & $V R I$ & 15.00 & 14.88 & 14.70 & 5,13 \\
\hline $0357+081$ & LHS 1617 & DA & 56.1 & 3.7 & 16.62 & 15.92 & 15.51 & 15.09 & $B V R I$ & 14.59 & 14.33 & 14.26 & $1,2,3$ \\
\hline $0413-077$ & 40 Eri B & DA & 198.24 & 0.84 & $\ldots$ & 9.50 & $\ldots$ & $\ldots$ & $V$ & $\ldots$ & $\ldots$ & $\ldots$ & 10,14 \\
\hline $0415-594$ & HD 27442 B & DA & 54.84 & 0.5 & $\ldots$ & $\ldots$ & $\ldots$ & $\ldots$ & & $\ldots$ & $\ldots$ & $\ldots$ & $6,10,14$ \\
\hline $0419-487$ & LHS 1660 & $\mathrm{DA}+\mathrm{dM}$ & 49.68 & 1.34 & 14.86 & 14.36 & 13.73 & 12.44 & $B V R I$ & 10.72 & 10.15 & 9.85 & $5,7,17$ \\
\hline $0423+120$ & G83-10 & $\mathrm{DC}$ & 57.6 & 2.5 & 15.90 & 15.42 & 15.11 & 14.80 & $B V R I$ & 14.52 & 14.34 & 14.27 & $1,2,15$ \\
\hline $0426+588$ & Stein 2051B & $\mathrm{DC}$ & 181.36 & 3.67 & 12.73 & 12.43 & 12.17 & 11.86 & $B V R I$ & 11.84 & 11.73 & 11.68 & $8,9,10$ \\
\hline $0433+270$ & G39-27 & DA & 56.02 & 1.21 & 16.48 & 15.81 & 15.40 & 15.01 & $B V R I$ & 14.61 & 14.32 & 14.22 & $1,2,10$ \\
\hline $0435-088$ & L879-14 & DQ & 105.2 & 2.6 & 14.08 & 13.75 & 13.43 & 13.18 & $B V R I$ & 13.00 & 12.85 & 12.79 & $1,2,3$ \\
\hline $0457-004$ & G84-26 & DA & $\ldots$ & $\ldots$ & $\ldots$ & 15.30 & $\ldots$ & $\ldots$ & $V$ & $\ldots$ & $\ldots$ & $\ldots$ & \\
\hline $0503-174$ & LHS 1734 & DAH & 45.6 & 4.0 & 16.73 & 16.01 & 15.57 & 15.11 & $B V R I$ & 14.55 & 14.33 & 14.23 & $1,2,3$ \\
\hline $0532+414$ & GD 69 & DA & $\ldots$ & $\ldots$ & $\ldots$ & 14.75 & $\ldots$ & $\ldots$ & $V$ & $\ldots$ & $\ldots$ & $\ldots$ & \\
\hline $0548-001$ & G99-37 & DQP & 90.3 & 2.8 & 15.08 & 14.56 & 14.22 & 13.95 & $B V R I$ & 13.73 & 13.63 & 13.63 & $1,2,3$ \\
\hline $0552-041$ & LP $658-2$ & $\mathrm{DZ}$ & 155.0 & 2.1 & 15.48 & 14.47 & 13.97 & 13.49 & $B V R I$ & 13.02 & 12.90 & 12.82 & $1,3,9$ \\
\hline $0553+053$ & G99-47 & $\mathrm{DAH}$ & 125.1 & 3.6 & 14.78 & 14.16 & 13.78 & 13.41 & $B V R I$ & 12.96 & 12.77 & 12.66 & $1,2,3$ \\
\hline $0642-166$ & Sirius B & DA & 379.21 & 1.58 & $\ldots$ & 8.39 & $\ldots$ & $\ldots$ & $V$ & $\ldots$ & $\ldots$ & $\ldots$ & 10,14 \\
\hline $0644+025$ & G108-26 & DA & 54.2 & 5.5 & 16.06 & 15.71 & 15.48 & 15.25 & $B V R I$ & 15.00 & 14.85 & 14.93 & $1,3,9$ \\
\hline $0644+375$ & G87-7 & DA & 64.91 & 3.37 & $\ldots$ & 12.08 & $\ldots$ & $\ldots$ & $V$ & $\ldots$ & $\ldots$ & $\ldots$ & 10 \\
\hline $0655-390$ & L454-9 & DA & $\ldots$ & $\ldots$ & $\ldots$ & 15.11 & 14.81 & 14.48 & $V R I$ & 14.15 & 13.88 & 13.89 & $4,5,6$ \\
\hline $0657+320$ & LHS 1889 & DA & 53.5 & 0.9 & 17.64 & 16.62 & 16.09 & 15.61 & $B V R I$ & 14.99 & 14.77 & 14.69 & $1,3,9$ \\
\hline $0659-063$ & LHS 1892 & DA & 81.0 & 24.2 & $\ldots$ & 15.43 & & $\ldots$ & $V$ & $\ldots$ & $\ldots$ & $\ldots$ & 3 \\
\hline $0708-670$ & SCR $0708-6706$ & $\mathrm{DC}$ & $\ldots$ & $\ldots$ & $\ldots$ & 16.22 & 15.72 & 15.21 & $V R I$ & 14.71 & 14.65 & 14.47 & $4,5,6$ \\
\hline $0727+482 \mathrm{~A}$ & G107-70A & DA & 90.0 & 1.0 & 16.24 & 15.26 & 14.73 & 14.24 & $B V R I$ & 13.66 & 13.42 & 13.33 & $3,8,9$ \\
\hline $0727+482 B$ & G107-70B & DA & 90.0 & 1.0 & 16.54 & 15.56 & 15.03 & 14.54 & $B V R I$ & 13.96 & 13.72 & 13.63 & $3,8,9$ \\
\hline $0728+642$ & G234-4 & DAP & $\ldots$ & $\ldots$ & 16.65 & 16.28 & 15.81 & 15.78 & $G V R I$ & 14.81 & 14.52 & 14.39 & 5,18 \\
\hline $0736+053$ & Procyon B & DQZ & 285.93 & 0.88 & 11.19 & 10.94 & 10.79 & 10.67 & $B V R I$ & $\ldots$ & $\ldots$ & $\ldots$ & $10,14,19$ \\
\hline $0738-172$ & L745-46A & DZA & 112.4 & 2.7 & 13.30 & 13.06 & 12.88 & 12.72 & $B V R I$ & 12.65 & 12.61 & 12.52 & $3,8,9$ \\
\hline $0743-336$ & vB 3 & $\mathrm{DC}$ & 65.79 & 0.56 & 17.81 & 16.60 & 15.96 & 15.39 & $B V R I$ & 14.78 & 14.55 & 14.40 & $1,5,10,14$ \\
\hline $0747+073.1$ & LHS 239 & $\mathrm{DC}$ & 54.7 & 0.7 & 18.17 & 16.96 & 16.31 & 15.70 & $B V R I$ & 15.05 & 14.90 & 14.86 & $3,8,9$ \\
\hline
\end{tabular}


Table 1-Continued

\begin{tabular}{|c|c|c|c|c|c|c|c|c|c|c|c|c|c|}
\hline WD & Name & Spectral Type & $\pi$ (mas) & $\sigma_{\pi}$ (mas) & 1 & 2 & 3 & 4 & Opt. Phot. & $J$ & $H$ & $K$ & Notes \\
\hline $0747+073.2$ & LHS 240 & DA & 54.7 & 0.7 & 17.69 & 16.63 & 16.05 & 15.55 & $B V R I$ & 14.96 & 14.73 & 14.72 & $3,8,9$ \\
\hline $0749+426$ & LP 207-50 & $\mathrm{DC}$ & $\ldots$ & $\ldots$ & 18.08 & 17.19 & 16.88 & 16.73 & griz & 15.69 & 15.49 & 15.47 & 20,21 \\
\hline $0751-252$ & SCR $0753-2524$ & DA & 56.54 & 0.95 & $\ldots$ & 16.27 & 15.78 & 15.31 & $V R I$ & 14.75 & 14.47 & 14.30 & $4,5,6,7$ \\
\hline $0752-676$ & BPM 4729 & DA & 141.2 & 8.4 & 14.60 & 13.95 & 13.58 & 13.20 & $B V R I$ & 12.79 & 12.52 & 12.43 & $1,2,3$ \\
\hline $0806-661$ & L97-3 & DQ & 52.17 & 1.67 & 13.74 & 13.71 & 13.64 & 13.60 & $B V R I$ & 13.70 & 13.74 & 13.78 & $1,5,7$ \\
\hline $0810+489$ & G111-64 & $\mathrm{DC}$ & $\ldots$ & $\ldots$ & 15.18 & 14.94 & 14.92 & 14.97 & griz & 14.32 & 14.13 & 14.06 & 5,20 \\
\hline $0816-310$ & SCR $0818-3110$ & DZ & $\ldots$ & $\ldots$ & $\ldots$ & 15.43 & 15.21 & 15.05 & $V R I$ & 14.92 & 14.73 & 14.83 & $4,5,6$ \\
\hline $0821-669$ & SCR 0821-6703 & DA & 93.89 & 1.04 & $\ldots$ & 15.34 & 14.82 & 14.32 & $V R I$ & 13.79 & 13.57 & 13.34 & $5,7,13$ \\
\hline $0827+328$ & LHS 2022 & DA & 44.9 & 3.8 & 16.05 & 15.73 & 15.51 & 15.26 & $B V R I$ & 15.01 & 14.85 & 14.84 & $3,8,9$ \\
\hline $0839-327$ & L532-81 & DA & 112.7 & 9.7 & $\ldots$ & 11.86 & 11.77 & 11.65 & $V R I$ & 11.59 & 11.55 & 11.55 & $3,9,22$ \\
\hline $0840-136$ & LP 726-1 & DZ & $\ldots$ & $\ldots$ & $\ldots$ & 15.72 & 15.36 & 15.02 & $V R I$ & 14.62 & 14.41 & 14.54 & 5,13 \\
\hline $0843+358$ & GD 95 & DZA & $\ldots$ & $\ldots$ & 14.75 & 14.80 & 14.93 & 15.14 & griz & 14.68 & 14.63 & 14.59 & 5,20 \\
\hline $0856+331$ & G47-18 & $\mathrm{DQ}$ & 48.8 & 3.4 & 15.19 & 15.16 & 15.03 & 14.97 & $B V R I$ & 15.12 & 15.09 & 15.11 & $1,2,3$ \\
\hline $0912+536$ & G195-19 & $\mathrm{DCP}$ & 97.3 & 1.9 & 14.19 & 13.84 & 13.64 & 13.51 & $B V R I$ & 13.22 & 13.15 & 13.09 & $2,3,8$ \\
\hline $0939+071$ & GR 431 & NWD & $\ldots$ & $\ldots$ & $\ldots$ & 14.91 & $\ldots$ & $\ldots$ & $V$ & $\ldots$ & $\ldots$ & $\ldots$ & 23 \\
\hline $0946+534$ & G195-42 & DQ & 43.5 & 3.5 & 15.35 & 15.18 & 15.05 & 14.90 & $B V R I$ & 14.90 & 14.87 & 14.88 & $1,2,3$ \\
\hline $0955+247$ & G49-33 & DA & 40.9 & 4.5 & 15.30 & 15.06 & 14.94 & 14.74 & $B V R I$ & 14.66 & 14.59 & 14.65 & $3,8,9$ \\
\hline $1008+290$ & LHS 2229 & DQpecP & 67.47 & 0.47 & 18.21 & 17.51 & $\ldots$ & 15.56 & $B V I$ & 15.01 & 14.71 & 14.55 & 24,25 \\
\hline $1009-184$ & LEHPM 2-220 & $\mathrm{DZ}$ & 54.63 & 0.98 & $\ldots$ & 15.44 & 15.18 & 14.91 & $V R I$ & 14.68 & 14.51 & 14.31 & $5,7,13,14$ \\
\hline $1019+637$ & LP $62-147$ & DA & 61.2 & 3.6 & 15.08 & 14.70 & 14.45 & 14.17 & $B V R I$ & 13.83 & 13.63 & 13.65 & $3,8,9$ \\
\hline $1033+714$ & LHS 285 & $\mathrm{DC}$ & $\ldots$ & $\ldots$ & 17.96 & 16.88 & 16.33 & 15.80 & $B V R I$ & 15.19 & 14.84 & 14.98 & 5,17 \\
\hline $1036-204$ & LP 790-29 & DQpecP & 70.00 & 0.66 & 16.37 & 16.28 & 15.57 & 15.35 & $B V R I$ & 14.70 & 14.28 & 14.09 & $1,2,7$ \\
\hline $1043-188$ & LHS 290 & DQpec & 56.9 & 6.5 & 16.09 & 15.52 & 15.03 & $\ldots$ & $B V R$ & 14.62 & 14.41 & 14.36 & $3,8,9$ \\
\hline $1055-072$ & LHS 2333 & DC & 82.3 & 3.5 & 14.63 & 14.33 & 14.13 & 13.91 & $B V R I$ & 13.81 & 13.71 & 13.69 & $3,8,9$ \\
\hline $1116-470$ & SCR $1118-4721$ & $\mathrm{DC}$ & $\ldots$ & $\ldots$ & $\ldots$ & 15.52 & 15.20 & 14.86 & $V R I$ & 14.45 & 14.37 & 14.35 & $4,5,6$ \\
\hline $1121+216$ & Ross 627 & DA & 74.5 & 2.8 & 14.52 & 14.21 & 14.01 & 13.76 & $B V R I$ & 13.58 & 13.40 & 13.40 & $3,8,9$ \\
\hline $1124+595$ & SDSS & $\mathrm{DA}$ & $\ldots$ & $\ldots$ & $\ldots$ & 15.13 & $\ldots$ & $\ldots$ & V & $\ldots$ & $\ldots$ & $\ldots$ & \\
\hline $1132-325$ & vB 4 & $\mathrm{DC}$ & 104.84 & 0.81 & $\ldots$ & $\ldots$ & $\ldots$ & $\ldots$ & & $\ldots$ & $\ldots$ & $\ldots$ & 10,14 \\
\hline $1134+300$ & GD 140 & DA & 65.28 & 3.61 & $\ldots$ & 12.47 & $\ldots$ & $\ldots$ & $V$ & $\ldots$ & $\ldots$ & $\ldots$ & 10 \\
\hline $1142-645$ & LHS 43 & DQ & 216.40 & 2.11 & 11.66 & 11.49 & 11.33 & 11.20 & $B V R I$ & 11.19 & 11.12 & 11.09 & $8,9,10$ \\
\hline
\end{tabular}


Table 1-Continued

\begin{tabular}{|c|c|c|c|c|c|c|c|c|c|c|c|c|c|}
\hline WD & Name & Spectral Type & $\pi$ (mas) & $\sigma_{\pi}(\mathrm{mas})$ & 1 & 2 & 3 & 4 & Opt. Phot. & $J$ & $H$ & $K$ & Notes \\
\hline $1202-232$ & LP $852-7$ & DAZ & 92.37 & 0.91 & $\ldots$ & 12.80 & $\ldots$ & $\ldots$ & $V$ & $\ldots$ & $\ldots$ & $\ldots$ & 7 \\
\hline $1208+576$ & LHS 2522 & DAZ & 48.9 & 4.6 & 16.35 & 15.78 & 15.41 & 15.04 & $B V R I$ & 14.64 & 14.39 & 14.32 & $3,8,9$ \\
\hline $1223-659$ & L104-2 & $\mathrm{DA}$ & 61.53 & 1.16 & $\ldots$ & 13.97 & $\ldots$ & $\ldots$ & $V$ & $\ldots$ & $\ldots$ & $\ldots$ & 7 \\
\hline $1236-495$ & LTT 4816 & DAV & 61.0 & 9.4 & 13.98 & 13.80 & 13.82 & 13.83 & $B V R I$ & 13.92 & 13.90 & 13.98 & $3,8,9$ \\
\hline $1242-105$ & LP 736-4 & DA & ... & $\ldots$ & $\ldots$ & 14.43 & $\ldots$ & $\ldots$ & $V$ & $\ldots$ & $\ldots$ & $\ldots$ & \\
\hline $1257+037$ & LHS 2661 & DA & 60.3 & 3.8 & 16.50 & 15.84 & 15.46 & 15.08 & $B V R I$ & 14.56 & 14.33 & 14.25 & $1,2,3$ \\
\hline $1309+853$ & G256-7 & DAP & 60.70 & 1.00 & 16.77 & 15.97 & $\ldots$ & $\ldots$ & $B V$ & 14.69 & 14.46 & 14.34 & $5,26,27$ \\
\hline $1310+583$ & PG & $\mathrm{DA}$ & $\ldots$ & $\ldots$ & $\ldots$ & 14.09 & $\ldots$ & $\ldots$ & $V$ & $\ldots$ & $\ldots$ & $\ldots$ & \\
\hline $1310-472$ & ER 8 & $\mathrm{DC}$ & 66.5 & 2.4 & 18.52 & 17.13 & 16.41 & 15.72 & $B V R I$ & 15.21 & 15.11 & 15.03 & $1,2,3$ \\
\hline $1315-781$ & L40-116 & DC: & 52.14 & 0.94 & $\ldots$ & 16.16 & 15.73 & 15.35 & $V R I$ & 14.89 & 14.67 & 14.58 & $5,7,13$ \\
\hline $1327-083$ & Wolf $485 \mathrm{~A}$ & DA & 55.5 & 3.77 & $\ldots$ & 12.33 & $\ldots$ & $\ldots$ & $V$ & $\cdots$ & & $\cdots$ & 10 \\
\hline $1334+039$ & Wolf 489 & DA & 121.4 & 3.4 & 15.58 & 14.63 & 14.12 & 13.62 & $B V R I$ & 13.06 & 12.80 & 12.70 & $1,2,3$ \\
\hline $1339-340$ & PM J13420-3415 & DA & $\ldots$ & $\ldots$ & $\ldots$ & 16.43 & 16.00 & 15.56 & $V R I$ & 15.00 & 14.75 & 14.65 & 5,13 \\
\hline $1344+106$ & LHS 2800 & DAZ & 49.9 & 3.6 & 15.50 & 15.12 & 14.90 & 14.64 & $B V R I$ & 14.38 & 14.20 & 14.19 & $3,8,9$ \\
\hline $1345+238$ & LP $380-5$ & $\mathrm{DA}$ & 82.9 & 2.2 & 16.86 & 15.71 & 15.12 & 14.58 & $B V R I$ & 13.92 & 13.67 & 13.59 & $1,3,9$ \\
\hline $1350-090$ & LP 907-37 & DAP & $\ldots$ & $\ldots$ & $\ldots$ & 14.55 & $\ldots$ & $\ldots$ & $V$ & $\ldots$ & $\ldots$ & $\ldots$ & \\
\hline $1425-811$ & L19-2 & DAV & $\ldots$ & $\ldots$ & $\ldots$ & 13.75 & $\ldots$ & $\ldots$ & $V$ & $\ldots$ & $\ldots$ & $\ldots$ & \\
\hline $1444-174$ & LHS 378 & $\mathrm{DC}$ & 69.0 & 4.0 & 17.47 & 16.44 & 15.95 & 15.43 & $B V R I$ & 14.95 & 14.64 & 14.72 & $1,3,5$ \\
\hline $1529+141$ & GD 184 & $\mathrm{DA}+\mathrm{DQ}$ & $\ldots$ & $\ldots$ & 16.56 & 16.58 & 16.64 & 16.77 & griz & 16.12 & 15.92 & 16.44 & 5,20 \\
\hline $1538+333$ & GD 187 & $\mathrm{DA}$ & $\ldots$ & $\ldots$ & $\ldots$ & 15.03 & $\ldots$ & $\ldots$ & $V$ & $\ldots$ & $\ldots$ & $\ldots$ & \\
\hline $1544-377$ & L481-60 & DA & 65.60 & 0.77 & $\ldots$ & 12.78 & $\ldots$ & $\ldots$ & $V$ & $\ldots$ & $\ldots$ & $\ldots$ & 10,14 \\
\hline $1609+135$ & LHS 3163 & $\mathrm{DA}$ & 54.5 & 4.7 & 15.31 & 15.11 & 15.01 & 14.87 & $B V R I$ & 14.77 & 14.76 & 14.75 & $3,8,9$ \\
\hline $1620-391$ & CD-38 10980 & DA & 78.04 & 2.4 & $\ldots$ & 10.98 & $\ldots$ & $\ldots$ & $V$ & & & $\ldots$ & 10,14 \\
\hline $1626+368$ & Ross 640 & DZA & 62.7 & 2.0 & 14.02 & 13.83 & 13.75 & 13.66 & $B V R I$ & 13.58 & 13.57 & 13.58 & $3,8,9$ \\
\hline $1632+177$ & PG & DAZ & $\ldots$ & $\ldots$ & $\ldots$ & 13.08 & $\ldots$ & $\ldots$ & $V$ & $\ldots$ & $\ldots$ & $\ldots$ & \\
\hline $1633+433$ & G180-63 & DAZ & 66.2 & 3.0 & 15.27 & 14.84 & 14.57 & 14.28 & $B V R I$ & 13.95 & 13.76 & 13.73 & $3,8,9$ \\
\hline $1633+572$ & G225-68 & DQpec & 69.2 & 2.5 & 15.49 & 14.99 & 14.68 & 14.38 & $B V R I$ & 14.03 & 13.97 & 13.99 & $3,8,9$ \\
\hline $1647+591$ & G226-29 & DAV & 91.13 & 2.33 & $\ldots$ & 12.24 & $\ldots$ & $\ldots$ & $V$ & $\ldots$ & $\ldots$ & $\ldots$ & 10 \\
\hline $1653+385$ & LP 276-33 & DAZ & $\ldots$ & $\ldots$ & 16.99 & 16.58 & 16.41 & 16.38 & griz & 15.53 & 15.35 & 15.27 & 5,20 \\
\hline $1655+215$ & LHS 3254 & DA & 43.0 & 3.1 & 14.34 & 14.13 & 14.03 & 13.92 & $B V R I$ & 13.89 & 13.80 & 13.85 & $3,8,9$ \\
\hline
\end{tabular}


Table 1-Continued

\begin{tabular}{|c|c|c|c|c|c|c|c|c|c|c|c|c|c|}
\hline WD & Name & Spectral Type & $\pi$ (mas) & $\sigma_{\pi}(\mathrm{mas})$ & 1 & 2 & 3 & 4 & Opt. Phot. & $J$ & $H$ & $K$ & Notes \\
\hline $1657+321$ & LP $331-27$ & DA & $\ldots$ & $\ldots$ & 17.66 & 17.36 & 17.28 & 17.24 & griz & $\ldots$ & $\ldots$ & $\ldots$ & 20 \\
\hline $1705+030$ & G139-13 & $\mathrm{DZ}$ & 57.0 & 5.4 & 15.63 & 15.20 & 14.96 & 14.74 & $B V R I$ & 14.62 & 14.50 & 14.48 & $3,8,9$ \\
\hline $1729+371$ & GD 362 & DAZB & 19.9 & 1.3 & $\ldots$ & 16.23 & $\ldots$ & . & $V$ & $\ldots$ & $\ldots$ & $\ldots$ & 28 \\
\hline $1748+708$ & G240-72 & DXP & 164.7 & 2.4 & 14.61 & 14.13 & 13.60 & 13.08 & $B V R I$ & 12.77 & 12.70 & 12.50 & $2,3,8$ \\
\hline $1756+143$ & LSR $1758+1417$ & DA & $\ldots$ & $\ldots$ & $\ldots$ & 16.30 & 16.12 & 15.69 & $V R I$ & 14.93 & 14.66 & 14.66 & 5,13 \\
\hline $1756+827$ & LHS 56 & DA & 63.9 & 2.9 & 14.69 & 14.34 & 14.12 & 13.87 & $B V R I$ & 13.63 & 13.47 & 13.43 & $3,5,8$ \\
\hline $1814+134$ & LSR $1817+1328$ & DA & 70.3 & 1.2 & $\ldots$ & 15.85 & 15.34 & 14.86 & $V R I$ & 14.38 & 14.10 & 14.08 & $5,13,29$ \\
\hline $1820+609$ & G227-28 & DA & 78.2 & 4.1 & 16.67 & 15.69 & 15.15 & 14.64 & $B V R I$ & 14.07 & 13.81 & 13.80 & $3,5,8$ \\
\hline $1829+547$ & G227-35 & DXP & 66.8 & 5.6 & 16.06 & 15.57 & 15.28 & 14.97 & $B V R I$ & 14.76 & 14.61 & 14.50 & $3,8,9$ \\
\hline $1900+705$ & GW+70 8247 & DAP & 77.0 & 2.3 & 13.31 & 13.25 & 13.24 & 13.23 & $B V R I$ & 13.33 & 13.44 & 13.42 & $3,5,8$ \\
\hline $1917+386$ & G125-3 & DC & 85.5 & 3.4 & 15.06 & 14.61 & 14.31 & 14.04 & $B V R I$ & 13.77 & 13.69 & 13.59 & $2,3,8$ \\
\hline $1917-077$ & LDS 678A & DBQA & 99.2 & 2.5 & 12.58 & 12.54 & 12.41 & 12.30 & $u v b y$ & 12.35 & 12.36 & 12.42 & $3,5,30$ \\
\hline $1919+145$ & GD 219 & DA & 50.5 & 5.5 & 13.51 & 13.44 & 13.06 & 13.05 & uvby & 13.26 & 13.45 & 13.55 & $3,5,30$ \\
\hline $1935+276$ & G185-32 & DAV & 55.7 & 2.9 & $\ldots$ & 12.98 & $\ldots$ & $\ldots$ & $V$ & $\ldots$ & $\ldots$ & $\ldots$ & 3 \\
\hline $1953-011$ & LHS 3501 & $\mathrm{DAH}$ & 87.8 & 2.9 & 13.97 & 13.69 & 13.50 & 13.31 & $B V R I$ & 13.12 & 13.02 & 13.02 & $3,8,9$ \\
\hline $2002-110$ & LHS 483 & DC & 57.7 & 0.8 & 18.11 & 16.95 & 16.36 & 15.86 & $B V R I$ & 15.32 & 15.11 & 15.09 & $1,3,9$ \\
\hline $2007-303$ & L565-18 & DA & 65.06 & 3.85 & $\ldots$ & 12.18 & $\ldots$ & $\ldots$ & $V$ & $\ldots$ & $\ldots$ & $\ldots$ & 10 \\
\hline $2008-600$ & SCR $2012-5956$ & $\mathrm{DC}$ & 60.42 & 0.86 & $\ldots$ & 15.84 & 15.40 & 14.99 & $V R I$ & 14.93 & 15.23 & 15.41 & $5,7,13$ \\
\hline $2032+248$ & G186-31 & DA & 67.65 & 2.32 & $\ldots$ & 11.52 & $\ldots$ & $\ldots$ & $V$ & $\ldots$ & $\ldots$ & $\ldots$ & 10 \\
\hline $2039-202$ & LTT 8189 & DA & 47.39 & 4.04 & $\ldots$ & 12.33 & $\ldots$ & $\ldots$ & $V$ & $\ldots$ & $\ldots$ & $\ldots$ & 10 \\
\hline $2039-682$ & LTT 8190 & DA & $\ldots$ & $\ldots$ & $\ldots$ & 13.45 & $\ldots$ & $\ldots$ & $V$ & $\ldots$ & $\ldots$ & $\ldots$ & \\
\hline $2040-392$ & L495-82 & DA & 44.18 & 0.97 & $\ldots$ & 13.74 & $\ldots$ & $\ldots$ & $V$ & $\ldots$ & $\ldots$ & $\ldots$ & 7 \\
\hline $2047+372$ & G210-36 & DA & 57.87 & 0.69 & $\ldots$ & 12.93 & & $\ldots$ & $V$ & $\ldots$ & $\ldots$ & $\ldots$ & 27 \\
\hline $2048+263$ & G187-8 & DA & 49.8 & 3.4 & 16.55 & 15.63 & 15.11 & 14.63 & $B V R I$ & 14.12 & 13.83 & 13.79 & $1,2,3$ \\
\hline $2048-250$ & LP $872-20$ & DA & $\ldots$ & $\ldots$ & 15.80 & 15.42 & 15.20 & $\ldots$ & $B V R$ & 14.90 & 14.70 & 14.60 & $5,11,12$ \\
\hline $2054-050$ & vB 11 & DC & 56.56 & 4.03 & 17.89 & 16.69 & 16.03 & 15.37 & $B V R I$ & 14.82 & 14.61 & 14.54 & $1,2,10$ \\
\hline $2105-820$ & L24-52 & DAZH & 58.6 & 8.8 & 13.82 & 13.61 & 13.56 & 13.47 & $B V R I$ & 13.52 & 13.53 & 13.58 & $3,8,9$ \\
\hline $2115-560$ & BPM 27273 & DAZ & $\ldots$ & $\ldots$ & $\ldots$ & 14.28 & $\ldots$ & $\ldots$ & $V$ & $\ldots$ & $\ldots$ & $\ldots$ & \\
\hline $2117+539$ & G231-40 & $\mathrm{DA}$ & 50.7 & 7.4 & $\ldots$ & 12.33 & $\ldots$ & $\ldots$ & $V$ & $\ldots$ & $\ldots$ & $\ldots$ & 3 \\
\hline $2126+734$ & G261-43 & DA & 47.1 & 2.4 & $\ldots$ & 12.82 & $\ldots$ & $\ldots$ & $V$ & $\ldots$ & $\ldots$ & $\ldots$ & 3 \\
\hline
\end{tabular}


Table 1-Continued

\begin{tabular}{|c|c|c|c|c|c|c|c|c|c|c|c|c|c|}
\hline WD & Name & Spectral Type & $\pi$ (mas) & $\sigma_{\pi}(\mathrm{mas})$ & 1 & 2 & 3 & 4 & Opt. Phot. & $J$ & $H$ & $K$ & Notes \\
\hline $2138-332$ & L570-26 & $\mathrm{DZ}$ & 64.00 & 1.41 & $\ldots$ & 14.47 & 14.30 & 14.16 & $V R I$ & 14.17 & 14.08 & 13.95 & $5,7,13$ \\
\hline $2140+207$ & LHS 3703 & DQ & 79.9 & 3.2 & 13.37 & 13.24 & 13.10 & 12.98 & $B V R I$ & 12.95 & 12.93 & 12.95 & $3,8,9$ \\
\hline $2151-015$ & G93-53 & $\mathrm{DA}$ & $\ldots$ & $\ldots$ & $\ldots$ & 14.41 & $\ldots$ & $\ldots$ & $V$ & $\ldots$ & $\ldots$ & $\ldots$ & \\
\hline $2154-512$ & BPM 27606 & DQP & 61.63 & 2.67 & 14.93 & 14.74 & 14.30 & 14.13 & $B V R I$ & 14.01 & 13.91 & 13.81 & $1,5,10$ \\
\hline $2159-754$ & LHS 3752 & $\mathrm{DA}$ & $\ldots$ & $\ldots$ & $\ldots$ & 15.06 & $\ldots$ & $\ldots$ & $V$ & $\ldots$ & $\ldots$ & $\ldots$ & \\
\hline $2211-392$ & LEHPM 1-4466 & DA & 53.5 & 2.6 & 16.41 & 15.92 & 15.59 & 15.26 & $B V R I$ & 14.89 & 14.64 & 14.56 & $5,31,32$ \\
\hline $2215+368$ & LP 287-39 & $\mathrm{DC}$ & $\ldots$ & $\ldots$ & $\ldots$ & 16.99 & $\ldots$ & $\ldots$ & $V$ & 15.41 & 15.20 & 14.97 & 5,11 \\
\hline $2226-754$ & SSSPM J2231-7514 & $\mathrm{DC}$ & $\ldots$ & $\ldots$ & $\ldots$ & 16.57 & 15.93 & 15.33 & $V R I$ & 14.66 & 14.66 & 14.44 & 5,13 \\
\hline $2226-755$ & SSSPM J2231-7515 & $\mathrm{DC}$ & $\ldots$ & $\ldots$ & $\ldots$ & 16.88 & 16.17 & 15.51 & $V R I$ & 14.86 & 14.83 & 14.72 & 5,13 \\
\hline $2246+223$ & G67-23 & $\mathrm{DA}$ & 52.5 & 4.1 & 14.56 & 14.39 & 14.34 & 14.25 & $B V R I$ & 14.28 & 14.31 & 14.37 & $3,8,9$ \\
\hline $2248+293$ & G128-7 & DA & 47.8 & 4.2 & 16.20 & 15.54 & 15.14 & 14.75 & $B V R I$ & 14.24 & 14.01 & 13.94 & $1,3,9$ \\
\hline $2251-070$ & LP 701-29 & $\mathrm{DZ}$ & 123.7 & 4.3 & 17.55 & 15.71 & 15.10 & 14.56 & $B V R I$ & 13.86 & 13.63 & 13.47 & $1,2,3$ \\
\hline $2322+137$ & LP 522-46 & DA & 44.9 & 2.0 & 16.47 & 15.86 & 15.57 & 15.47 & griz & 14.53 & 14.34 & 14.21 & $20,21,29$ \\
\hline $2326+049$ & G29-38 & DAZ & 73.4 & 4.0 & $\ldots$ & 13.05 & $\ldots$ & $\ldots$ & $V$ & $\ldots$ & $\ldots$ & $\ldots$ & 3 \\
\hline $2336-079$ & GD 1212 & DAV & 62.72 & 1.70 & $\ldots$ & 13.28 & 13.27 & 13.24 & $V R I$ & 13.34 & 13.34 & 13.35 & $4,5,7$ \\
\hline $2341+322$ & G130-5 & DA & 56.8 & 1.8 & & 12.92 & $\ldots$ & $\ldots$ & $V$ & $\ldots$ & $\ldots$ & $\ldots$ & 3 \\
\hline $2347+292$ & LHS 4019 & DA & 46.5 & 4.1 & 16.35 & 15.76 & 15.41 & 15.04 & $B V R I$ & 14.59 & 14.35 & 14.24 & $1,2,3$ \\
\hline $2351-335$ & LHS 4040 & DA & 42.82 & 2.40 & $\ldots$ & 14.42 & $\ldots$ & $\ldots$ & $V$ & $\ldots$ & $\ldots$ & $\ldots$ & 7 \\
\hline $2359-434$ & LHS 1005 & DA & 127.4 & 6.8 & $\ldots$ & 13.05 & $\ldots$ & $\ldots$ & $V$ & $\ldots$ & $\ldots$ & $\ldots$ & 3 \\
\hline
\end{tabular}

Note. - (1) BVRI from Bergeron. Ruiz. \& Leggett 1997); (2) JHK from Bergeron, Ruiz. \& Leggett (1997); (3) $\pi$ from YPC; (4) VRI from Subasavage et al. (2008); (5) $J H K_{S}$ from 2MASS; (6) Not in Villanova White Dwarf Catalog; (7) $\pi$ from Subasavage et al. (2009); (8) BVRI from Bergeron. Leggett. \& Ruiz (2001); (9) $J H K$ from Bergeron. Leggett. \& Ruiz (2001); (10) $\pi$ from Perrvman et al. (1997); (11) $V$ from Salim \& Gould (2003); (12) BR from Monet et al. (1998); (13) $V R I$ from Subasavage et al. (2007); (14) Sirius-like star; (15) $\pi$ from Smart et al. (2009); (16) uvby from Bessell \& Wickramasinohe (1978); (17) Unpublished BV RI from Bergeron, Ruiz, \& Leggett (1997); (18) BGVRI from Greenstein (1984); (19) BVRI from Provencal et al. (1997); (20) ugriz from SDSS; (21) JHK from Kilic et al. (2009); (22) VRI from Subasavage et al. (2009); (23) Classified DC7 in the PG catalog; (24) BVIJHK from Schmidt et al. (1999); (25) $\pi$ from H. C. Harris. (2010, private communication); (26) $B V$ from Hintzen \& Jensen (1979); (27) $\pi$ from Gatewood \& Coban (2009); (28) $\pi$ from Kilic et al. (2008); (29) $\pi$ from Lépine et al. (2009); (30) uvby from Wegner (1983); (31) BVRI from Bergeron et al. (2005); (32) $\pi$ from Ducourant et al. (2007). 
Table 2. ADOPTED ATMOSPHERIC PARAMETERS OF NEARBY CANDIDATES

\begin{tabular}{|c|c|c|c|c|c|c|c|c|c|}
\hline WD & $T_{\text {eff }}(\mathrm{K})$ & $\log g$ & $M / M_{\odot}$ & Comp. & $M_{V}$ & $\log L / L \odot$ & $D(\mathrm{pc})$ & Age (Gyr) & Notes \\
\hline $0000-345$ & 6643 (149) & $8.45(0.16)$ & $0.88(0.10)$ & $\mathrm{H}$ & 14.41 & -3.82 & $13.2(1.6)$ & 4.07 & 2 \\
\hline $0008+424$ & $7202(107)$ & $8.07(0.07)$ & $0.64(0.04)$ & $\mathrm{H}$ & 13.58 & -3.45 & $21.4(1.1)$ & 1.56 & 1 \\
\hline $0009+501$ & $6502(143)$ & $8.23(0.05)$ & $0.73(0.04)$ & $\mathrm{H}$ & 14.15 & -3.72 & $11.0(0.5)$ & 3.02 & 2 \\
\hline $0011-134$ & $5992(114)$ & $8.21(0.11)$ & $0.72(0.07)$ & $\mathrm{H}$ & 14.44 & -3.85 & $19.5(1.5)$ & 3.73 & 2 \\
\hline $0011-721$ & $6325(203)$ & $8.00(0.00)$ & $0.59(0.00)$ & $\mathrm{H}$ & 13.94 & -3.63 & $17.6(0.7)$ & 1.96 & 2 \\
\hline $0038-226$ & $5229(76)$ & $7.93(0.02)$ & $0.53(0.01)$ & $\mathrm{H}+\mathrm{He}$ & 14.72 & -3.94 & $9.05(0.10)$ & 4.44 & 2 \\
\hline $0046+051$ & $6215(183)$ & $8.16(0.03)$ & $0.68(0.02)$ & $\mathrm{He}$ & 14.17 & -3.77 & $4.41(0.10)$ & 3.30 & 2 \\
\hline $0053-117$ & 7145 (108) & $8.12(0.08)$ & $0.67(0.05)$ & $\mathrm{H}$ & 13.68 & -3.49 & $20.7(1.3)$ & 1.70 & 1 \\
\hline $0101+048$ & $8062(200)$ & $7.54(0.14)$ & $0.36(0.05)$ & $\mathrm{H}$ & 12.36 & -2.96 & $21.3(1.7)$ & 0.63 & 2 \\
\hline $0108+277^{*}$ & 6428 (190) & $8.00(0.00)$ & $0.59(0.00)$ & $\mathrm{H}$ & 13.92 & -3.60 & $28.0(1.5)$ & 1.88 & 2 \\
\hline $0115+159$ & 9119 (319) & $8.17(0.07)$ & $0.69(0.04)$ & $\mathrm{He}$ & 12.91 & -3.10 & $15.4(0.7)$ & 1.04 & 2 \\
\hline $0121-429$ & 6299 (191) & $7.66(0.03)$ & $0.41(0.01)$ & $\mathrm{H}$ & 13.52 & -3.46 & $18.3(0.3)$ & 1.27 & 2 \\
\hline $0123-262$ & 7288 (192) & $8.00(0.00)$ & $0.58(0.00)$ & $\mathrm{He}$ & 13.37 & -3.40 & $21.7(0.8)$ & 1.49 & 2 \\
\hline $0135-052$ & 7118 (165) & $7.18(0.06)$ & $0.24(0.01)$ & $\mathrm{H}$ & 12.40 & -3.00 & $12.3(0.4)$ & 0.66 & 2 \\
\hline $0141-675$ & 6248 ( 99) & $7.81(0.10)$ & $0.48(0.06)$ & $\mathrm{H}$ & 13.82 & -3.55 & $9.73(0.67)$ & 1.56 & 1 \\
\hline $0148+467$ & $14,005(277)$ & $8.04(0.04)$ & $0.63(0.03)$ & $\mathrm{H}$ & 11.45 & -2.26 & $15.9(0.6)$ & 0.26 & 1 \\
\hline $0148+641$ & 9016 (129) & $8.11(0.05)$ & $0.66(0.03)$ & $\mathrm{H}$ & 12.74 & -3.08 & $17.8(0.7)$ & 0.93 & 1 \\
\hline $0208+396$ & $7264(176)$ & $8.00(0.09)$ & $0.59(0.05)$ & $\mathrm{H}$ & 13.39 & -3.39 & $16.7(1.0)$ & 1.39 & 2 \\
\hline $0213+396$ & $9374(135)$ & $8.32(0.05)$ & $0.80(0.03)$ & $\mathrm{H}$ & 12.94 & -3.14 & $20.9(0.9)$ & 1.17 & 1 \\
\hline $0213+427$ & 5507 (108) & $8.10(0.13)$ & $0.64(0.08)$ & $\mathrm{H}$ & 14.72 & -3.93 & $19.9(1.6)$ & 4.02 & 2 \\
\hline $0230-144$ & $5477(93)$ & $8.13(0.09)$ & $0.66(0.06)$ & $\mathrm{H}$ & 14.80 & -3.96 & $15.6(1.0)$ & 4.51 & 2 \\
\hline $0233-242$ & $5312(146)$ & $8.00(0.00)$ & $0.58(0.00)$ & $\mathrm{H}$ & 14.83 & -3.94 & $16.7(0.7)$ & 4.06 & 2 \\
\hline $0236+259^{*}$ & $5666(114)$ & $8.00(0.00)$ & $0.59(0.00)$ & $\mathrm{H}$ & 14.60 & -3.83 & $21.8(0.8)$ & 2.73 & 2 \\
\hline $0243-026$ & $6770(156)$ & $8.18(0.16)$ & $0.70(0.10)$ & $\mathrm{H}$ & 13.90 & -3.62 & $21.2(2.3)$ & 2.31 & 2 \\
\hline $0245+541$ & $5139(82)$ & $8.23(0.05)$ & $0.73(0.03)$ & $\mathrm{H}$ & 15.29 & -4.13 & $10.3(0.3)$ & 7.24 & 2 \\
\hline $0255-705^{*}$ & $10,769(159)$ & $7.95(0.05)$ & $0.57(0.03)$ & $\mathrm{H}$ & 11.86 & -2.67 & $27.8(1.1)$ & 0.47 & 1 \\
\hline $0310-688$ & $16,865(244)$ & $8.10(0.04)$ & $0.67(0.03)$ & $\mathrm{H}$ & 11.21 & -1.97 & $10.2(0.4)$ & 0.16 & 1 \\
\hline $0322-019$ & $5195(87)$ & $8.08(0.08)$ & $0.63(0.05)$ & $\mathrm{H}$ & 14.99 & -4.02 & $16.8(0.9)$ & 5.60 & 2 \\
\hline $0326-273$ & $8483(200)$ & $7.72(0.39)$ & $0.45(0.18)$ & $\mathrm{H}$ & 12.36 & -2.97 & $17.4(4.3)$ & 0.66 & 2 \\
\hline $0341+182$ & 6568 (136) & $7.99(0.09)$ & $0.57(0.06)$ & $\mathrm{He}$ & 13.79 & -3.57 & $19.0(1.1)$ & 1.89 & 2 \\
\hline $0344+014$ & $5170(171)$ & $8.00(0.00)$ & $0.58(0.00)$ & $\mathrm{H}$ & 14.95 & -3.99 & $20.6(1.2)$ & 4.83 & 2 \\
\hline $0357+081$ & 5478 ( 93) & $8.04(0.11)$ & $0.61(0.06)$ & $\mathrm{H}$ & 14.66 & -3.91 & $17.8(1.2)$ & 3.58 & 2 \\
\hline $0413-077$ & $17,100(256)$ & $7.95(0.04)$ & $0.59(0.03)$ & $\mathrm{H}$ & 10.97 & -1.85 & $5.04(0.19)$ & 0.12 & 1 \\
\hline $0419-487$ & $6444(112)$ & $7.12(0.19)$ & $0.22(0.05)$ & $\mathrm{H}$ & 12.80 & -3.14 & $20.1(2.2)$ & 0.81 & 1 \\
\hline $0423+120$ & 6167 (128) & $8.12(0.06)$ & $0.65(0.04)$ & $\mathrm{He}$ & 14.22 & -3.75 & $17.4(0.8)$ & 3.06 & 2 \\
\hline
\end{tabular}


Table 2-Continued

\begin{tabular}{|c|c|c|c|c|c|c|c|c|c|}
\hline WD & $T_{\text {eff }}(\mathrm{K})$ & $\log g$ & $M / M_{\odot}$ & Comp. & $M_{V}$ & $\log L / L_{\odot}$ & $D(\mathrm{pc})$ & Age (Gyr) & Notes \\
\hline $0426+588$ & $7178(182)$ & $8.18(0.02)$ & $0.69(0.02)$ & $\mathrm{He}$ & 13.72 & -3.52 & $5.51(0.11)$ & 2.02 & 2 \\
\hline $0433+270$ & $5629(98)$ & $8.06(0.04)$ & $0.62(0.02)$ & $\mathrm{H}$ & 14.55 & -3.87 & $17.9(0.4)$ & 3.21 & 2 \\
\hline $0435-088$ & $6367(117)$ & $7.93(0.04)$ & $0.53(0.02)$ & $\mathrm{He}$ & 13.86 & -3.59 & $9.51(0.24)$ & 1.85 & 2 \\
\hline $0457-004^{*}$ & $11,408(168)$ & $8.74(0.05)$ & $1.07(0.03)$ & $\mathrm{H}$ & 13.01 & -3.09 & $28.7(1.4)$ & 1.61 & 1 \\
\hline $0503-174$ & $5316(88)$ & $7.62(0.15)$ & $0.38(0.07)$ & $\mathrm{H}$ & 14.30 & -3.75 & $21.9(1.9)$ & 1.90 & 2 \\
\hline $0532+414^{*}$ & $7753(112)$ & $7.87(0.06)$ & $0.52(0.03)$ & $\mathrm{H}$ & 13.00 & -3.20 & $22.4(1.0)$ & 0.97 & 1 \\
\hline $0548-001$ & $6132(104)$ & $8.18(0.04)$ & $0.69(0.03)$ & $\mathrm{He}$ & 14.34 & -3.80 & $11.1(0.3)$ & 3.63 & 2 \\
\hline $0552-041$ & $5182(81)$ & $8.37(0.02)$ & $0.82(0.01)$ & $\mathrm{H}$ & 15.42 & -4.21 & $6.45(0.08)$ & 7.89 & 2 \\
\hline $0553+053$ & $5785(105)$ & $8.22(0.04)$ & $0.72(0.03)$ & $\mathrm{H}$ & 14.65 & -3.91 & $7.99(0.23)$ & 4.25 & 2 \\
\hline $0642-166$ & $25,967(378)$ & $8.57(0.04)$ & $0.98(0.03)$ & $\mathrm{H}$ & 11.22 & -1.53 & $2.64(0.12)$ & 0.10 & 1 \\
\hline $0644+025$ & $7334(182)$ & $8.65(0.12)$ & $1.01(0.07)$ & $\mathrm{H}$ & 14.38 & -3.79 & $18.4(1.9)$ & 3.84 & 2 \\
\hline $0644+375$ & $22,288(341)$ & $8.10(0.05)$ & $0.69(0.03)$ & $\mathrm{H}$ & 10.73 & -1.48 & $15.4(0.7)$ & 0.05 & 1 \\
\hline $0655-390$ & $6311(211)$ & $8.00(0.00)$ & $0.59(0.00)$ & $\mathrm{H}$ & 13.95 & -3.64 & $17.1(0.7)$ & 1.97 & 2 \\
\hline $0657+320$ & $4888(84)$ & $8.03(0.03)$ & $0.60(0.02)$ & $\mathrm{H}$ & 15.26 & -4.10 & $18.7(0.3)$ & 6.55 & 2 \\
\hline $0659-063$ & $6627(105)$ & $8.36(0.10)$ & $0.82(0.07)$ & $\mathrm{H}$ & 14.36 & -3.77 & $12.3(1.3)$ & 3.69 & 1 \\
\hline $0708-670$ & $5097(116)$ & $8.00(0.00)$ & $0.57(0.00)$ & $\mathrm{He}$ & 15.03 & -4.02 & $17.3(0.6)$ & 5.67 & 2 \\
\hline $0727+482 \mathrm{~A}$ & $4934(81)$ & $7.89(0.02)$ & $0.51(0.01)$ & $\mathrm{H}$ & 15.03 & -4.01 & $11.1(0.1)$ & 4.68 & 2 \\
\hline $0727+482 \mathrm{~B}$ & $4926(85)$ & $8.10(0.01)$ & $0.65(0.01)$ & $\mathrm{H}$ & 15.33 & -4.13 & $11.1(0.1)$ & 7.13 & 2 \\
\hline $0728+642$ & $5135(71)$ & $8.00(0.00)$ & $0.58(0.00)$ & $\mathrm{H}$ & 14.96 & -4.00 & $18.4(0.5)$ & 5.00 & 2 \\
\hline $0736+053$ & 7871 (433) & $8.09(0.00)$ & $0.63(0.00)$ & $\mathrm{He}$ & 13.22 & -3.31 & $3.50(0.01)$ & 1.37 & 2 \\
\hline $0738-172$ & $7650(226)$ & $8.06(0.04)$ & $0.62(0.02)$ & $\mathrm{He}$ & 13.31 & -3.35 & $8.90(0.21)$ & 1.44 & 2 \\
\hline $0743-336$ & $4462(85)$ & $7.96(0.01)$ & $0.55(0.01)$ & $\mathrm{H}$ & 15.69 & -4.23 & $15.2(0.1)$ & 7.32 & 2 \\
\hline $0747+073.1$ & $4366(84)$ & $7.83(0.02)$ & $0.48(0.01)$ & $\mathrm{H}$ & 15.65 & -4.20 & $18.3(0.2)$ & 6.20 & 2 \\
\hline $0747+073.2$ & $4782(85)$ & $7.98(0.02)$ & $0.56(0.01)$ & $\mathrm{H}$ & 15.32 & -4.11 & $18.3(0.2)$ & 6.39 & 2 \\
\hline $0749+426^{*}$ & $4585(74)$ & $8.00(0.00)$ & $0.58(0.00)$ & $\mathrm{H}$ & 16.13 & -4.20 & $24.6(0.8)$ & 7.30 & 2 \\
\hline $0751-252$ & $5085(139)$ & $8.01(0.03)$ & $0.59(0.02)$ & $\mathrm{H}$ & 15.03 & -4.02 & $17.7(0.3)$ & 5.37 & 2 \\
\hline $0752-676$ & $5735(103)$ & $8.23(0.08)$ & $0.73(0.06)$ & $\mathrm{H}$ & 14.70 & -3.94 & $7.08(0.43)$ & 4.50 & 2 \\
\hline $0806-661$ & $10,205(390)$ & $8.00(0.05)$ & $0.58(0.03)$ & $\mathrm{He}$ & 12.30 & -2.80 & $19.2(0.6)$ & 0.62 & 2 \\
\hline $0810+489$ & $6662(141)$ & $8.00(0.00)$ & $0.57(0.00)$ & $\mathrm{He}$ & 13.86 & -3.55 & $18.3(0.6)$ & 1.87 & 2 \\
\hline $0816-310$ & $6463(370)$ & $8.00(0.00)$ & $0.57(0.00)$ & $\mathrm{He}$ & 13.71 & -3.61 & $22.1(1.6)$ & 2.02 & 2 \\
\hline $0821-669$ & $5088(137)$ & $8.12(0.02)$ & $0.66(0.01)$ & $\mathrm{H}$ & 15.20 & -4.08 & $10.7(0.1)$ & 6.58 & 2 \\
\hline $0827+328$ & $7209(178)$ & $8.39(0.11)$ & $0.84(0.07)$ & $\mathrm{H}$ & 13.99 & -3.64 & $22.3(1.9)$ & 3.10 & 2 \\
\hline $0839-327$ & $9081(323)$ & $7.71(0.14)$ & $0.44(0.07)$ & $\mathrm{H}$ & 12.12 & -2.84 & $8.87(0.77)$ & 0.55 & 2 \\
\hline $0840-136$ & $4874(177)$ & $8.00(0.00)$ & $0.57(0.00)$ & $\mathrm{He}$ & 15.01 & -4.10 & $13.9(0.8)$ & 6.31 & 2 \\
\hline $0843+358^{*}$ & 9041 (419) & $8.00(0.00)$ & $0.58(0.00)$ & $\mathrm{He}$ & 12.59 & -3.02 & $27.0(1.5)$ & 0.86 & 2 \\
\hline
\end{tabular}


Table 2-Continued

\begin{tabular}{|c|c|c|c|c|c|c|c|c|c|}
\hline WD & $T_{\text {eff }}(\mathrm{K})$ & $\log g$ & $M / M_{\odot}$ & Comp. & $M_{V}$ & $\log L / L_{\odot}$ & $D(\mathrm{pc})$ & Age (Gyr) & Notes \\
\hline $0856+331$ & $9986(244)$ & $8.73(0.08)$ & $1.05(0.05)$ & $\mathrm{He}$ & 13.60 & -3.32 & $20.5(1.4)$ & 2.09 & 2 \\
\hline $0912+536$ & 7235 (195) & $8.28(0.03)$ & $0.75(0.02)$ & $\mathrm{He}$ & 13.78 & -3.57 & $10.3(0.2)$ & 2.45 & 2 \\
\hline $0946+534^{*}$ & $8161(245)$ & $8.26(0.11)$ & $0.74(0.08)$ & $\mathrm{He}$ & 13.37 & -3.35 & $23.0(1.9)$ & 1.59 & 2 \\
\hline $0955+247^{*}$ & $8634(229)$ & $8.26(0.15)$ & $0.76(0.10)$ & $\mathrm{H}$ & 13.12 & -3.24 & $24.4(2.7)$ & 1.30 & 2 \\
\hline $1008+290$ & $4562(161)$ & $8.17(0.01)$ & $0.68(0.01)$ & $\mathrm{He}$ & 16.66 & -4.31 & $14.8(0.1)$ & 7.58 & 2 \\
\hline $1009-184$ & $6036(364)$ & $8.02(0.03)$ & $0.59(0.02)$ & $\mathrm{He}$ & 14.13 & -3.74 & $18.3(0.3)$ & 2.63 & 2 \\
\hline $1019+637$ & $6742(155)$ & $7.97(0.09)$ & $0.57(0.05)$ & $\mathrm{H}$ & 13.63 & -3.50 & $16.3(1.0)$ & 1.60 & 2 \\
\hline $1033+714$ & $4727(87)$ & $8.00(0.00)$ & $0.58(0.00)$ & $\mathrm{H}$ & 15.41 & -4.15 & $19.6(0.8)$ & 6.82 & 2 \\
\hline $1036-204$ & $4694(124)$ & $8.05(0.02)$ & $0.60(0.01)$ & $\mathrm{He}$ & 15.51 & -4.19 & $14.3(0.1)$ & 7.13 & 2 \\
\hline $1043-188$ & $5780(85)$ & $7.93(0.19)$ & $0.53(0.11)$ & $\mathrm{He}$ & 14.30 & -3.77 & $17.6(2.0)$ & 2.62 & 2 \\
\hline $1055-072$ & 7491 (204) & $8.42(0.06)$ & $0.85(0.04)$ & $\mathrm{He}$ & 13.91 & -3.60 & $12.2(0.5)$ & 2.93 & 2 \\
\hline $1116-470$ & $5801(176)$ & $8.00(0.00)$ & $0.57(0.00)$ & $\mathrm{He}$ & 14.31 & -3.80 & $17.5(0.7)$ & 2.94 & 2 \\
\hline $1121+216$ & 7434 (185) & $8.19(0.05)$ & $0.71(0.03)$ & $\mathrm{H}$ & 13.57 & -3.46 & $13.4(0.5)$ & 1.77 & 2 \\
\hline $1124+595^{*}$ & $10,747(156)$ & $8.60(0.05)$ & $0.98(0.03)$ & $\mathrm{H}$ & 12.93 & -3.09 & $27.6(1.3)$ & 1.47 & 1 \\
\hline $1134+300$ & $22,469(342)$ & $8.56(0.05)$ & $0.97(0.03)$ & $\mathrm{H}$ & 11.48 & -1.78 & $15.3(0.7)$ & 0.16 & 1 \\
\hline $1142-645$ & 7966 (219) & $8.06(0.02)$ & $0.61(0.01)$ & $\mathrm{He}$ & 13.17 & -3.27 & $4.62(0.04)$ & 1.29 & 2 \\
\hline $1202-232$ & 8767 (126) & $7.99(0.05)$ & $0.59(0.03)$ & $\mathrm{H}$ & 12.67 & -3.05 & $10.8(0.4)$ & 0.83 & 1 \\
\hline $1208+576$ & $5870(111)$ & $7.96(0.15)$ & $0.56(0.09)$ & $\mathrm{H}$ & 14.23 & -3.74 & $20.4(1.9)$ & 2.22 & 2 \\
\hline $1223-659$ & $7594(108)$ & $7.73(0.05)$ & $0.45(0.02)$ & $\mathrm{H}$ & 12.89 & -3.16 & $16.3(0.6)$ & 0.87 & 1 \\
\hline $1236-495$ & $11,599(475)$ & $8.62(0.18)$ & $1.00(0.11)$ & $\mathrm{H}$ & 12.73 & -2.97 & $16.4(2.6)$ & 1.21 & 2 \\
\hline $1242-105^{*}$ & $8261(120)$ & $7.75(0.06)$ & $0.46(0.03)$ & $\mathrm{H}$ & 12.57 & -3.03 & $23.5(1.1)$ & 0.72 & 1 \\
\hline $1257+037$ & $5616(100)$ & $8.19(0.09)$ & $0.70(0.06)$ & $\mathrm{H}$ & 14.74 & -3.95 & $16.6(1.0)$ & 4.52 & 2 \\
\hline $1309+853$ & $5440(98)$ & $8.20(0.03)$ & $0.71(0.02)$ & $\mathrm{H}$ & 14.89 & -4.01 & $16.5(0.3)$ & 5.45 & 2 \\
\hline $1310+583^{*}$ & $10,682(156)$ & $8.10(0.05)$ & $0.66(0.03)$ & $\mathrm{H}$ & 12.11 & -2.77 & $24.9(1.0)$ & 0.58 & 1 \\
\hline $1310-472$ & $4158(72)$ & $8.09(0.06)$ & $0.63(0.04)$ & $\mathrm{H}$ & 16.24 & -4.42 & $15.0(0.5)$ & 9.31 & 2 \\
\hline $1315-781$ & $5619(193)$ & $8.17(0.02)$ & $0.69(0.02)$ & $\mathrm{H}$ & 14.75 & -3.94 & $19.2(0.3)$ & 4.39 & 2 \\
\hline $1327-083$ & $14,571(235)$ & $7.99(0.04)$ & $0.61(0.03)$ & $\mathrm{H}$ & 11.31 & -2.16 & $18.0(0.6)$ & 0.21 & 1 \\
\hline $1334+039$ & $4971(83)$ & $7.94(0.05)$ & $0.54(0.03)$ & $\mathrm{H}$ & 15.05 & -4.02 & $8.24(0.23)$ & 5.02 & 2 \\
\hline $1339-340$ & $5258(166)$ & $8.00(0.00)$ & $0.58(0.00)$ & $\mathrm{H}$ & 14.82 & -3.96 & $21.0(1.2)$ & 4.35 & 2 \\
\hline $1344+106$ & 7059 (168) & $8.09(0.10)$ & $0.65(0.07)$ & $\mathrm{H}$ & 13.61 & -3.49 & $20.0(1.5)$ & 1.69 & 2 \\
\hline $1345+238$ & $4581(85)$ & $7.77(0.04)$ & $0.45(0.02)$ & $\mathrm{H}$ & 15.30 & -4.08 & $12.1(0.3)$ & 4.72 & 2 \\
\hline 1350-090* & $9580(136)$ & $8.13(0.05)$ & $0.68(0.03)$ & $\mathrm{H}$ & 12.54 & -2.98 & $25.3(1.0)$ & 0.81 & 1 \\
\hline $1425-811^{*}$ & $12,334(182)$ & $8.00(0.04)$ & $0.61(0.03)$ & $\mathrm{H}$ & 11.60 & -2.46 & $26.9(1.0)$ & 0.35 & 1 \\
\hline $1444-174$ & $4982(78)$ & $8.37(0.08)$ & $0.82(0.05)$ & $\mathrm{H}$ & 15.63 & -4.27 & $14.5(0.8)$ & 8.56 & 2 \\
\hline $1529+141^{*}$ & $8856(355)$ & $8.00(0.00)$ & $0.60(0.00)$ & $\mathrm{H}$ & 12.64 & -3.04 & $60.7(3.6)$ & 0.83 & 2 \\
\hline
\end{tabular}


Table 2-Continued

\begin{tabular}{|c|c|c|c|c|c|c|c|c|c|}
\hline WD & $T_{\text {eff }}(\mathrm{K})$ & $\log g$ & $M / M_{\odot}$ & Comp. & $M_{V}$ & $\log L / L_{\odot}$ & $D(\mathrm{pc})$ & Age (Gyr) & Notes \\
\hline $1538+333^{*}$ & 8899 (127) & $8.05(0.05)$ & $0.63(0.03)$ & $\mathrm{H}$ & 12.71 & -3.06 & $29.1(1.1)$ & 0.88 & 1 \\
\hline $1544-377$ & $10,610(151)$ & $7.91(0.04)$ & $0.55(0.03)$ & $\mathrm{H}$ & 11.84 & -2.67 & $15.2(0.6)$ & 0.46 & 1 \\
\hline $1609+135$ & $9041(259)$ & $8.74(0.10)$ & $1.07(0.06)$ & $\mathrm{H}$ & 13.79 & -3.50 & $18.4(1.6)$ & 2.71 & 2 \\
\hline $1620-391$ & $25,985(369)$ & $7.96(0.04)$ & $0.61(0.02)$ & $\mathrm{H}$ & 10.22 & -1.12 & $12.8(0.5)$ & 0.02 & 1 \\
\hline $1626+368$ & $8507(320)$ & $8.00(0.05)$ & $0.58(0.03)$ & $\mathrm{He}$ & 12.82 & -3.13 & $15.9(0.5)$ & 1.02 & 2 \\
\hline $1632+177$ & $10,225(145)$ & $7.73(0.04)$ & $0.46(0.02)$ & $\mathrm{H}$ & 11.72 & -2.64 & $18.7(0.7)$ & 0.41 & 1 \\
\hline $1633+433$ & $6608(148)$ & $8.14(0.06)$ & $0.68(0.04)$ & $\mathrm{H}$ & 13.94 & -3.63 & $15.1(0.7)$ & 2.31 & 2 \\
\hline $1633+572$ & $5958(116)$ & $8.00(0.06)$ & $0.57(0.04)$ & $\mathrm{He}$ & 14.19 & -3.75 & $14.4(0.5)$ & 2.62 & 2 \\
\hline $1647+591$ & $12,738(201)$ & $8.24(0.05)$ & $0.76(0.03)$ & $\mathrm{H}$ & 11.90 & -2.55 & $11.0(0.5)$ & 0.45 & 1 \\
\hline $1653+385^{*}$ & $5833(126)$ & $8.00(0.00)$ & $0.59(0.00)$ & $\mathrm{H}$ & 14.56 & -3.77 & $30.7(1.2)$ & 2.43 & 2 \\
\hline $1655+215^{*}$ & $9179(241)$ & $7.87(0.11)$ & $0.52(0.06)$ & $\mathrm{H}$ & 12.30 & -2.90 & $23.3(1.7)$ & 0.63 & 2 \\
\hline $1657+321^{*}$ & $6370(183)$ & $8.00(0.00)$ & $0.59(0.00)$ & $\mathrm{H}$ & 14.09 & -3.62 & $51.7(2.5)$ & 1.92 & 2 \\
\hline $1705+030$ & $6584(207)$ & $8.18(0.14)$ & $0.68(0.09)$ & $\mathrm{He}$ & 13.98 & -3.67 & $17.5(1.7)$ & 2.75 & 2 \\
\hline $1729+371^{*}$ & $10,560(149)$ & $8.10(0.04)$ & $0.64(0.03)$ & $\mathrm{H}+\mathrm{He}$ & 12.32 & -2.80 & $50.3(2.2)$ & 0.65 & 1 \\
\hline $1748+708$ & $5570(107)$ & $8.34(0.02)$ & $0.79(0.01)$ & $\mathrm{He}$ & 15.21 & -4.07 & $6.07(0.09)$ & 5.86 & 2 \\
\hline $1756+143$ & $5167(175)$ & $8.00(0.00)$ & $0.58(0.00)$ & $\mathrm{H}$ & 14.74 & -3.99 & $20.5(1.2)$ & 4.84 & 2 \\
\hline $1756+827$ & $7214(146)$ & $7.97(0.07)$ & $0.58(0.04)$ & $\mathrm{H}$ & 13.37 & -3.39 & $15.7(0.7)$ & 1.36 & 2 \\
\hline $1814+134$ & $5251(155)$ & $8.15(0.03)$ & $0.68(0.02)$ & $\mathrm{H}$ & 15.09 & -4.05 & $14.2(0.2)$ & 6.05 & 2 \\
\hline $1820+609$ & $4919(73)$ & $7.97(0.09)$ & $0.56(0.05)$ & $\mathrm{H}$ & 15.16 & -4.06 & $12.8(0.7)$ & 5.68 & 2 \\
\hline $1829+547$ & $6345(136)$ & $8.50(0.11)$ & $0.90(0.07)$ & $\mathrm{He}$ & 14.69 & -3.94 & $15.0(1.3)$ & 4.65 & 2 \\
\hline $1900+705$ & $11,835(460)$ & $8.53(0.04)$ & $0.93(0.02)$ & $\mathrm{He}$ & 12.68 & -2.88 & $13.0(0.4)$ & 0.91 & 2 \\
\hline $1917+386$ & $6459(143)$ & $8.28(0.05)$ & $0.75(0.04)$ & $\mathrm{He}$ & 14.27 & -3.77 & $11.7(0.5)$ & 3.55 & 2 \\
\hline $1917-077$ & $10,396(357)$ & $8.06(0.04)$ & $0.62(0.02)$ & $\mathrm{He}$ & 12.28 & -2.81 & $10.1(0.3)$ & 0.65 & 2 \\
\hline $1919+145$ & $15,280(246)$ & $8.21(0.04)$ & $0.74(0.03)$ & $\mathrm{H}$ & 11.55 & -2.21 & $19.8(0.8)$ & 0.26 & 1 \\
\hline $1935+276$ & $12,631(195)$ & $7.98(0.05)$ & $0.60(0.03)$ & $\mathrm{H}$ & 11.54 & -2.41 & $18.0(0.8)$ & 0.32 & 1 \\
\hline $1953-011$ & $7868(202)$ & $8.23(0.04)$ & $0.73(0.03)$ & $\mathrm{H}$ & 13.41 & -3.38 & $11.4(0.4)$ & 1.63 & 2 \\
\hline $2002-110$ & $4675(88)$ & $8.23(0.02)$ & $0.72(0.01)$ & $\mathrm{H}$ & 15.76 & -4.29 & $17.3(0.2)$ & 8.80 & 2 \\
\hline $2007-303$ & $16,147(233)$ & $7.98(0.04)$ & $0.60(0.02)$ & $\mathrm{H}$ & 11.11 & -1.97 & $15.4(0.6)$ & 0.15 & 1 \\
\hline $2008-600$ & $4905(213)$ & $7.77(0.02)$ & $0.44(0.01)$ & $\mathrm{H}+\mathrm{He}$ & 14.75 & -3.97 & $16.6(0.2)$ & 3.76 & 2 \\
\hline $2032+248$ & $20,704(322)$ & $8.03(0.05)$ & $0.64(0.03)$ & $\mathrm{H}$ & 10.75 & -1.56 & $14.8(0.6)$ & 0.06 & 1 \\
\hline $2039-202$ & $20,163(300)$ & $7.98(0.04)$ & $0.61(0.03)$ & $\mathrm{H}$ & 10.72 & -1.58 & $21.1(0.8)$ & 0.06 & 1 \\
\hline $2039-682$ & $17,105(289)$ & $8.59(0.05)$ & $0.98(0.03)$ & $\mathrm{H}$ & 11.98 & -2.27 & $19.6(0.9)$ & 0.36 & 1 \\
\hline $2040-392^{*}$ & $11,302(163)$ & $8.01(0.04)$ & $0.61(0.03)$ & $\mathrm{H}$ & 11.81 & -2.62 & $22.6(0.9)$ & 0.44 & 1 \\
\hline $2047+372$ & $14,712(286)$ & $8.31(0.04)$ & $0.81(0.03)$ & $\mathrm{H}$ & 11.78 & -2.34 & $17.3(0.7)$ & 0.34 & 1 \\
\hline $2048+263$ & $5073(76)$ & $7.25(0.13)$ & $0.24(0.04)$ & $\mathrm{H}$ & 14.12 & -3.65 & $20.1(1.4)$ & 1.60 & 2 \\
\hline
\end{tabular}


Table 2-Continued

\begin{tabular}{|c|c|c|c|c|c|c|c|c|c|}
\hline WD & $T_{\text {eff }}(\mathrm{K})$ & $\log g$ & $M / M_{\odot}$ & Comp. & $M_{V}$ & $\log L / L_{\odot}$ & $D(\mathrm{pc})$ & Age (Gyr) & Notes \\
\hline $2048-250^{*}$ & $7612(221)$ & $8.00(0.00)$ & $0.59(0.00)$ & $\mathrm{H}$ & 13.17 & -3.31 & $28.2(1.1)$ & 1.23 & 2 \\
\hline $2054-050$ & $4340(83)$ & $7.62(0.13)$ & $0.37(0.06)$ & $\mathrm{H}$ & 15.45 & -4.11 & $17.7(1.3)$ & 4.27 & 2 \\
\hline $2105-820$ & $10,218(306)$ & $8.23(0.21)$ & $0.74(0.13)$ & $\mathrm{H}$ & 12.45 & -2.93 & $17.1(2.6)$ & 0.80 & 2 \\
\hline $2115-560 *$ & $9877(141)$ & $7.96(0.05)$ & $0.58(0.03)$ & $\mathrm{H}$ & 12.17 & -2.83 & $26.5(1.0)$ & 0.59 & 1 \\
\hline $2117+539$ & $14,684(239)$ & $7.91(0.05)$ & $0.56(0.03)$ & $\mathrm{H}$ & 11.18 & -2.10 & $19.7(0.7)$ & 0.18 & 1 \\
\hline $2126+734$ & $16,104(237)$ & $7.97(0.04)$ & $0.60(0.03)$ & $\mathrm{H}$ & 11.10 & -1.97 & $21.2(0.8)$ & 0.15 & 1 \\
\hline $2138-332$ & 7399 (388) & $8.19(0.04)$ & $0.70(0.02)$ & $\mathrm{He}$ & 13.50 & -3.48 & $15.6(0.3)$ & 1.87 & 2 \\
\hline $2140+207$ & $8267(255)$ & $7.83(0.07)$ & $0.48(0.04)$ & $\mathrm{He}$ & 12.75 & -3.09 & $12.5(0.5)$ & 0.82 & 2 \\
\hline $2151-015^{*}$ & 9194 (133) & $7.97(0.06)$ & $0.58(0.03)$ & $\mathrm{H}$ & 12.47 & -2.96 & $24.5(1.0)$ & 0.72 & 1 \\
\hline $2154-512$ & $7193(92)$ & $8.03(0.07)$ & $0.60(0.04)$ & $\mathrm{He}$ & 13.69 & -3.44 & $16.2(0.7)$ & 1.63 & 2 \\
\hline $2159-754$ & $8865(130)$ & $8.50(0.06)$ & $0.92(0.04)$ & $\mathrm{H}$ & 13.44 & -3.35 & $21.0(1.1)$ & 2.10 & 1 \\
\hline $2211-392$ & $6150(135)$ & $8.34(0.07)$ & $0.80(0.04)$ & $\mathrm{H}$ & 14.56 & -3.88 & $18.7(0.9)$ & 4.26 & 2 \\
\hline $2215+368^{*}$ & $4993(192)$ & $8.00(0.00)$ & $0.58(0.00)$ & $\mathrm{H}$ & 15.13 & -4.05 & $23.5(1.8)$ & 5.72 & 2 \\
\hline $2226-754$ & $4284(138)$ & $8.00(0.00)$ & $0.58(0.00)$ & $\mathrm{H}$ & 15.93 & -4.32 & $13.5(0.9)$ & 8.25 & 2 \\
\hline $2226-755$ & 4211 (143) & $8.00(0.00)$ & $0.58(0.00)$ & $\mathrm{H}$ & 16.08 & -4.35 & $14.4(1.0)$ & 8.46 & 2 \\
\hline $2246+223$ & $10,375(326)$ & $8.57(0.09)$ & $0.96(0.06)$ & $\mathrm{H}$ & 12.99 & -3.13 & $19.1(1.5)$ & 1.52 & 2 \\
\hline $2248+293$ & $5592(98)$ & $7.55(0.16)$ & $0.35(0.07)$ & $\mathrm{H}$ & 13.94 & -3.62 & $20.9(1.9)$ & 1.51 & 2 \\
\hline $2251-070$ & $4000(0)$ & $8.01(0.06)$ & $0.58(0.03)$ & $\mathrm{He}$ & 16.17 & -4.45 & $8.08(0.29)$ & 8.48 & 2 \\
\hline $2322+137^{*}$ & $5179(71)$ & $7.54(0.08)$ & $0.35(0.03)$ & $\mathrm{H}$ & 14.73 & -3.75 & $22.3(1.0)$ & 1.94 & 2 \\
\hline $2326+049$ & $12,206(187)$ & $8.04(0.05)$ & $0.63(0.03)$ & $\mathrm{H}$ & 11.67 & -2.50 & $13.6(0.8)$ & 0.38 & 1 \\
\hline $2336-079$ & $10,938(317)$ & $8.25(0.03)$ & $0.76(0.02)$ & $\mathrm{H}$ & 12.27 & -2.82 & $15.9(0.4)$ & 0.70 & 2 \\
\hline $2341+322$ & $13,128(198)$ & $7.92(0.04)$ & $0.56(0.03)$ & $\mathrm{H}$ & 11.39 & -2.30 & $17.6(0.8)$ & 0.26 & 1 \\
\hline $2347+292$ & $5805(110)$ & $7.83(0.15)$ & $0.49(0.08)$ & $\mathrm{H}$ & 14.10 & -3.69 & $21.5(1.9)$ & 1.93 & 2 \\
\hline $2351-335^{*}$ & $8810(128)$ & $7.97(0.06)$ & $0.58(0.03)$ & $\mathrm{H}$ & 12.63 & -3.03 & $23.4(1.0)$ & 0.80 & 1 \\
\hline $2359-434$ & 8648 (123) & $8.29(0.05)$ & $0.78(0.03)$ & $\mathrm{H}$ & 13.20 & -3.26 & $7.85(0.40)$ & 1.37 & 1 \\
\hline
\end{tabular}

Note. - (1) Spectroscopic technique; (2) photometric technique. 
Fig. 1.- Blue spectroscopic observations of DA and DAZ stars, shown in order of decreasing effective temperature, from top left to bottom right. All spectra are normalized at $4500 \AA$ and offset from each other by a factor of 0.5 . The last object, 0939+071, is not a white dwarf.

Fig. 2.- Blue spectra of DA and DAZ stars too cool to be analyzed using line profile fitting techniques, shown in order of right ascension. All spectra are normalized to a continuum set to unity and offset from each other by a factor of 0.5 .

Fig. 3.- White dwarfs in our sample whose spectra show $\mathrm{H} \alpha$, shown in order of decreasing equivalent widths, from top left to bottom right. All spectra are normalized to a continuum set to unity, and offset vertically from each other by a factor of 0.4 . The five objects in the right panel are magnetic and exhibit the characteristic Zeeman splitting.

Fig. 4.- Spectroscopic observations of featureless DC stars, shown in order of right ascension. All spectra are normalized to a continuum set to unity and offset from each other by a factor of 0.7 .

Fig. 5.- Spectroscopic observations of normal DQ stars shown in order of approximate molecular band/line strength. All spectra are normalized to a continuum set to unity and offset from each other by a factor of 0.5 . Some of these DQ stars show carbon features only in the ultraviolet.

Fig. 6.- Spectroscopic observations of peculiar DQ stars shown in order of approximate line strength; a normal DQ star, reproduced from Figure 5, is also shown at the top for comparison. All spectra are normalized at $6600 \AA$ and offset from each other by a factor of 0.3 .

Fig. 7.- Blue spectroscopic observations of DZ stars, shown in order of approximate slope. The two objects at the top are actually DZA stars. All spectra are normalized at $4600 \AA$ (4900 $\AA$ for 2251-070) and offset from each other by a factor of 0.7 .

Fig. 8.- Spectroscopic observations of miscellaneous white stars in our sample, discussed in the text. All spectra are normalized at $6400 \AA$ and offset from each other by a factor of 0.2 .

Fig. 9.- $(V-I, V-K)$ two-color diagram for the data set from Table 1. DA and non-DA stars are represented by filled and open circles, respectively, and the cross indicates the size of the average error bar. The pure hydrogen (red line) and pure helium (blue line) model sequences at $\log g=8.0$ are superimposed on the observed data. Temperatures are indicated by small filled circles every $10^{3} \mathrm{~K}$ on the cooling sequence, starting at 12,000 $\mathrm{K}$ at the bottom 
left.

Fig. 10.- $M_{V}$ vs. $(V-I)$ color-magnitude diagram for the data set from Table 1, Objects are split into DA stars (filled circles) and non-DA stars (open circles), based on the presence or absence of $\mathrm{H} \alpha$. The pure hydrogen (red line) and pure helium (blue line) model sequences at $\log g=8.0$ are superimposed on the observed data. Temperatures are indicated by small filled circles every $10^{3} \mathrm{~K}$ on the cooling sequence, starting at 12,000 $\mathrm{K}$ at the upper left of the diagram. The object at the top right of the diagram is LHS 1660 (0419-487), whose colors are contaminated by the presence of an $\mathrm{M}$ dwarf companion.

Fig. 11. - Fits to the observed energy distributions with pure hydrogen models (filled circles) and with pure helium models or mixed hydrogen/helium (open circles), with abundances indicated in each panel. Adopted atmospheric parameters and abundances are emphasized in red. Here and in the following figures, the photometric observations are represented by error bars. In the right panels are shown the observed normalized spectra together with the synthetic line profiles calculated with the atmospheric parameters corresponding to the pure hydrogen solutions.

Fig. 12.- Fits to the observed energy distributions of DQ stars. The filled circles correspond to our best fit with the atmospheric parameters and carbon abundances given in each panel. In the right panels are shown the observed spectra together with the predicted model fit (in red).

Fig. 13.- Best fit to the energy distribution (top) and optical SDSS spectrum (bottom) of GD $184(1529+141)$ assuming an unresolved double degenerate system composed of a DA and a DQ white dwarf at $\log g=8$ for both components. The atmospheric parameters of each component are given in the lower panel; we assume for simplicity that both stars have $\log g=8$. In the top panel, the solid line represents the combined monochromatic model flux, while the contribution of each component is shown as dotted lines (DQ top, DA bottom).

Fig. 14.- Fits to the energy distributions of DZ stars. The filled circles correspond to our best fit with the atmospheric parameters as well as hydrogen and calcium abundances given in each panel. In the right panels are shown the observed spectra together with the predicted model fit (in red); the insert shows our fit to $\mathrm{H} \alpha$, when detected.

Fig. 15. - Fits to the optical spectra of the DA stars in our sample. The lines range from $\mathrm{H} \beta$ (bottom) to $\mathrm{H} 8$ (top), each offset vertically by a factor of 0.2 . Theoretical line profiles shown in green are not used in the fitting procedure. 
Fig. 16. - Top panel: atmospheric parameters for the DA stars in the SDSS, taken from Tremblay et al. (2011a). The red curve corresponds to a third order polynomial fit through the cool $\left(T_{\text {eff }}<14,000 \mathrm{~K}\right)$ objects using temperature bins of $500 \mathrm{~K}$ (see text for details). The dashed line shows a $0.594 M_{\odot}$ evolutionary track, which corresponds to the median mass of the hotter DA stars in this sample. Bottom panel: same results but with the $\log g$ correction applied to the coolest DA stars.

Fig. 17. - Comparison of effective temperatures and absolute magnitudes for the 22 white dwarfs in our sample (all of the DA type) that have both spectroscopic and photometric determinations. The objects labeled in the figure are discussed in the text.

Fig. 18. - Comparison of our distance estimates with those from Sion et al. (2009). The dotted line indicates the 1:1 correspondence, while the dashed line represents the $20 \mathrm{pc}$ limit of the nearby sample defined in this study. Error bars are shown only for stars beyond this distance.

Fig. 19. - Masses of all stars in the local sample $(D \lesssim 20 \mathrm{pc})$ as a function of effective temperature. The various symbols are defined in the legend; known unresolved double degenerate binaries (DD) are shown in red. Also shown are theoretical isochrones labeled in Gyr; solid lines correspond to white dwarf cooling ages only, while the dotted lines also include the main sequence lifetime.

Fig. 20.- Left panel: total number of white dwarfs (solid-line histogram) and hydrogenatmosphere white dwarfs (hatched histogram) as a function of effective temperature. Right panel: ratio of helium-atmosphere white dwarfs to the total number of stars as a function of effective temperature.

Fig. 21. - Mass distribution for the white dwarf stars in the local sample. The individual contributions of the hydrogen-atmosphere (red) and helium-atmosphere (blue) white dwarfs are shown in the left and right panels, respectively. Mean values and standard deviations of the three distributions are indicated in the figure.

Fig. 22.- Luminosity function for our sample of nearby white dwarfs as a function of $M_{\text {bol }}$ (solid line), compared to the luminosity function obtained by Harris et al. (2006) for white dwarfs in the SDSS (dashed line). For the local sample, the number of stars in each magnitude bin is also given. The temperature scale assuming $M=0.6 M_{\odot}$ is also shown at the top of the figure. 


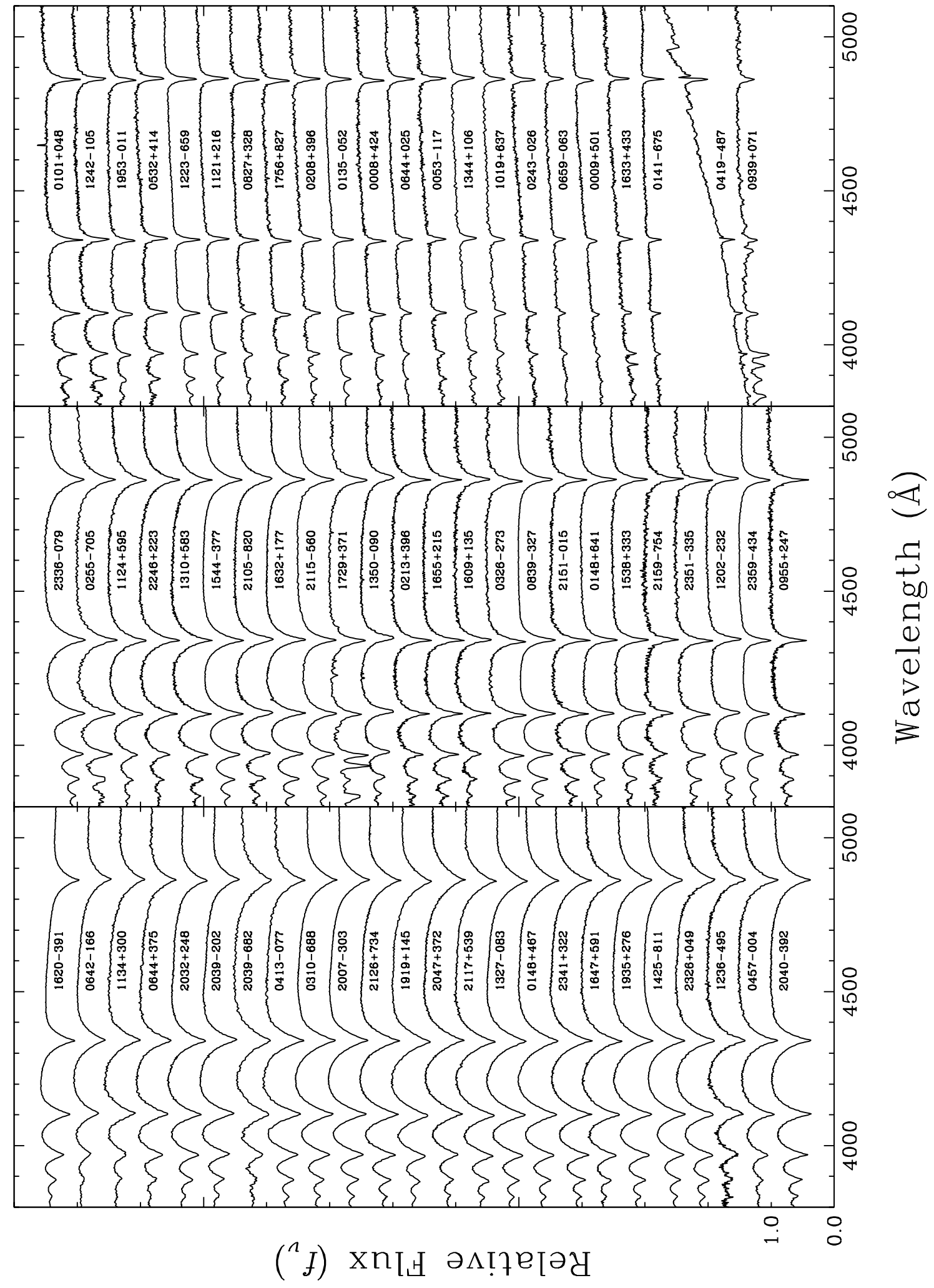

Figure 1] 


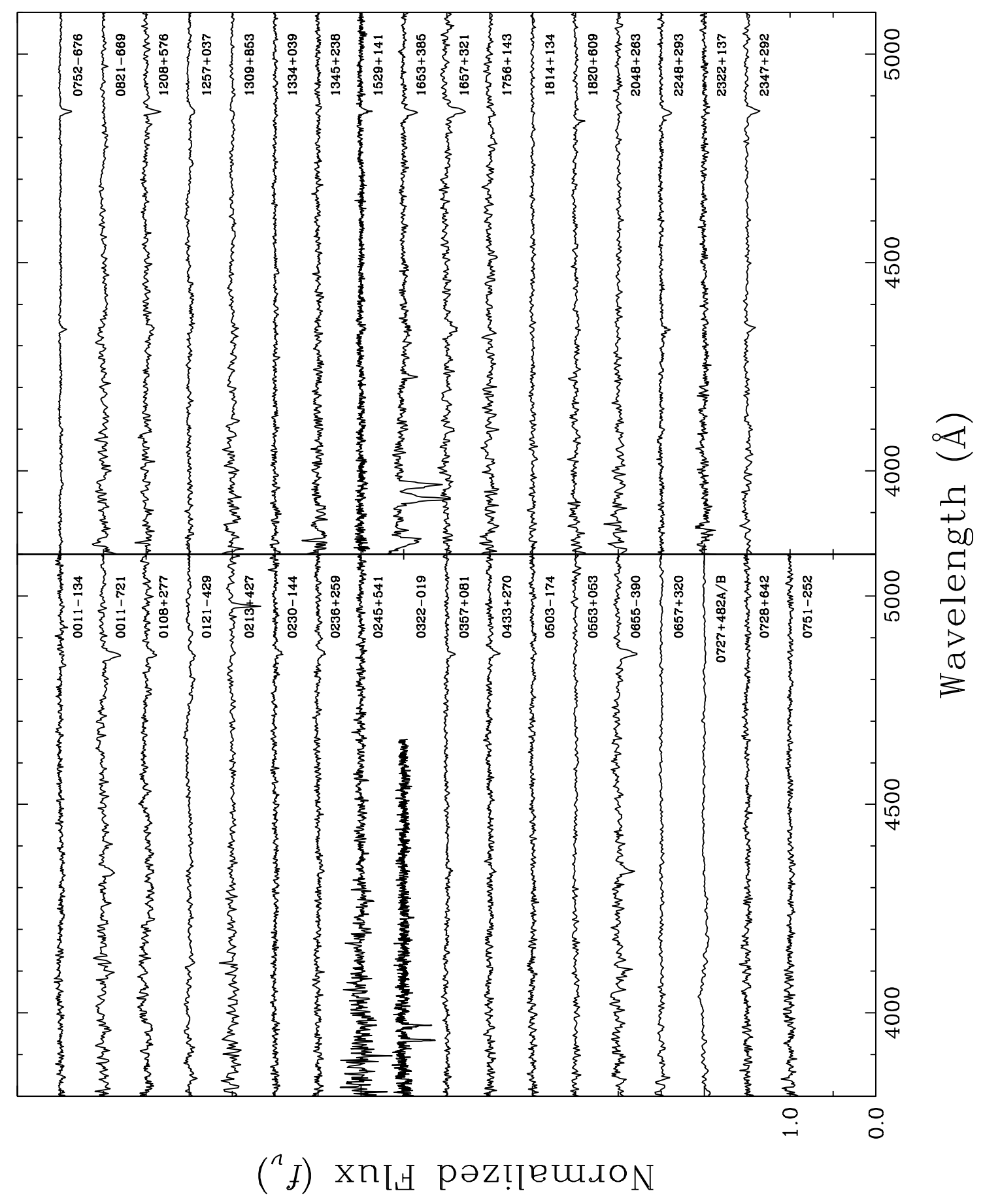

Figure 2 


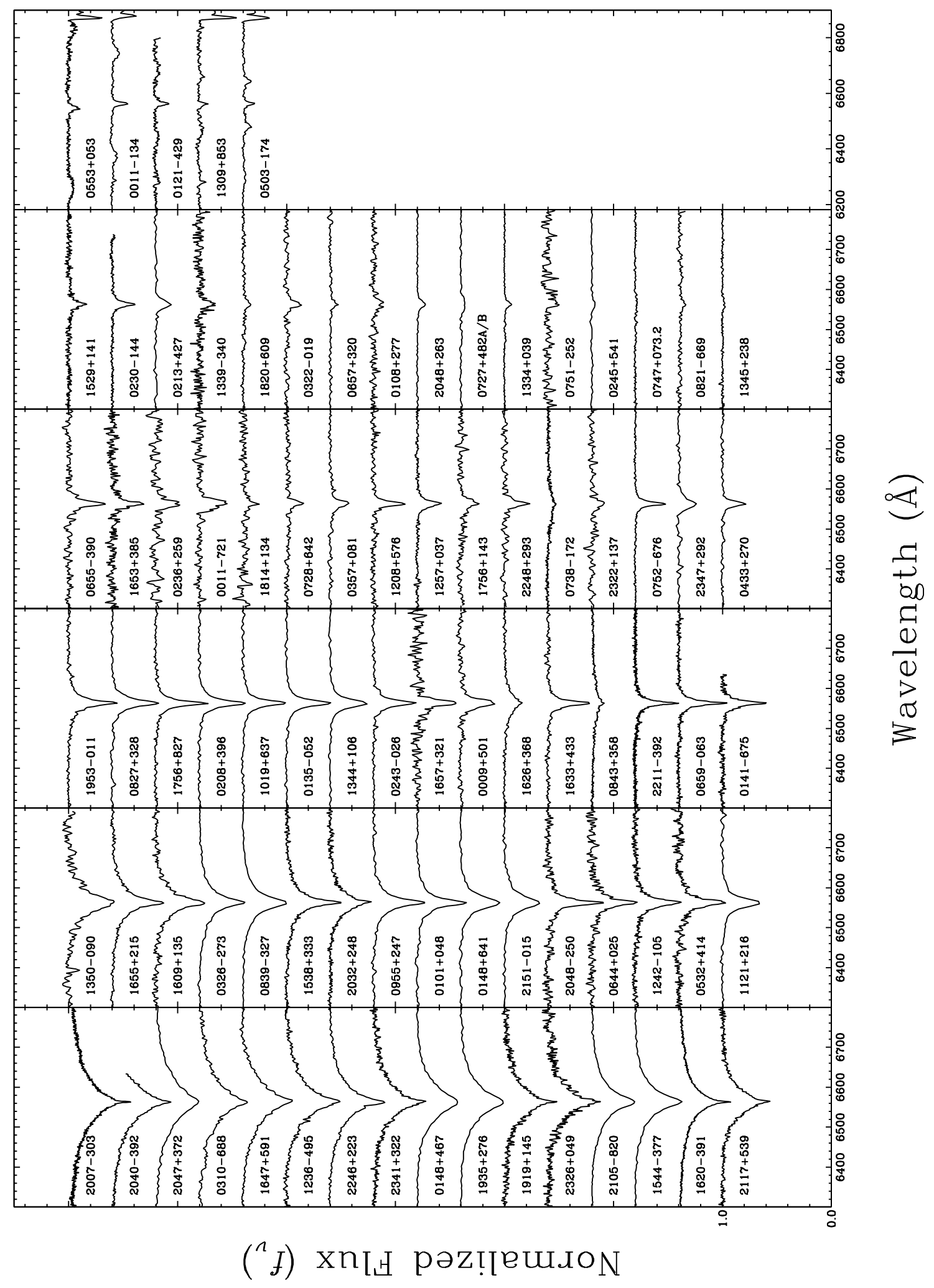

Figure 3 


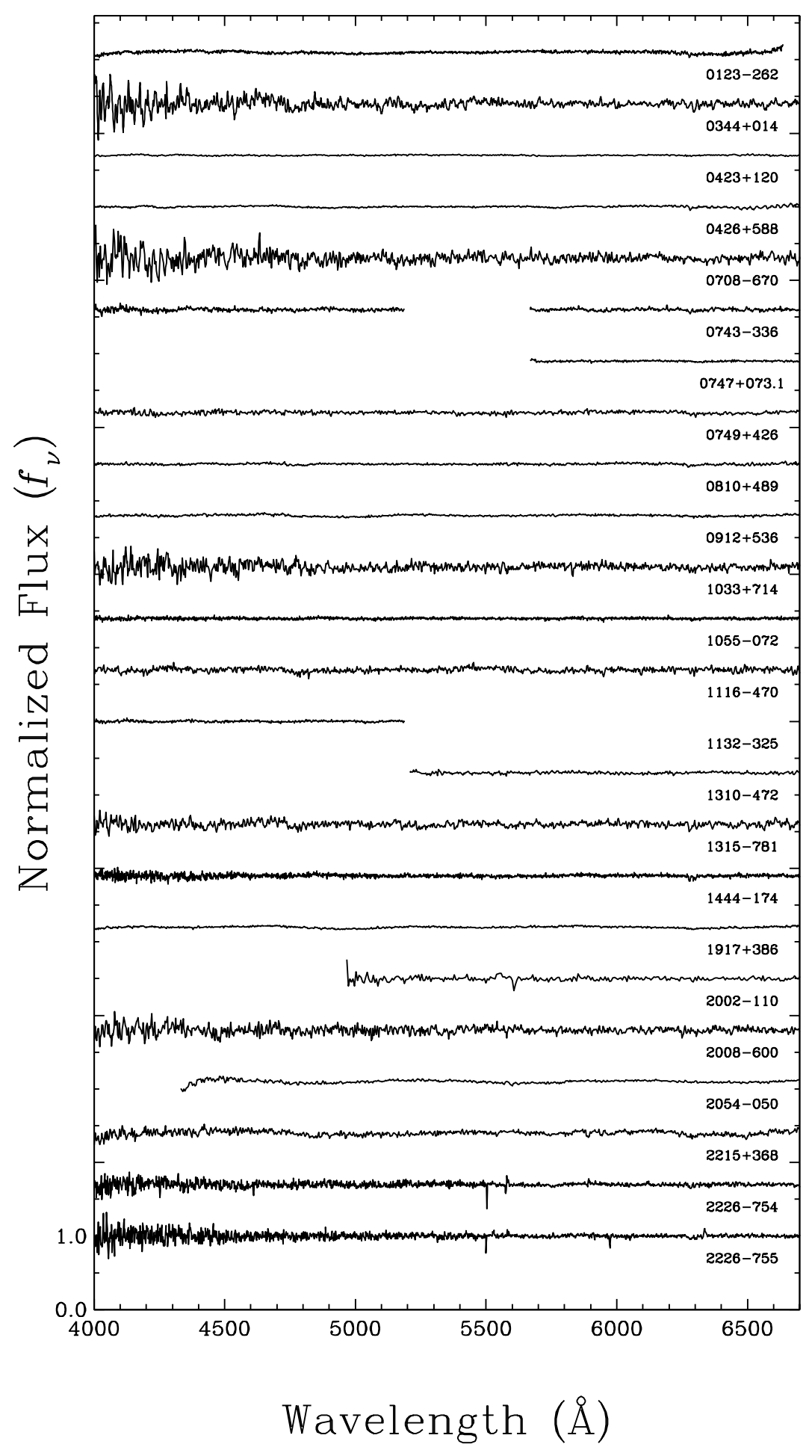

Figure 4 


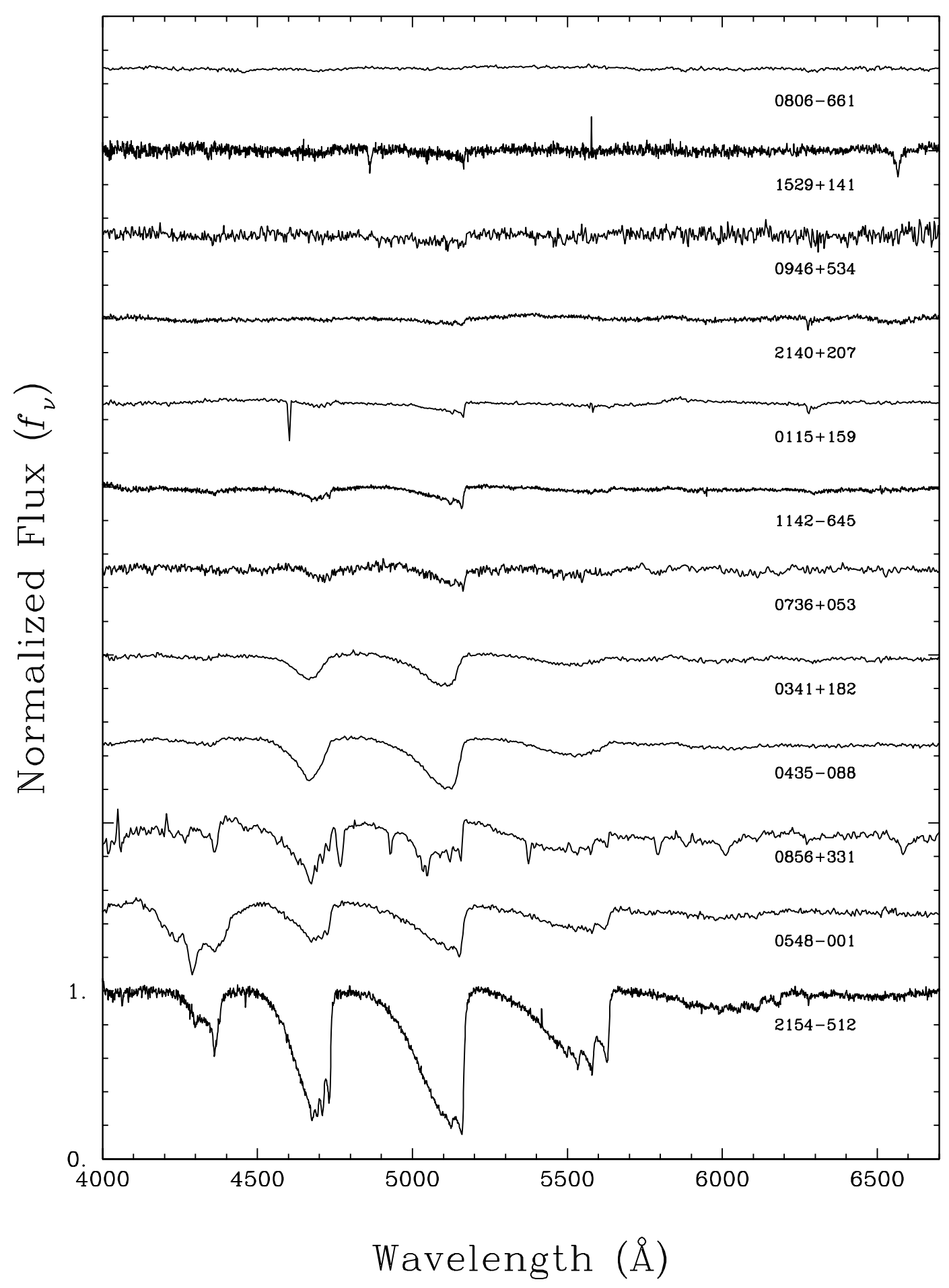

Figure 5 


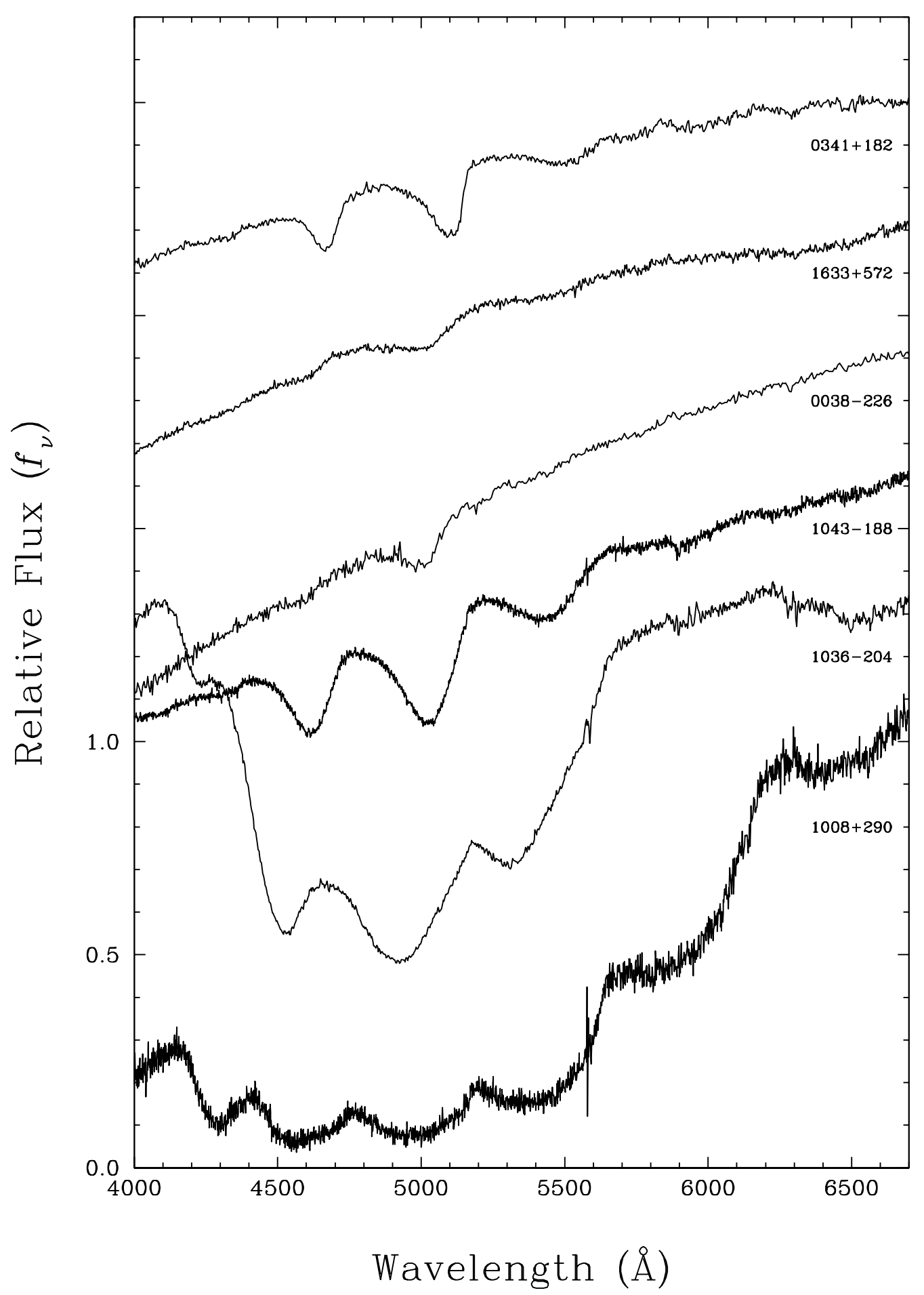

Figure 6 


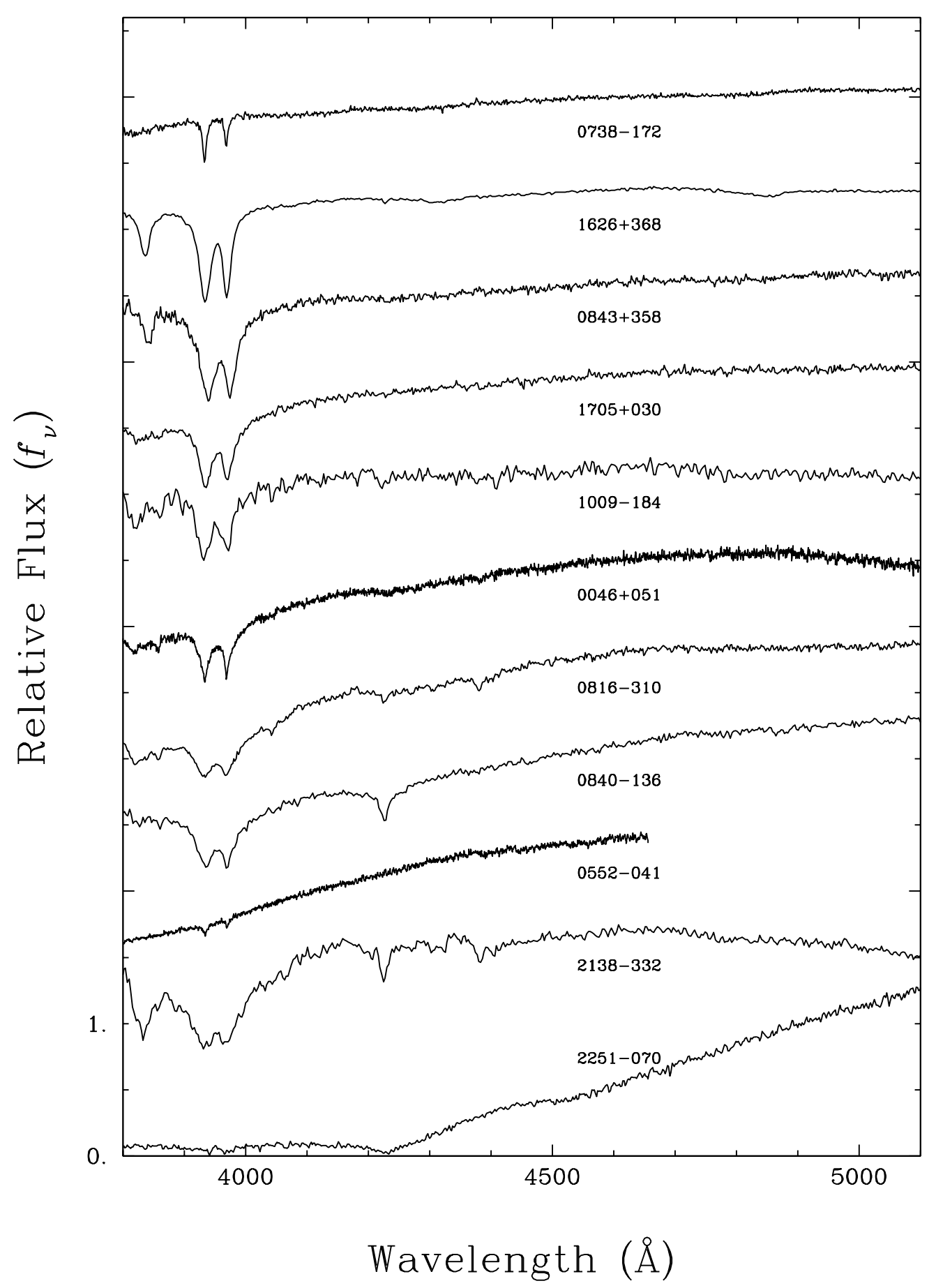

Figure 7 


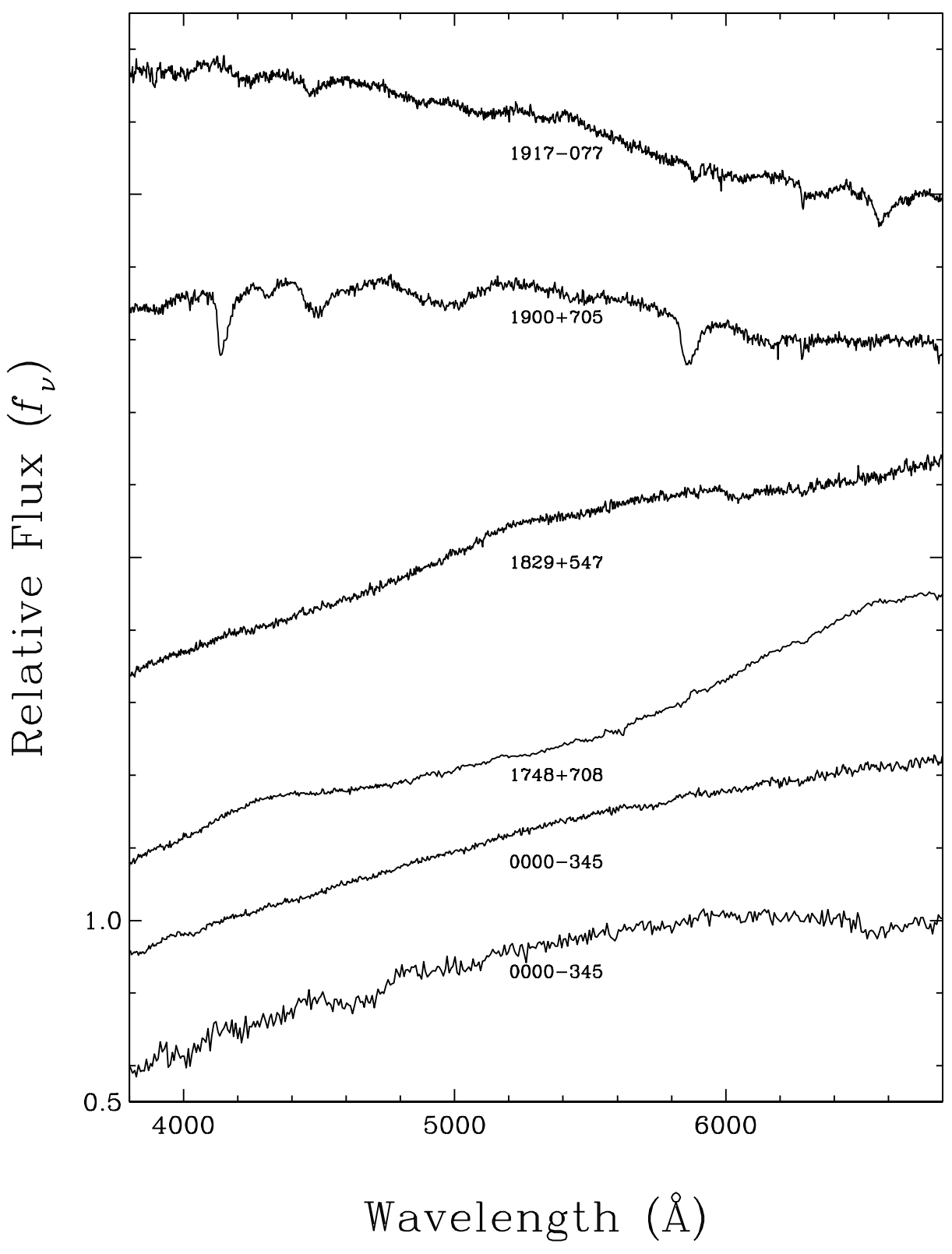

Figure 8 


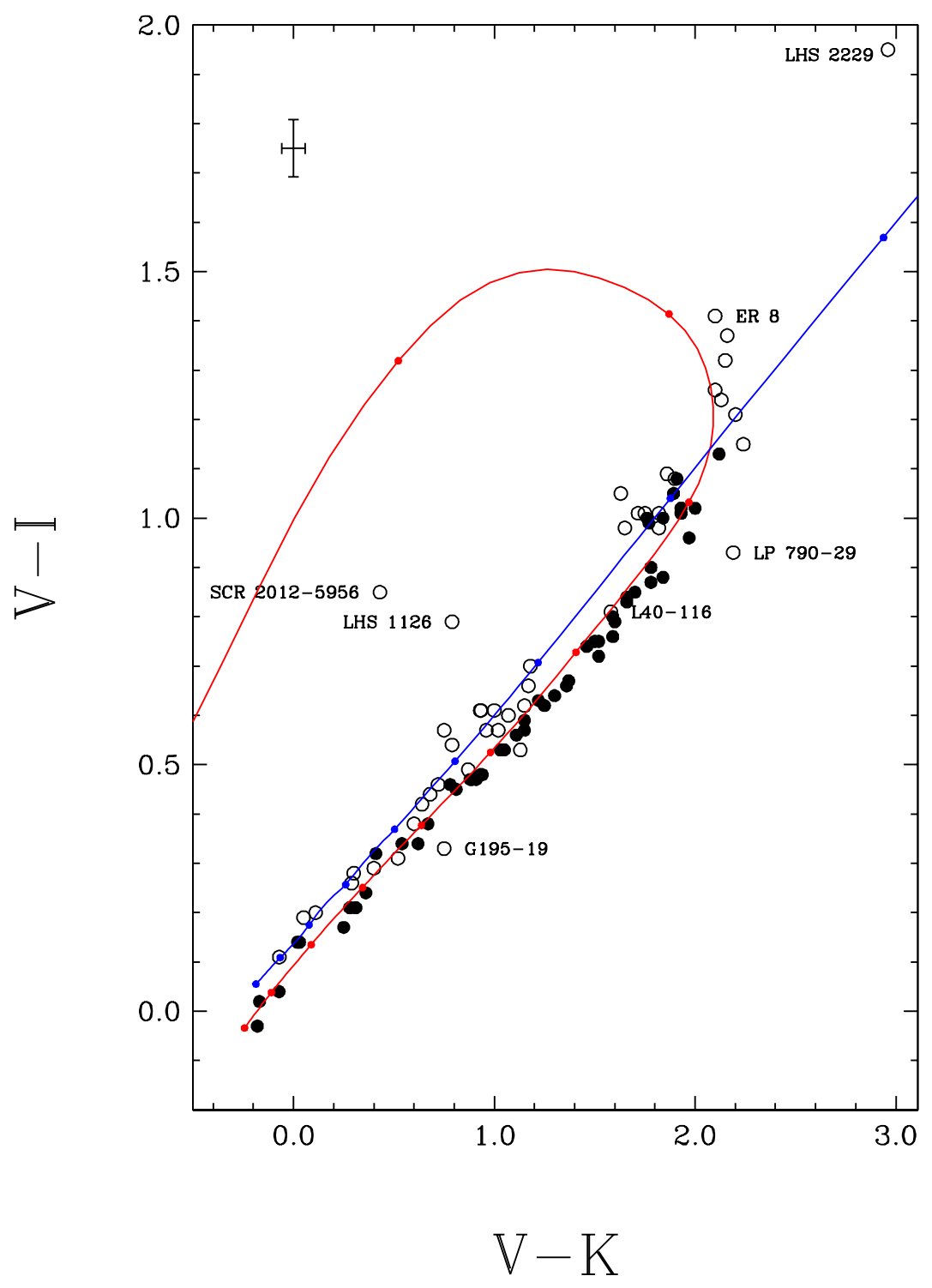

Figure 9 


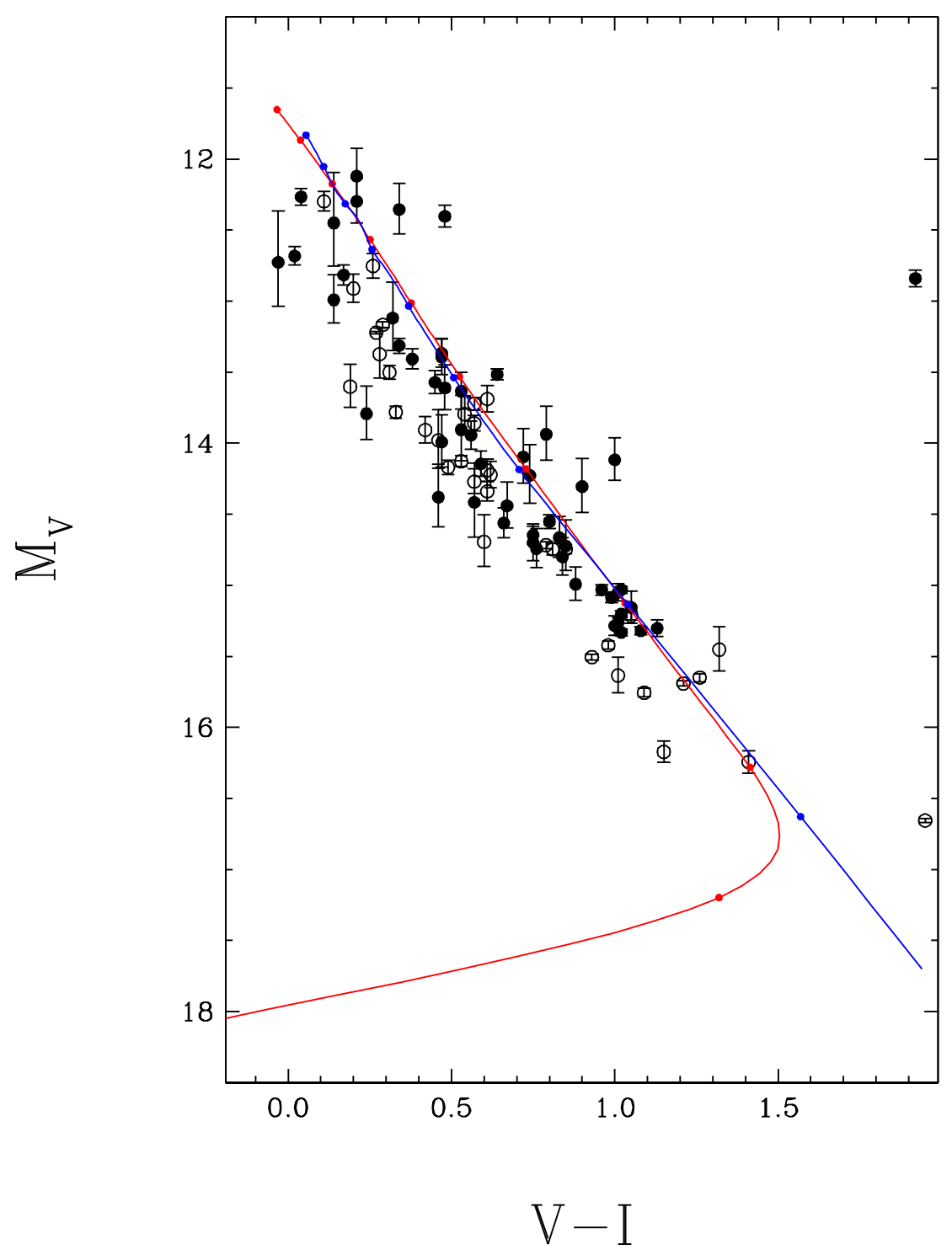

Figure 10 


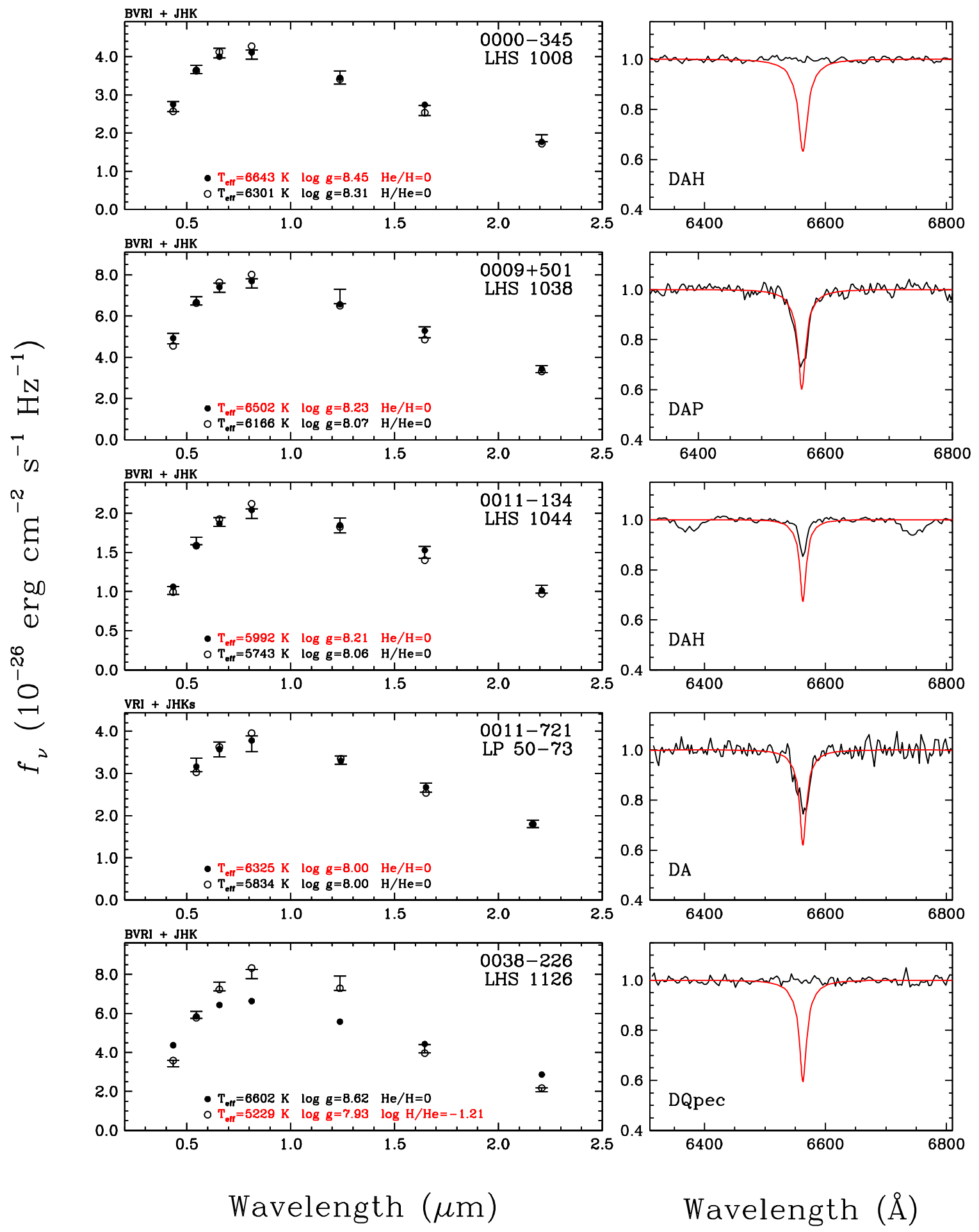

Figure 11a 


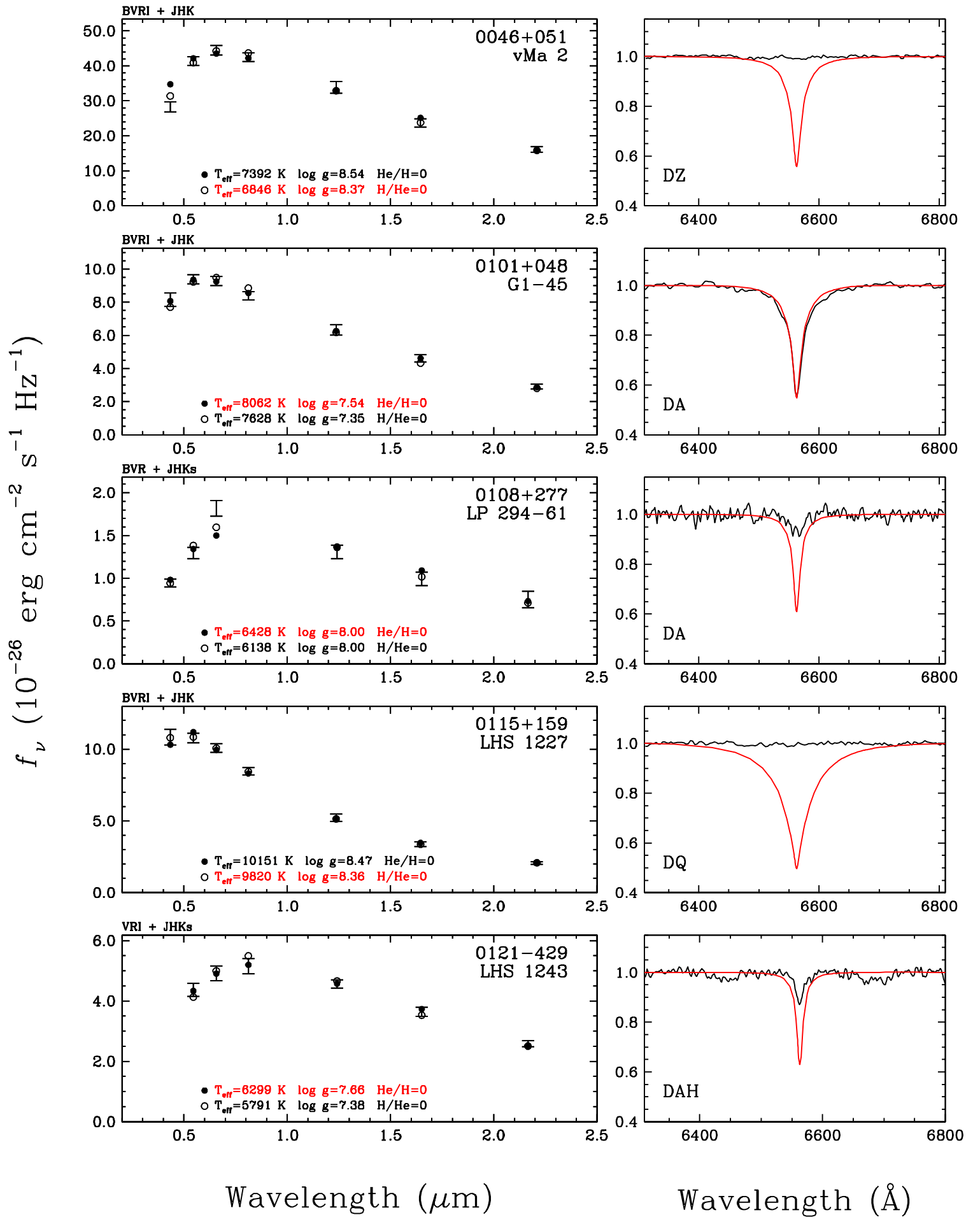

Figure 11b 


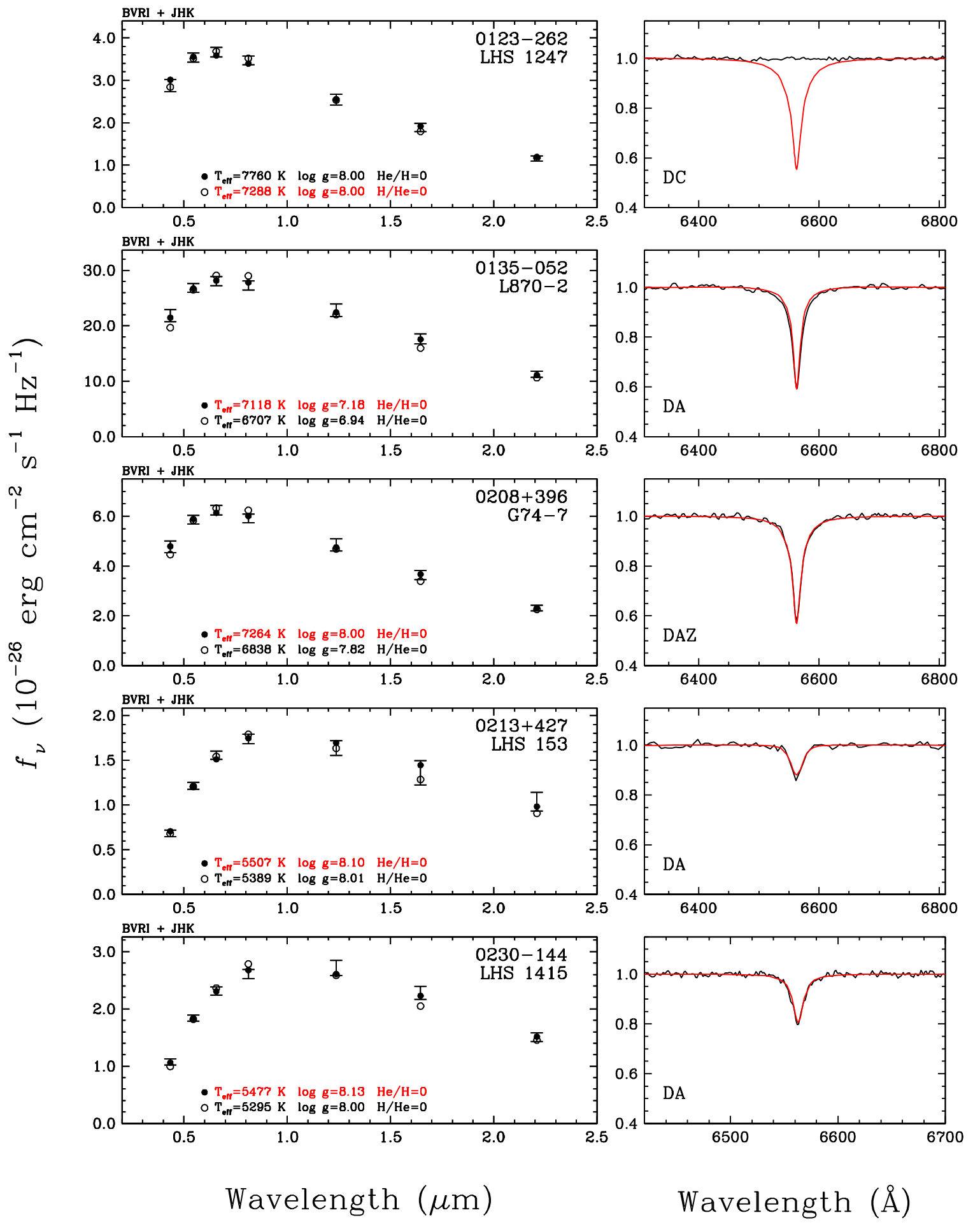

Figure 11k 


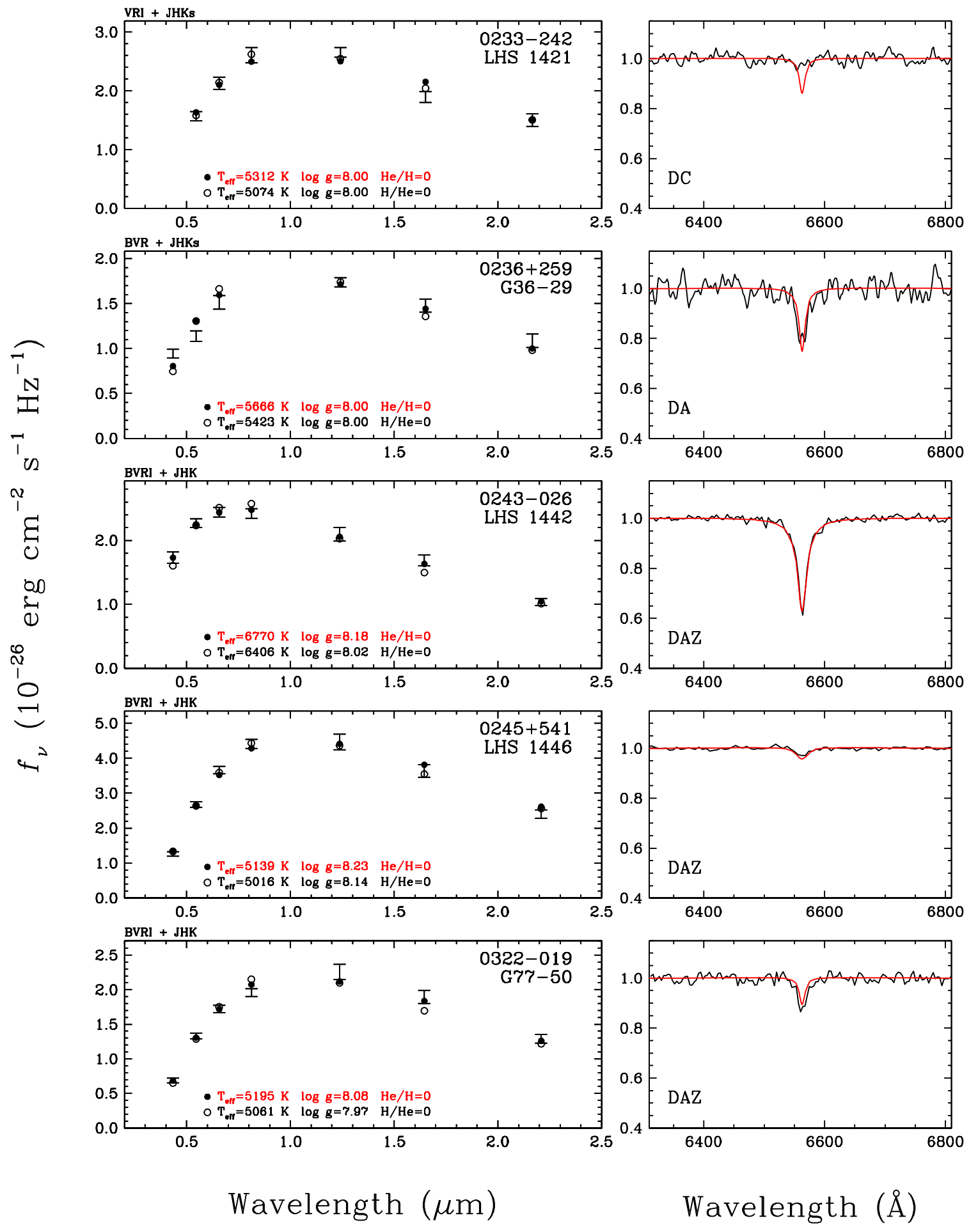

Figure 11d 


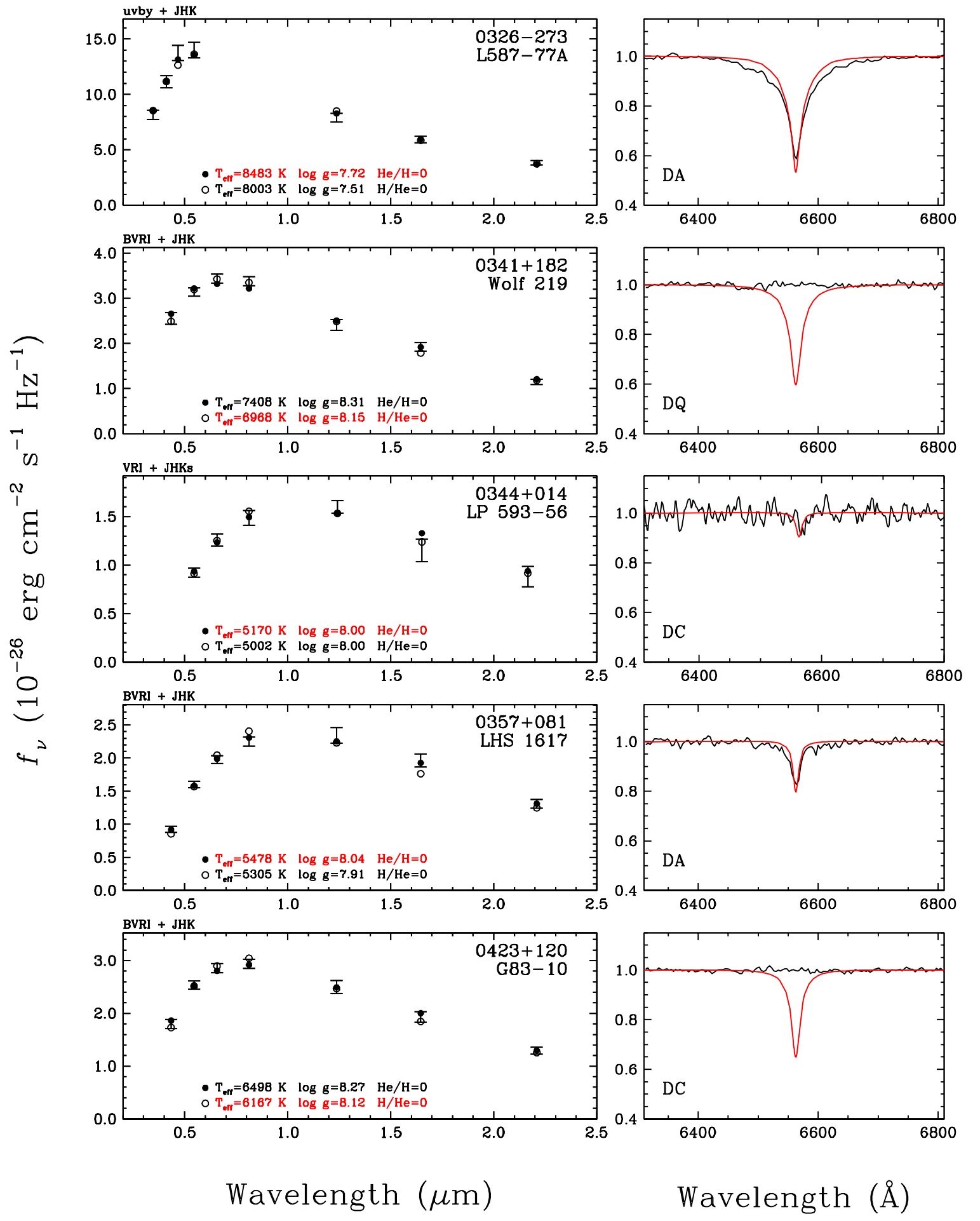

Figure 11e 


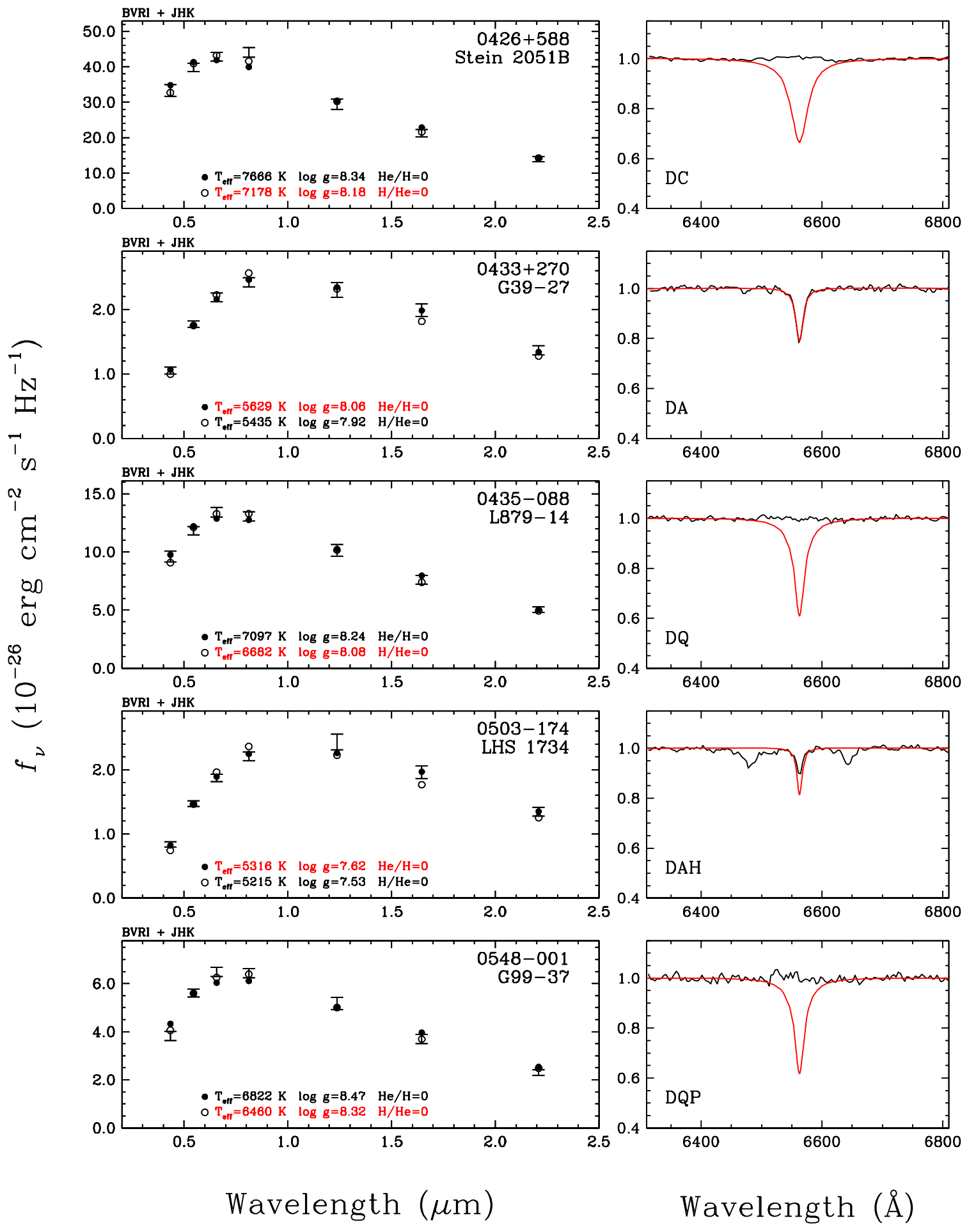

Figure 11f 


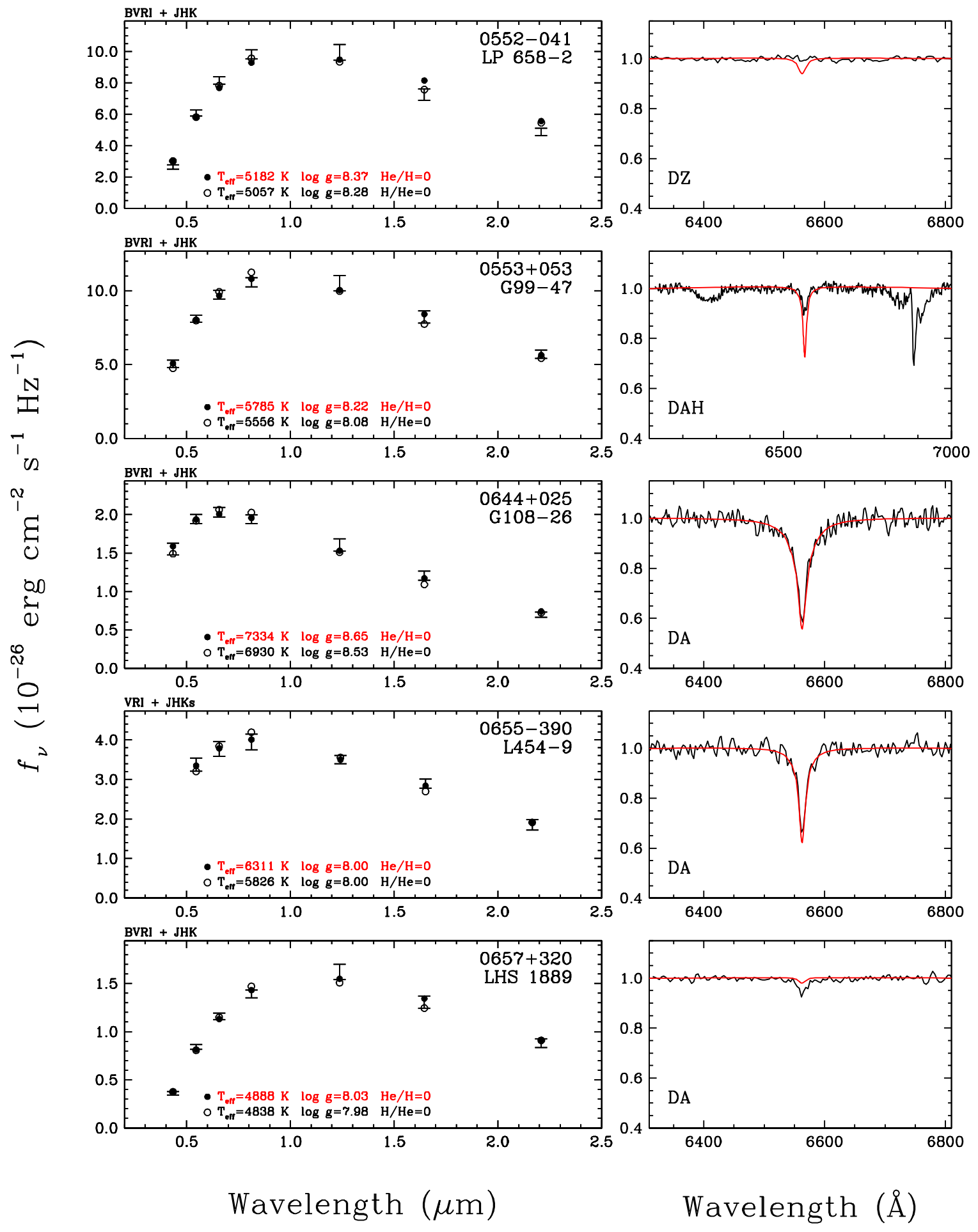

Figure 11g 


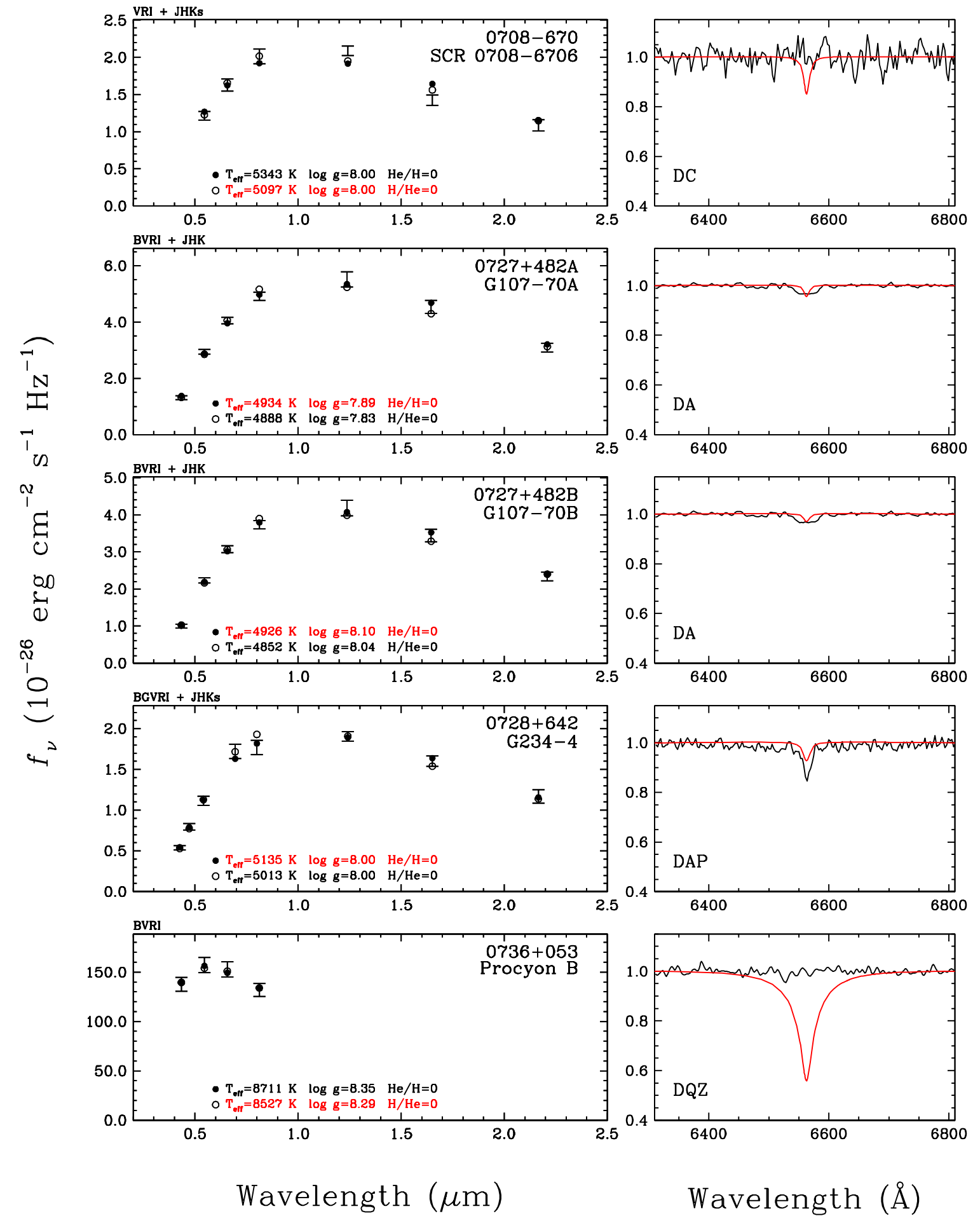

Figure 11h 


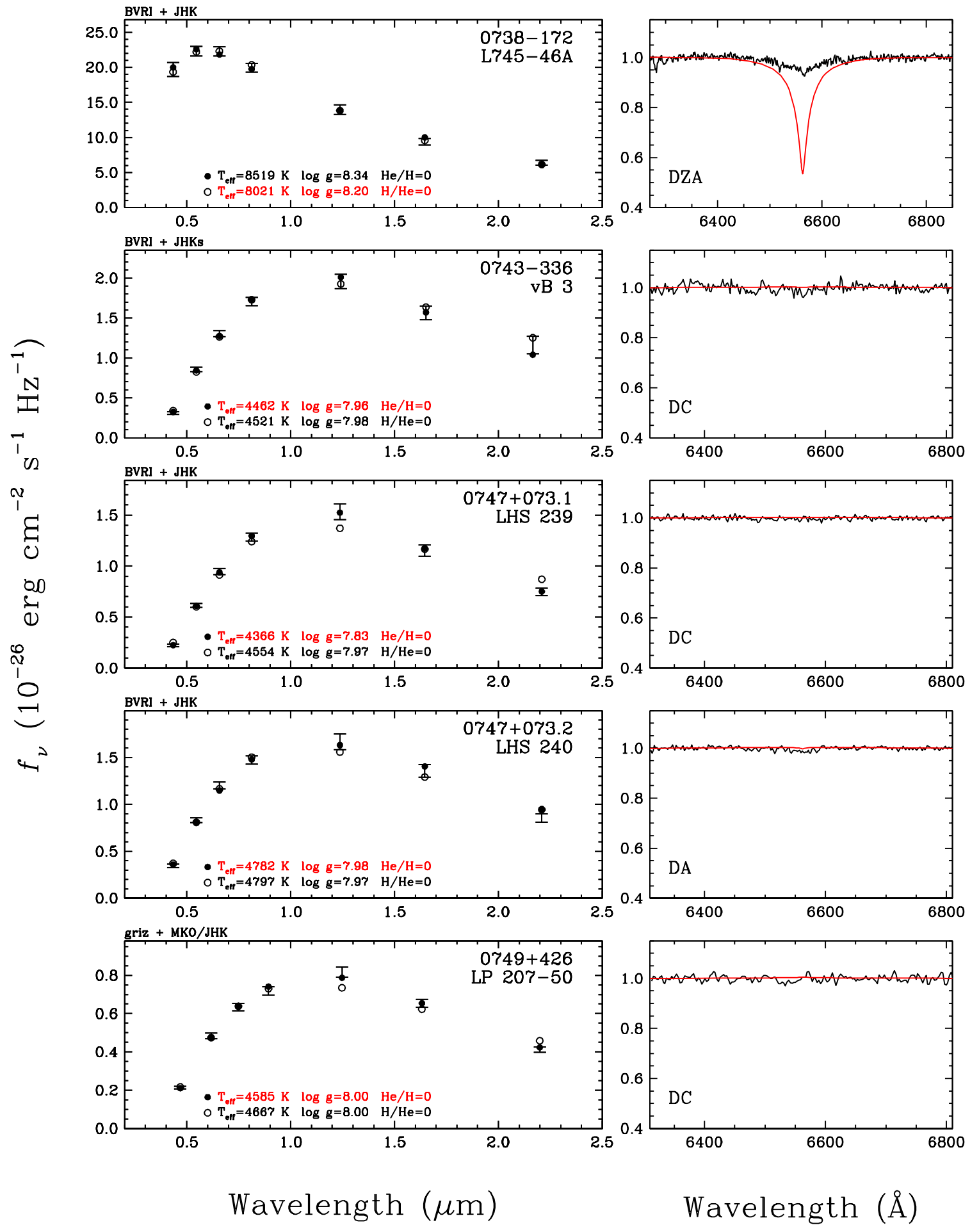

Figure 11. 


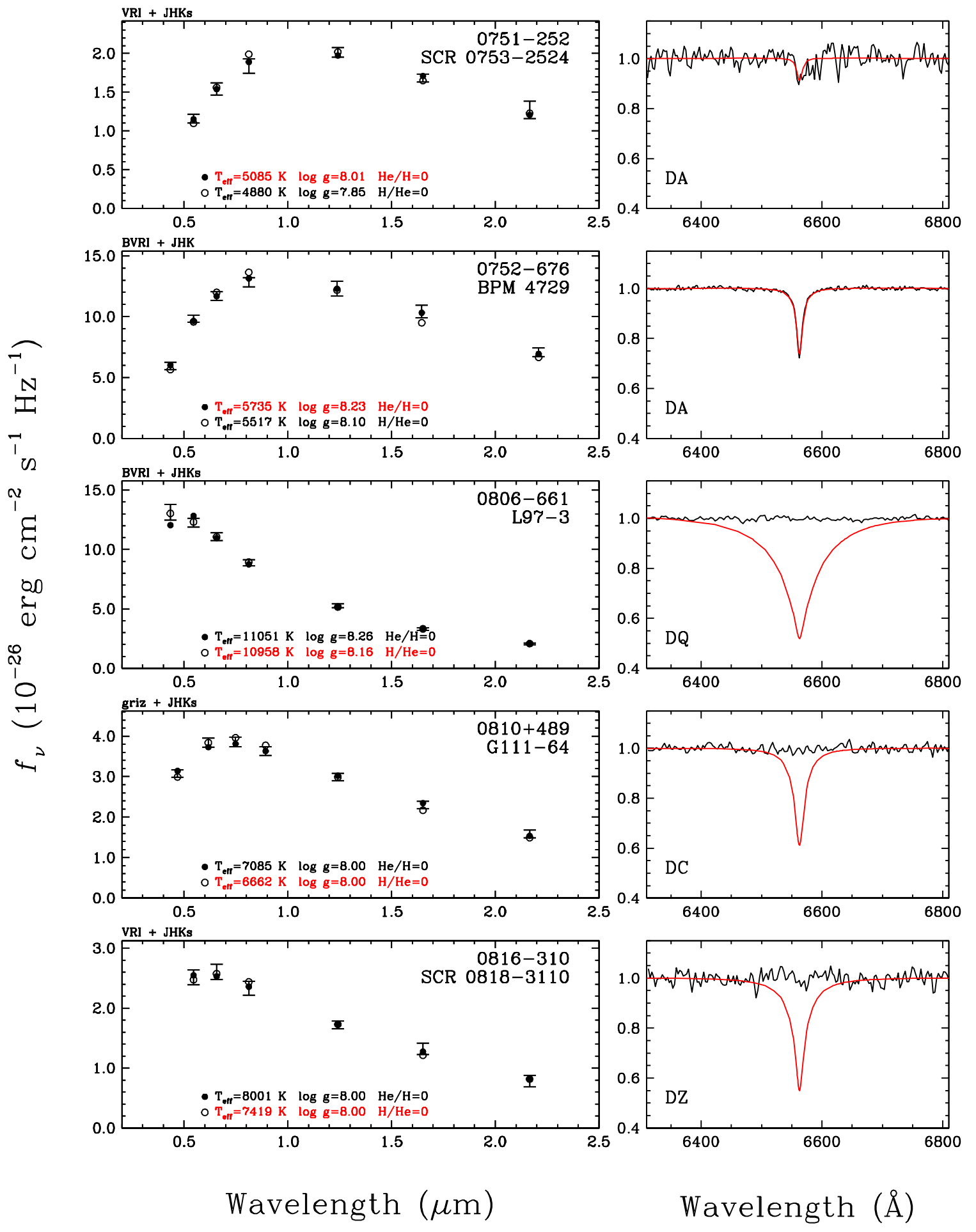

Figure 11] 


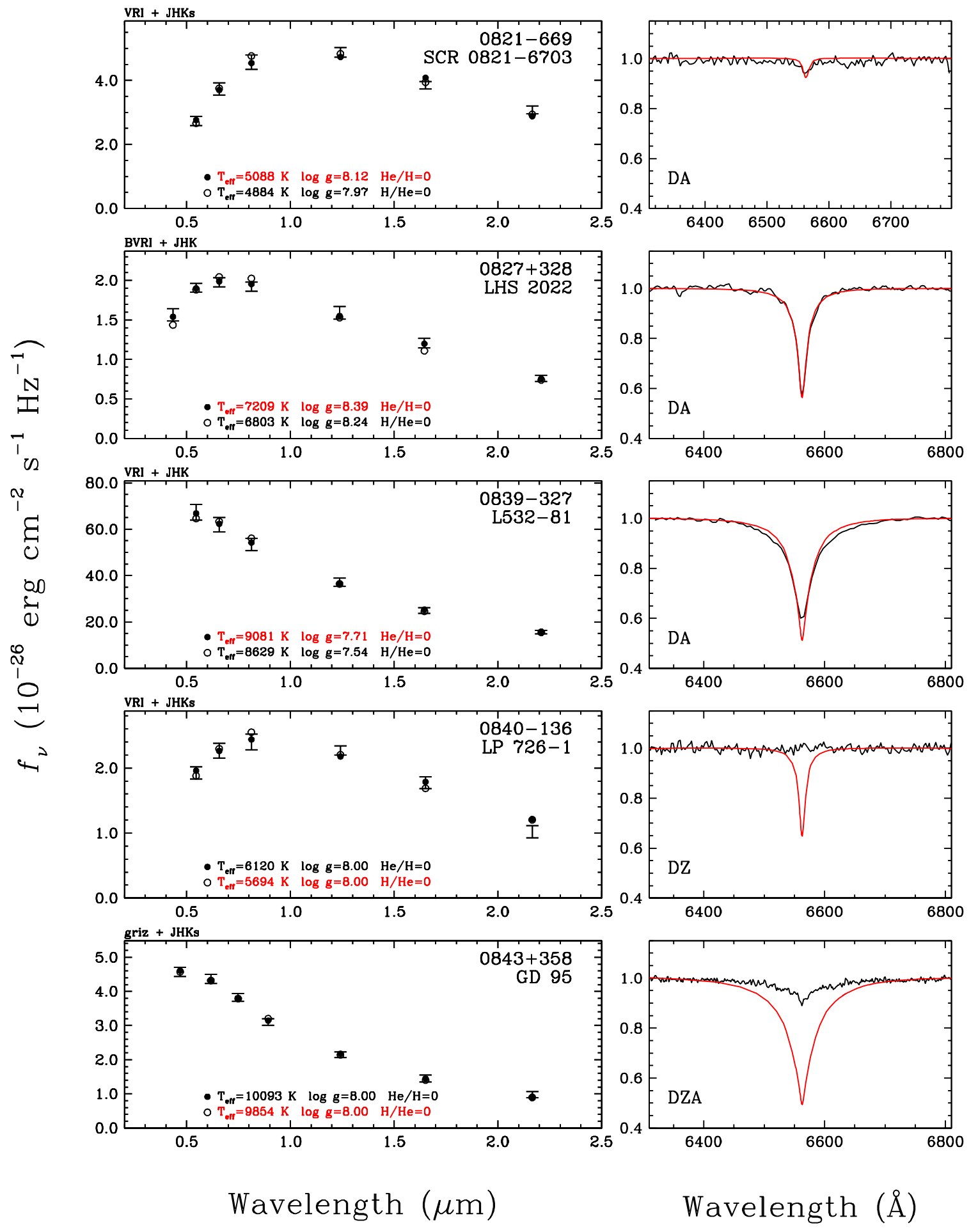

Figure 11k 


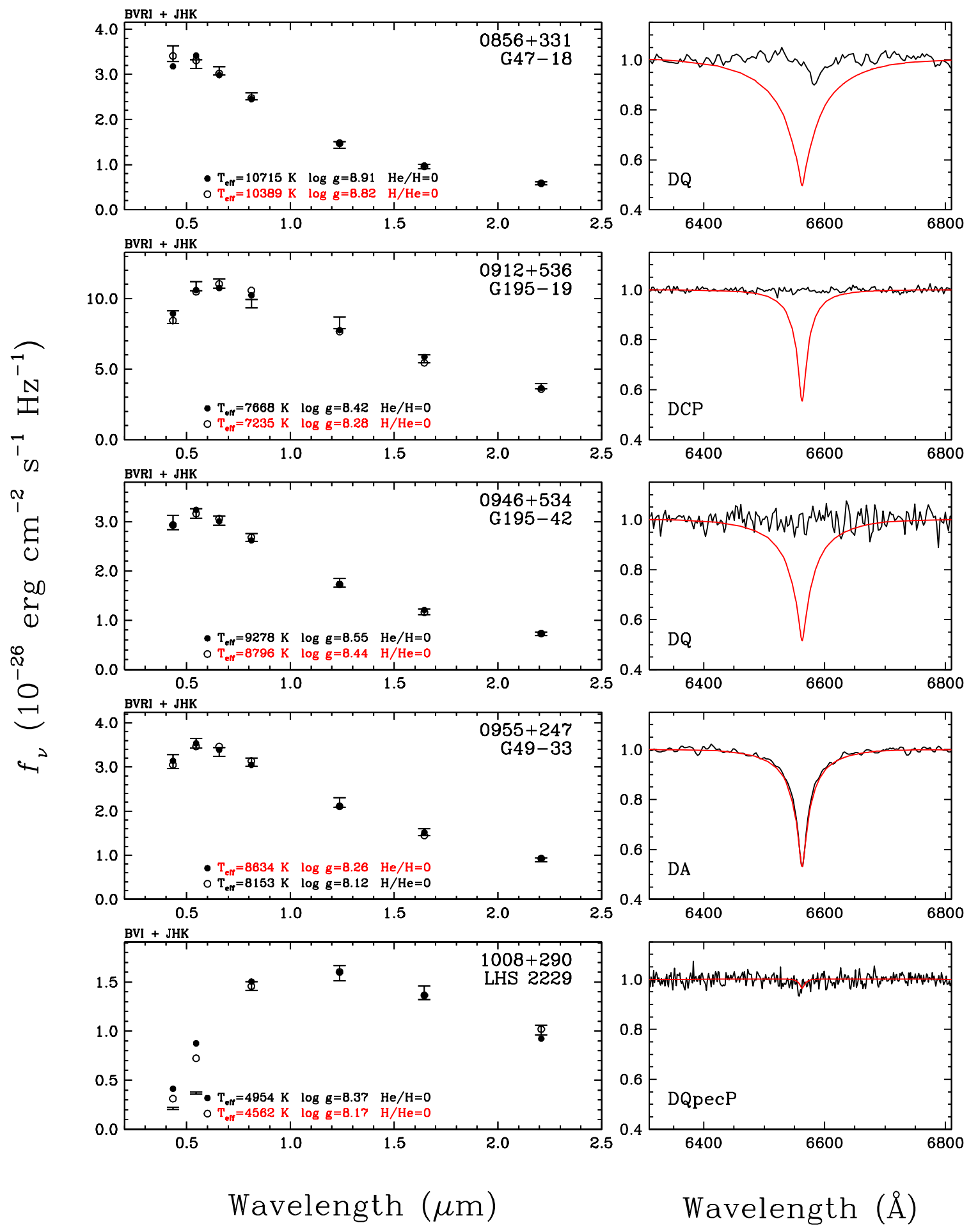

Figure 11. 


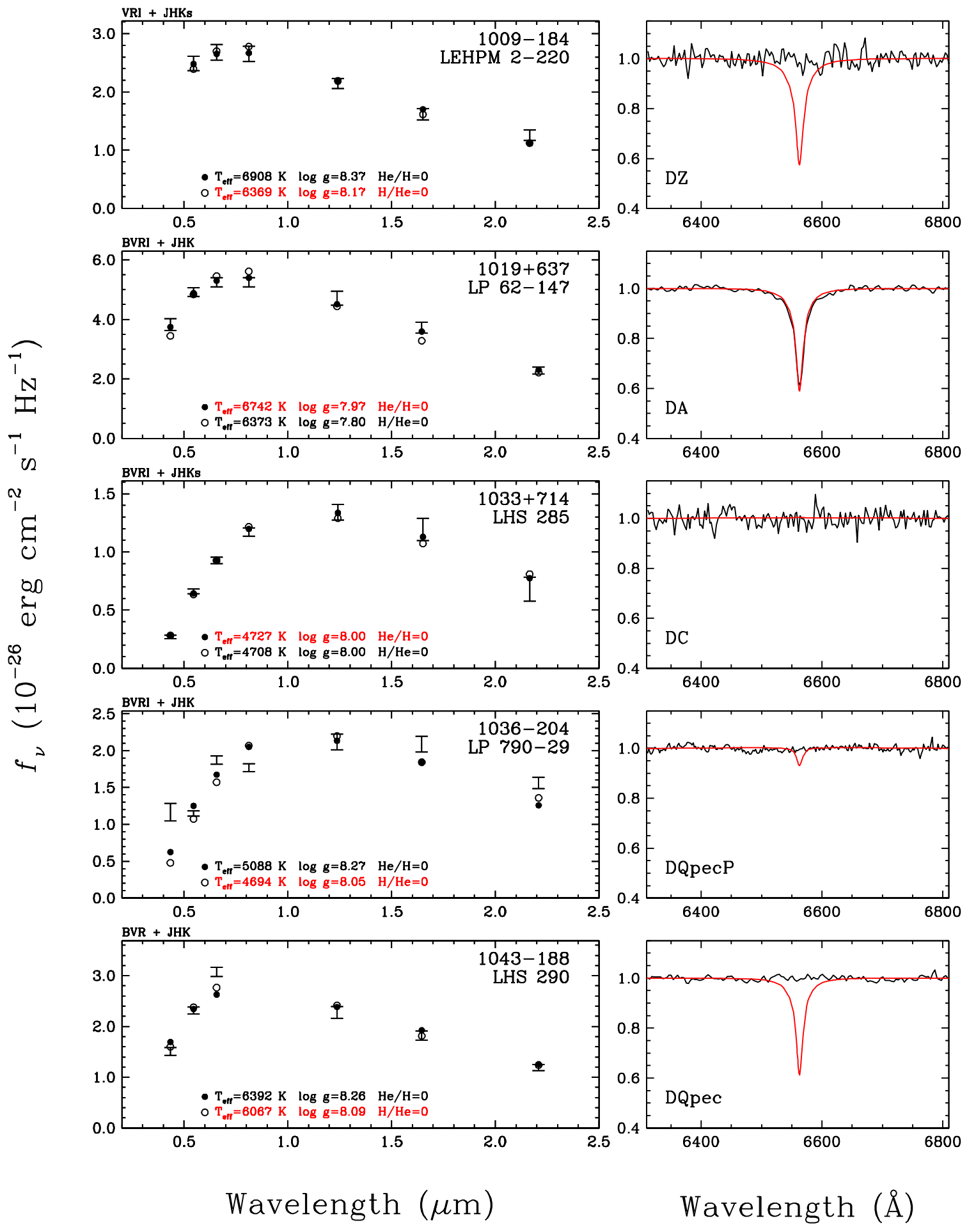

Figure 11m 


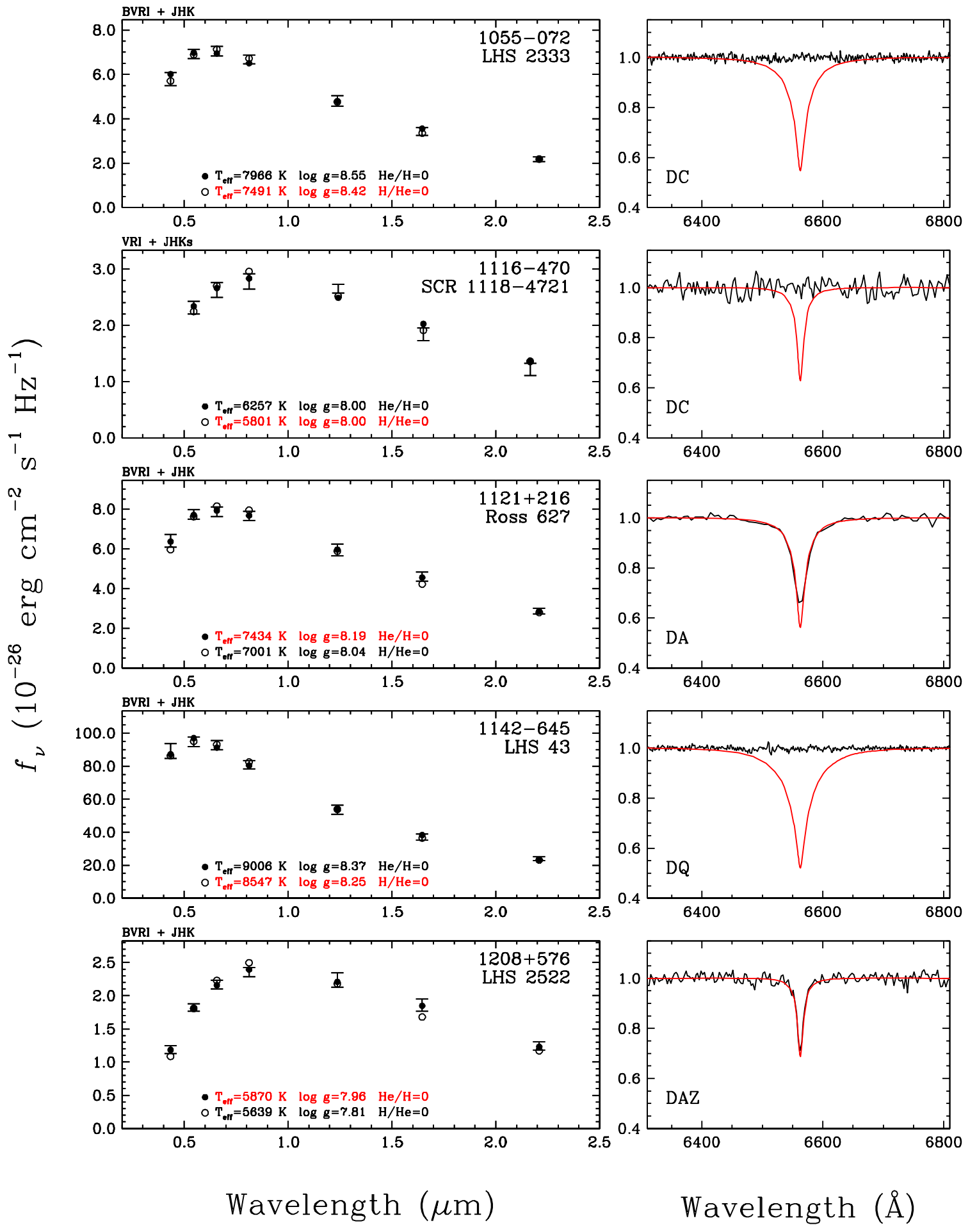

Figure 11n 


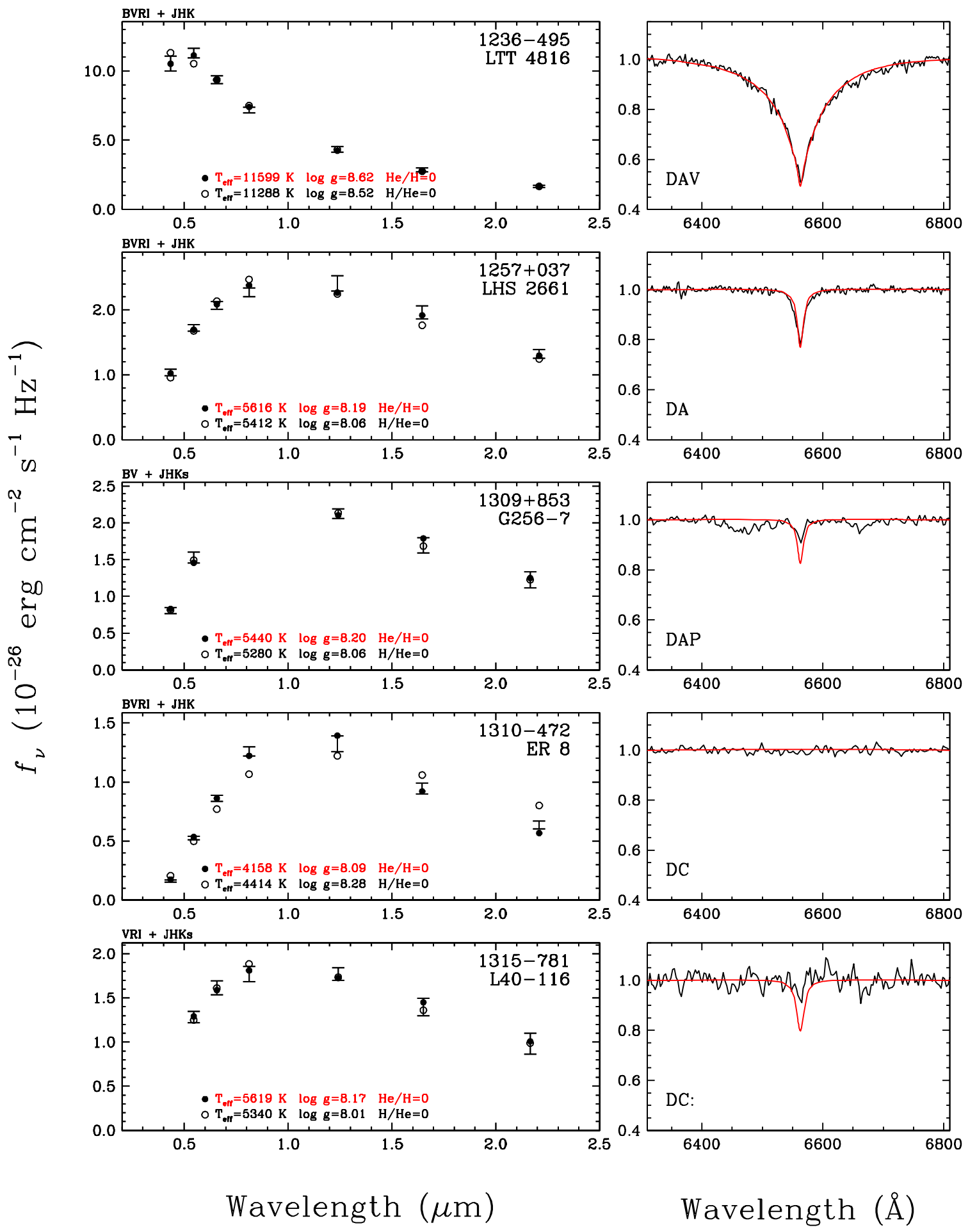

Figure $11 b$ 


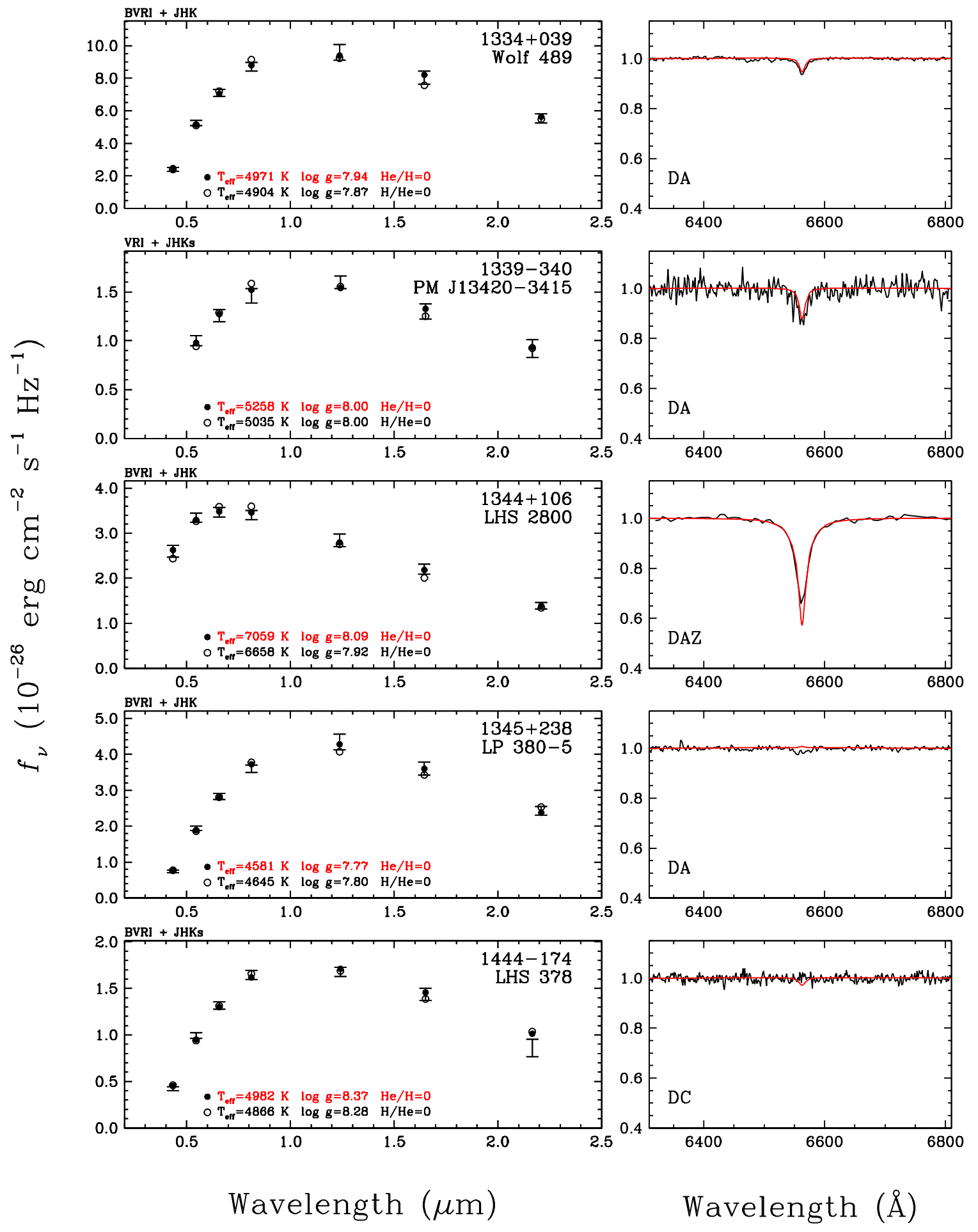

Figure 11p 


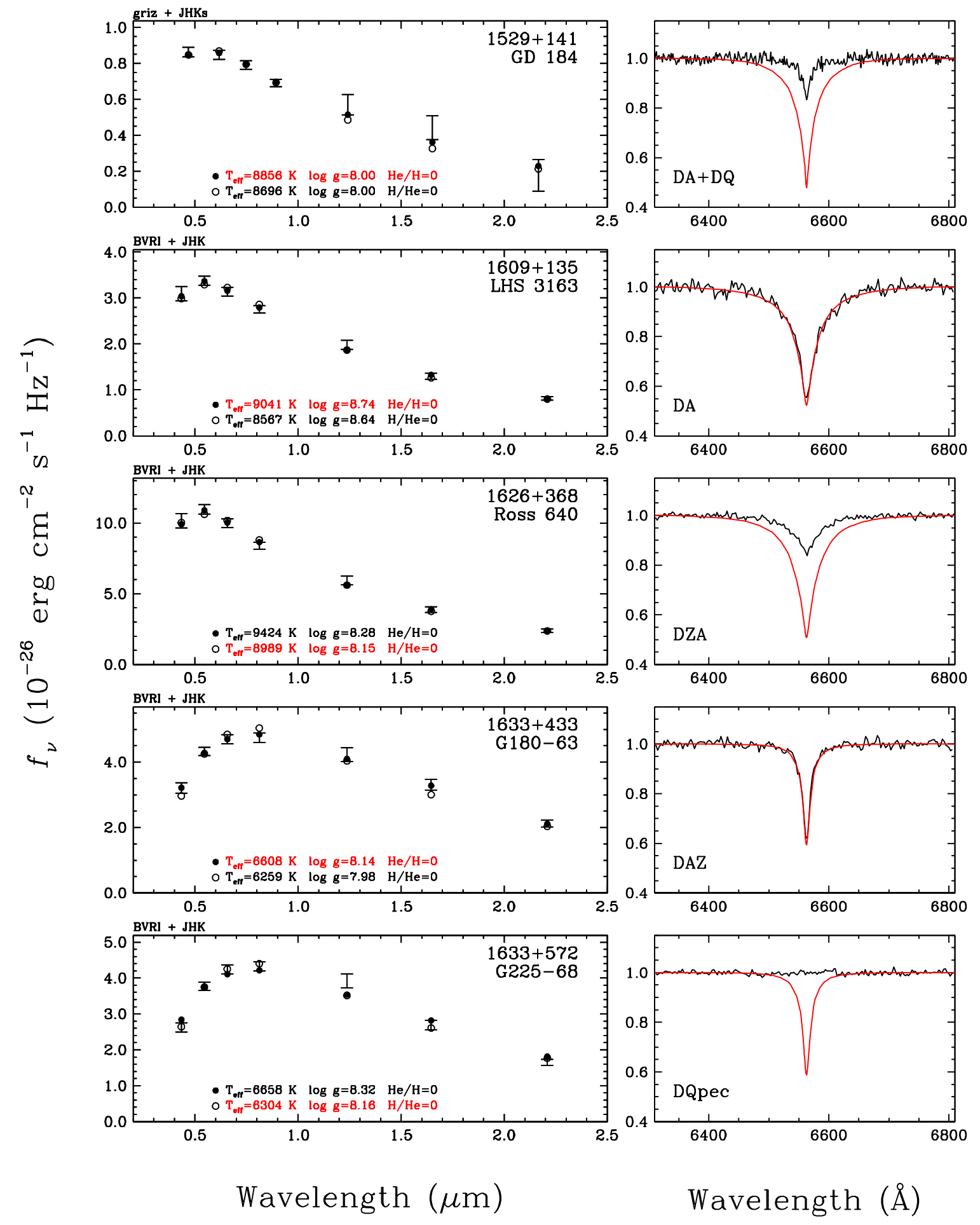

Figure 11 a 


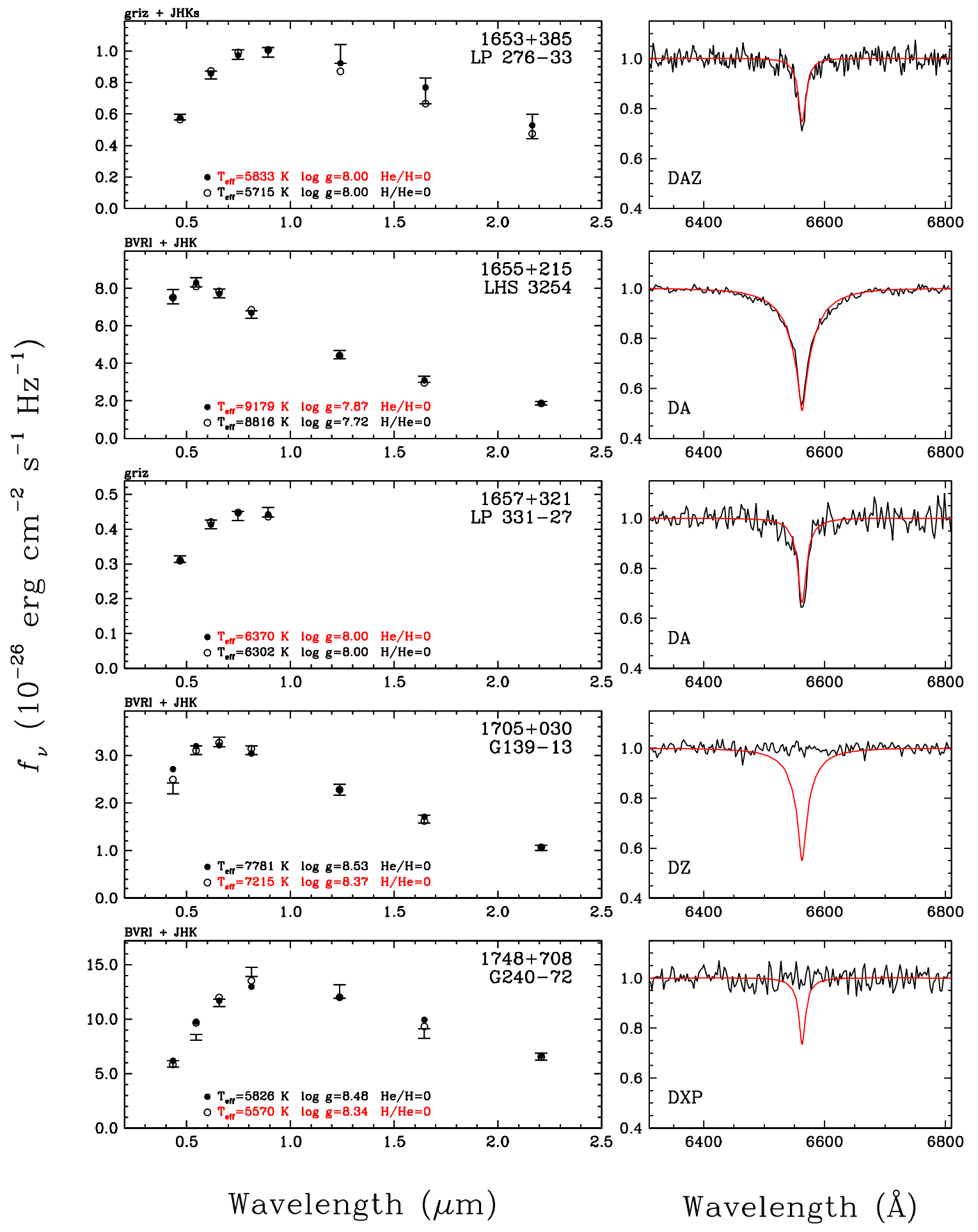

Figure 11r 


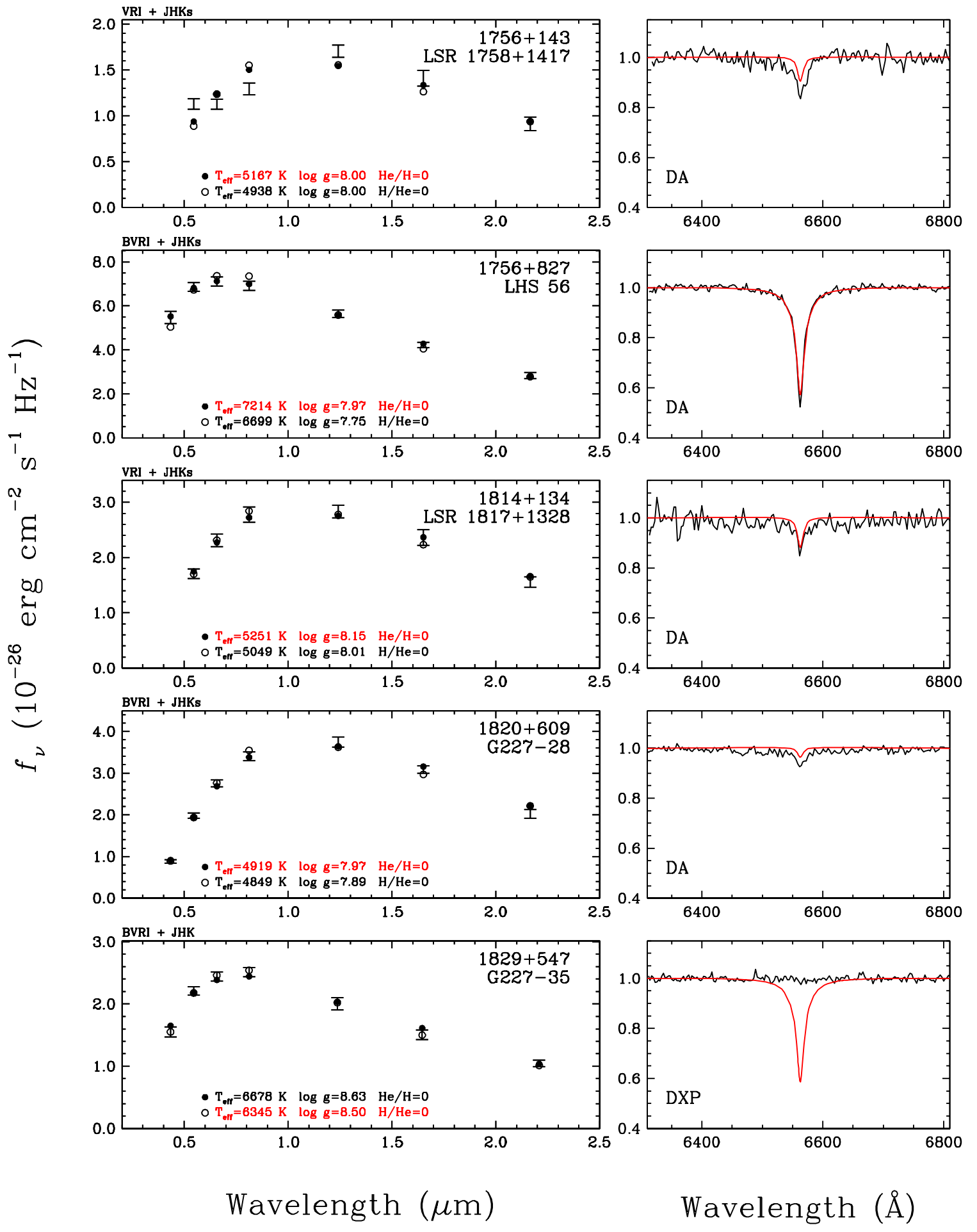

Figure $11 s$ 


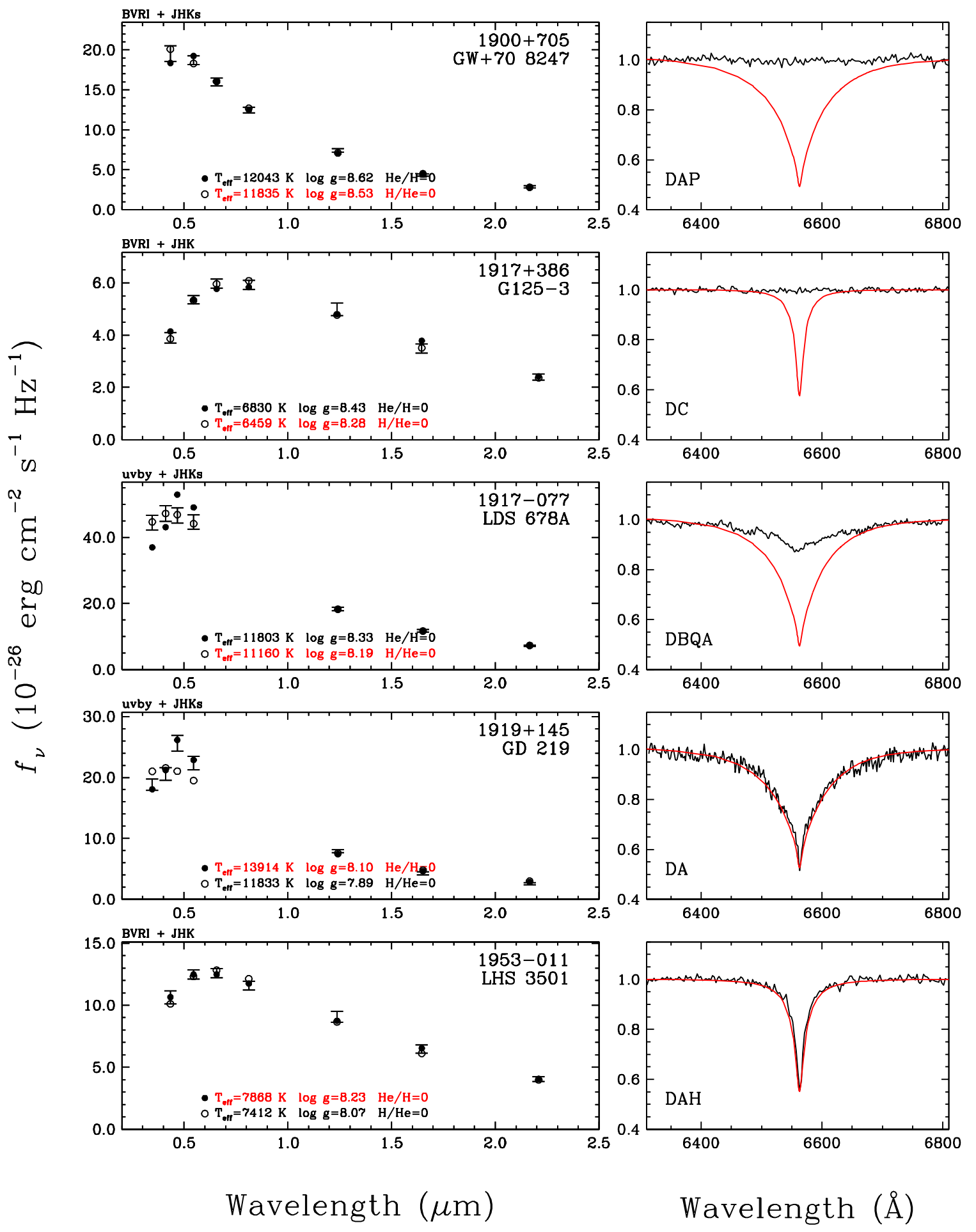

Figure 11t 


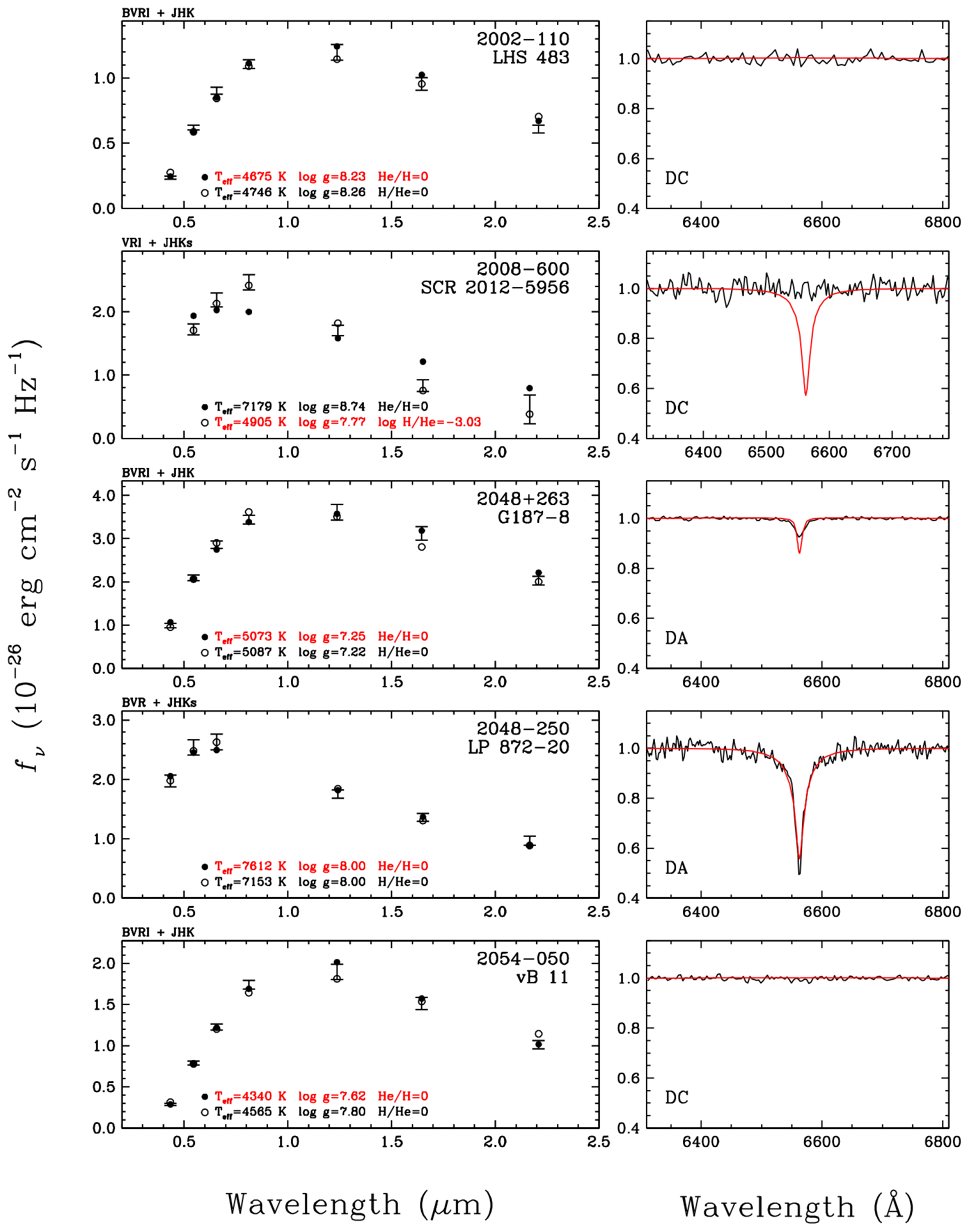

Figure 11] 


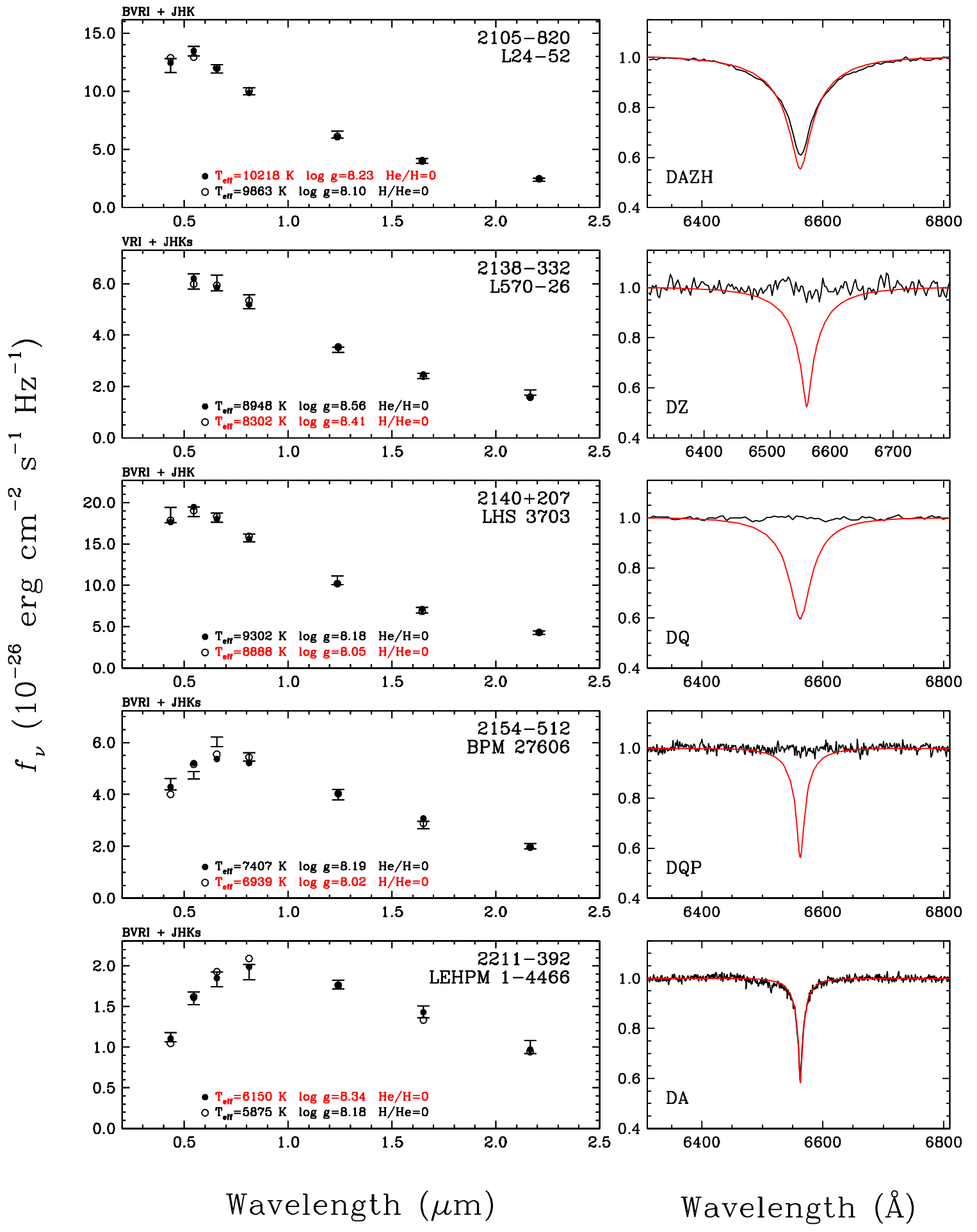

Figure 11v 


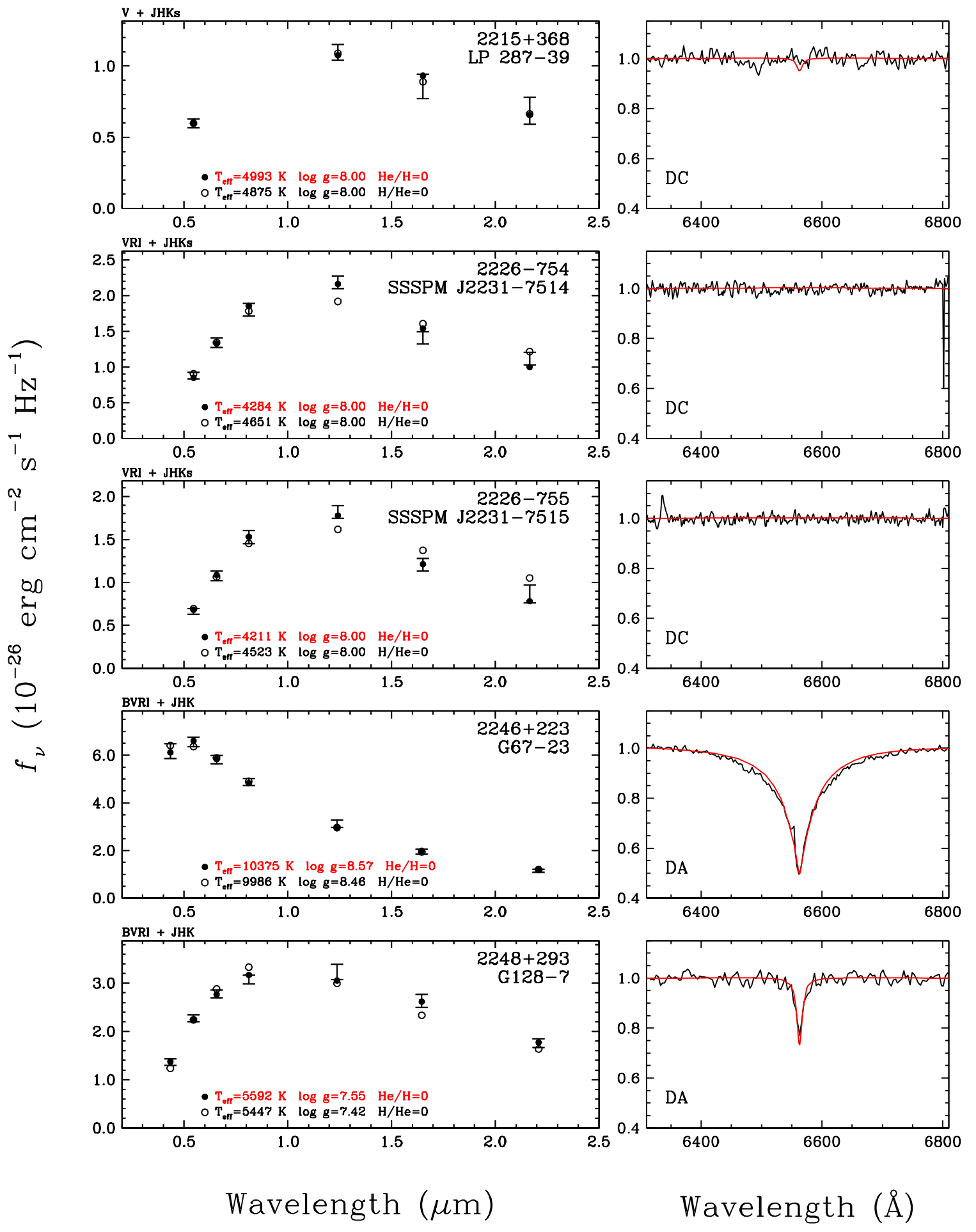

Figure $11 \mathrm{w}$ 


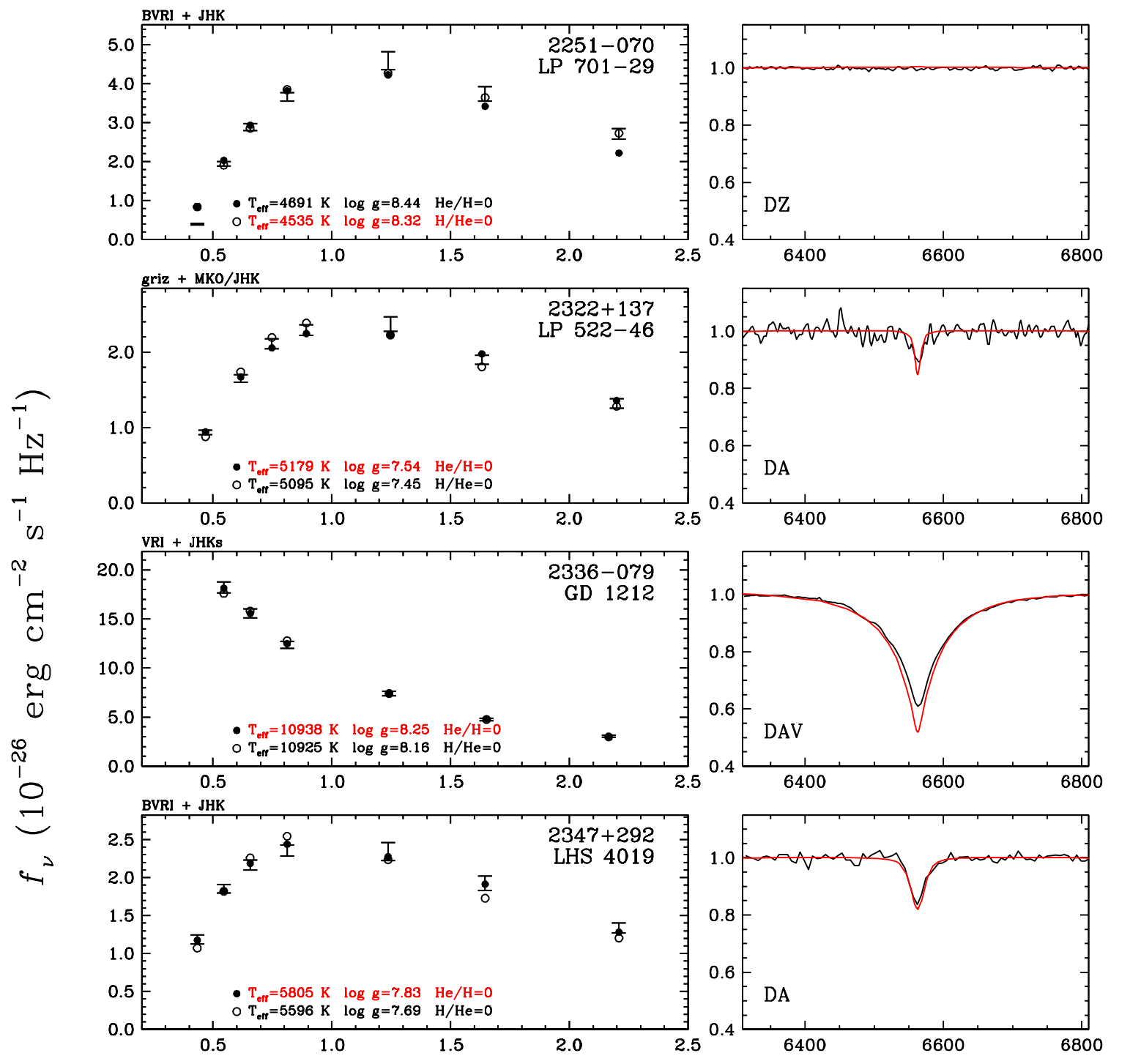

Wavelength $(\mu \mathrm{m})$

Wavelength $(\AA)$

Figure 11x 


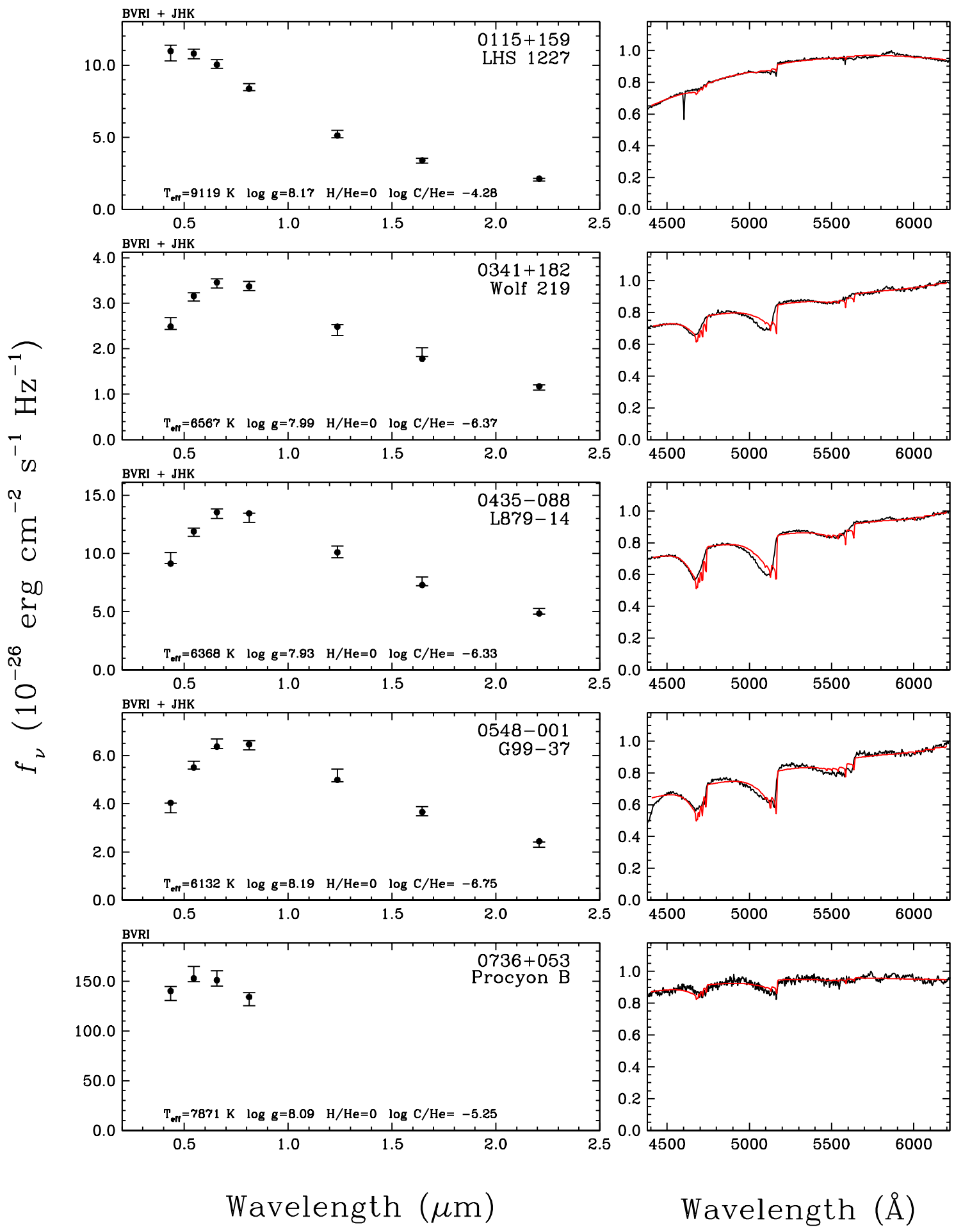

Figure $12 a$ 


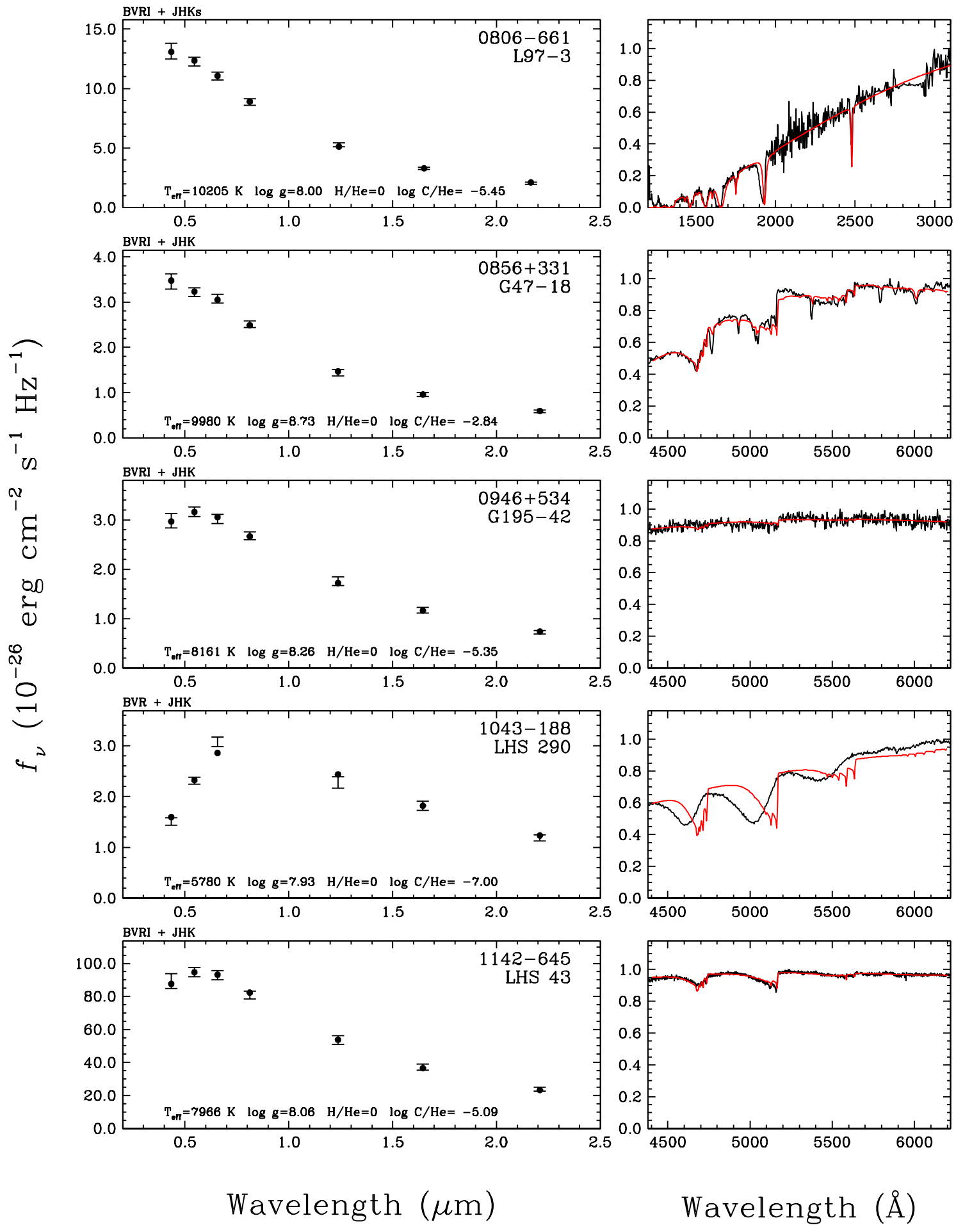

Figure 12b 


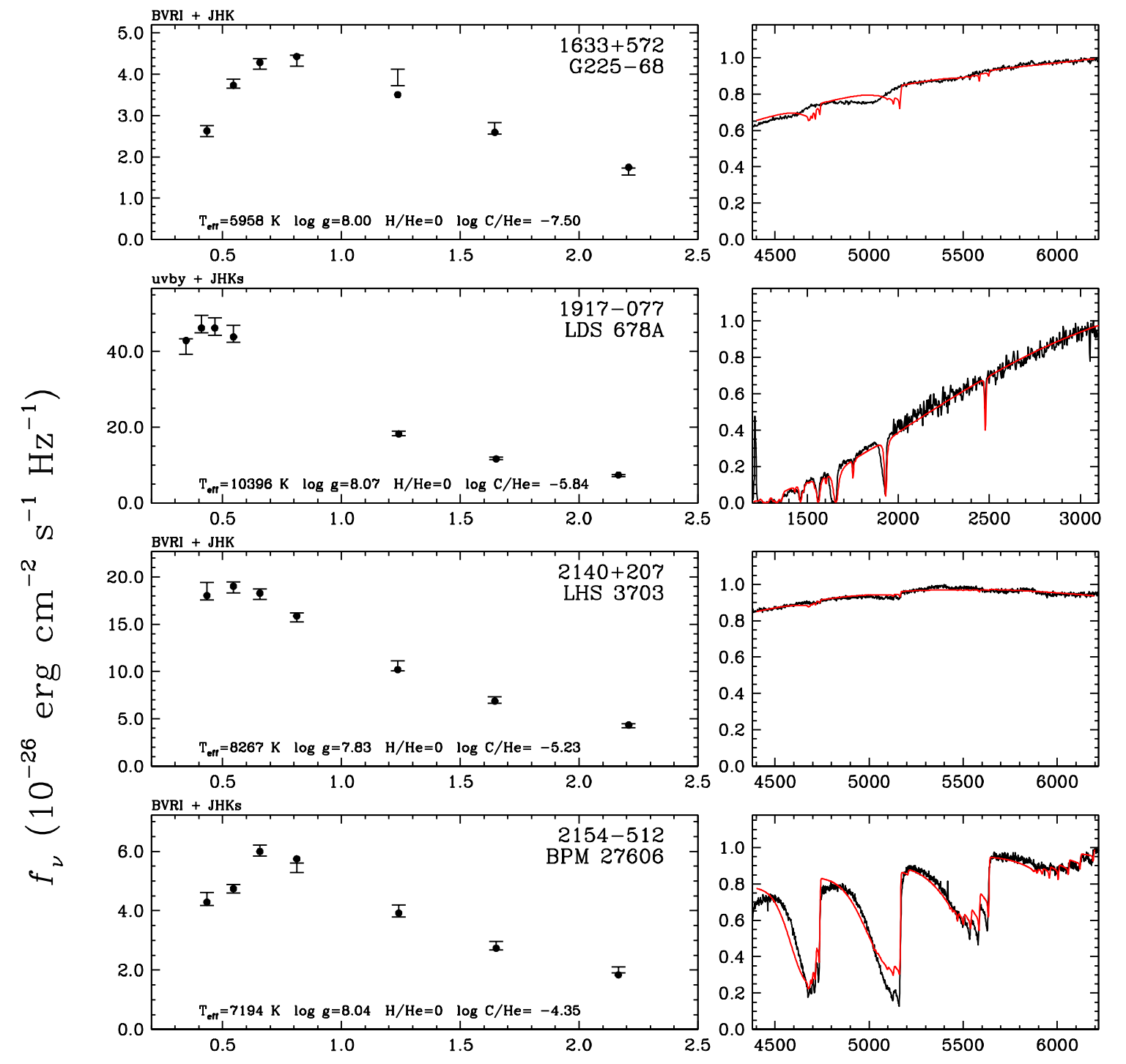

Wavelength $(\mu \mathrm{m})$

Wavelength $(\AA)$

Figure 12c 

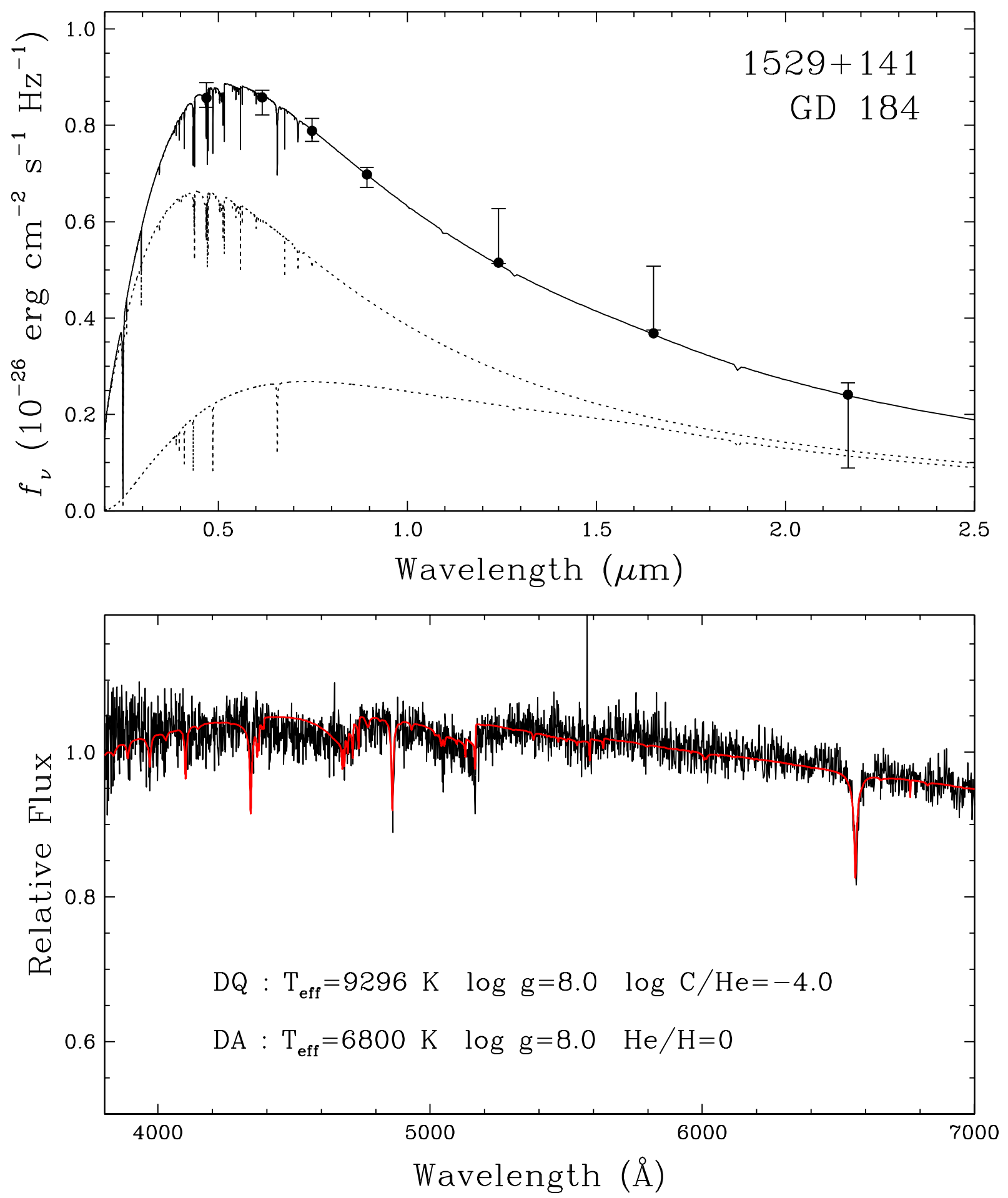

Figure 13 


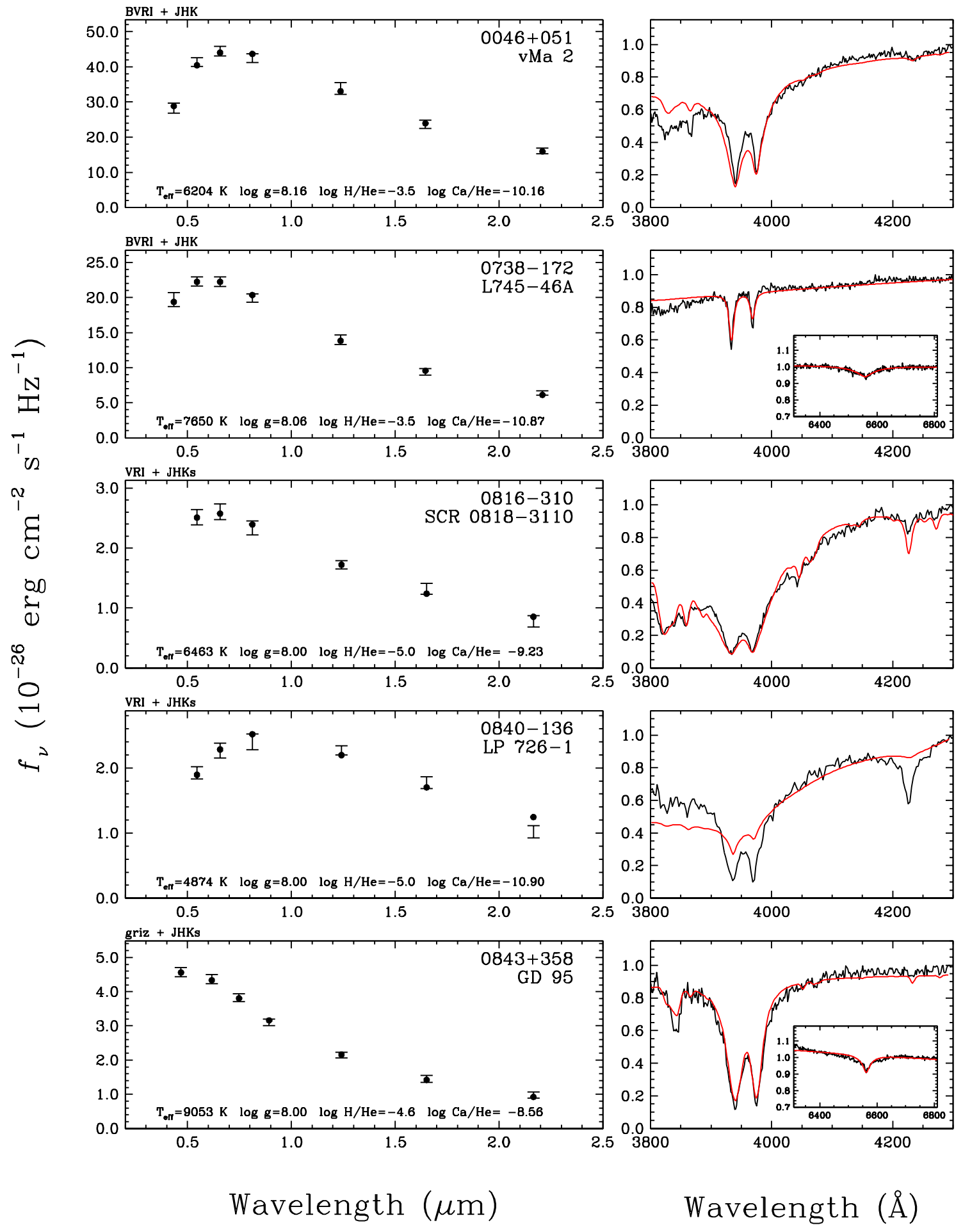

Figure 14a 


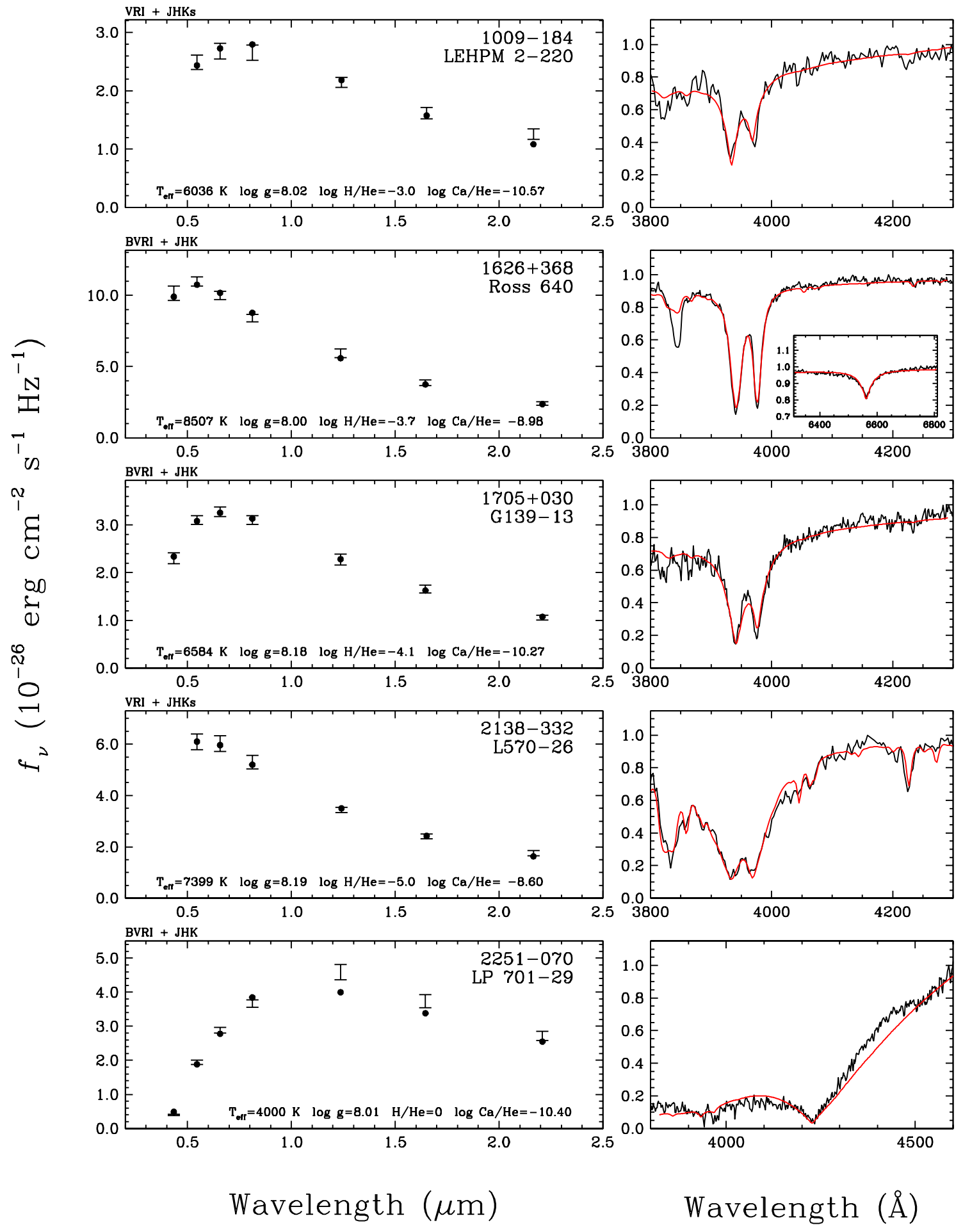

Figure 14b 


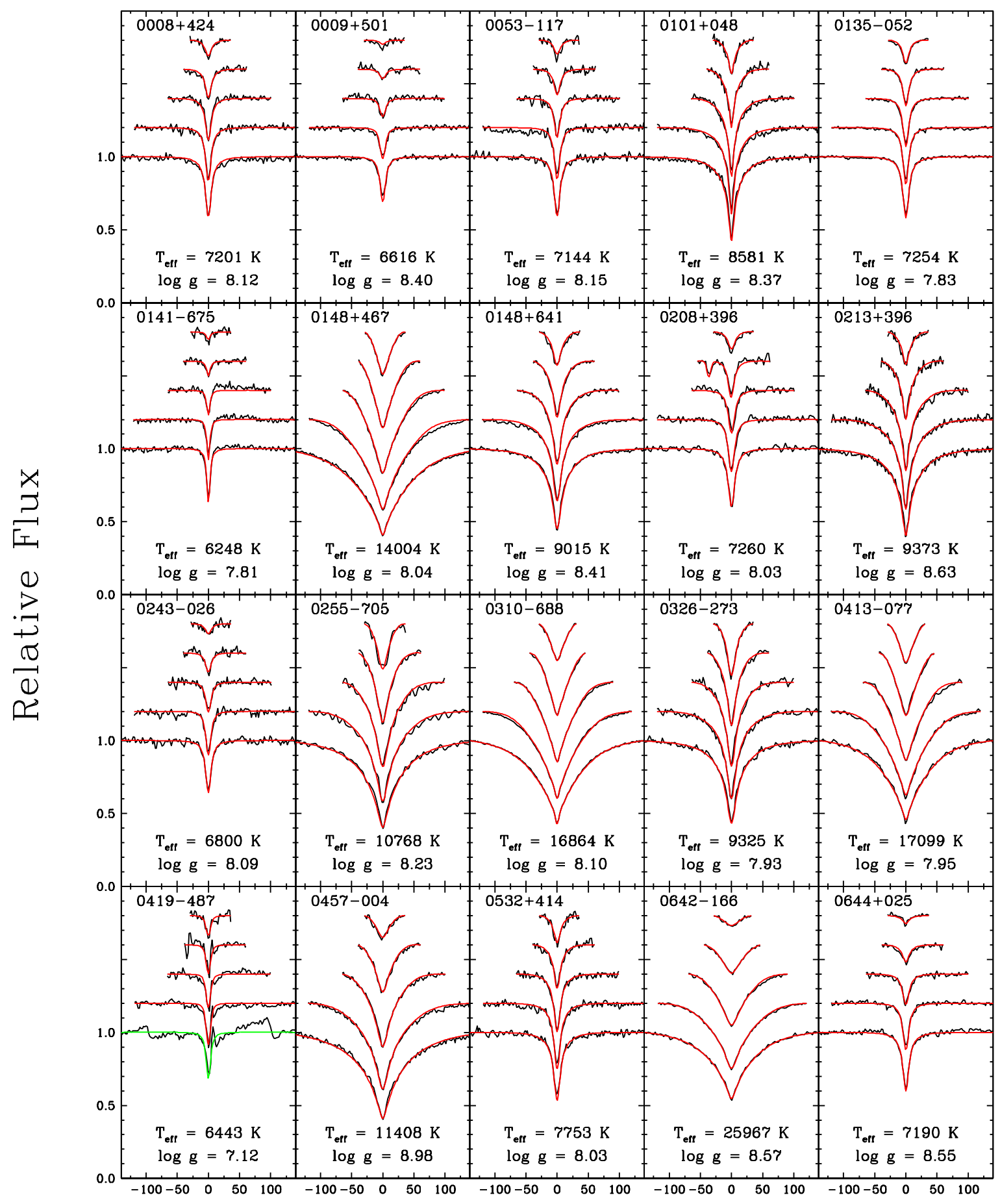

$\Delta \lambda(\AA)$

Figure 15 


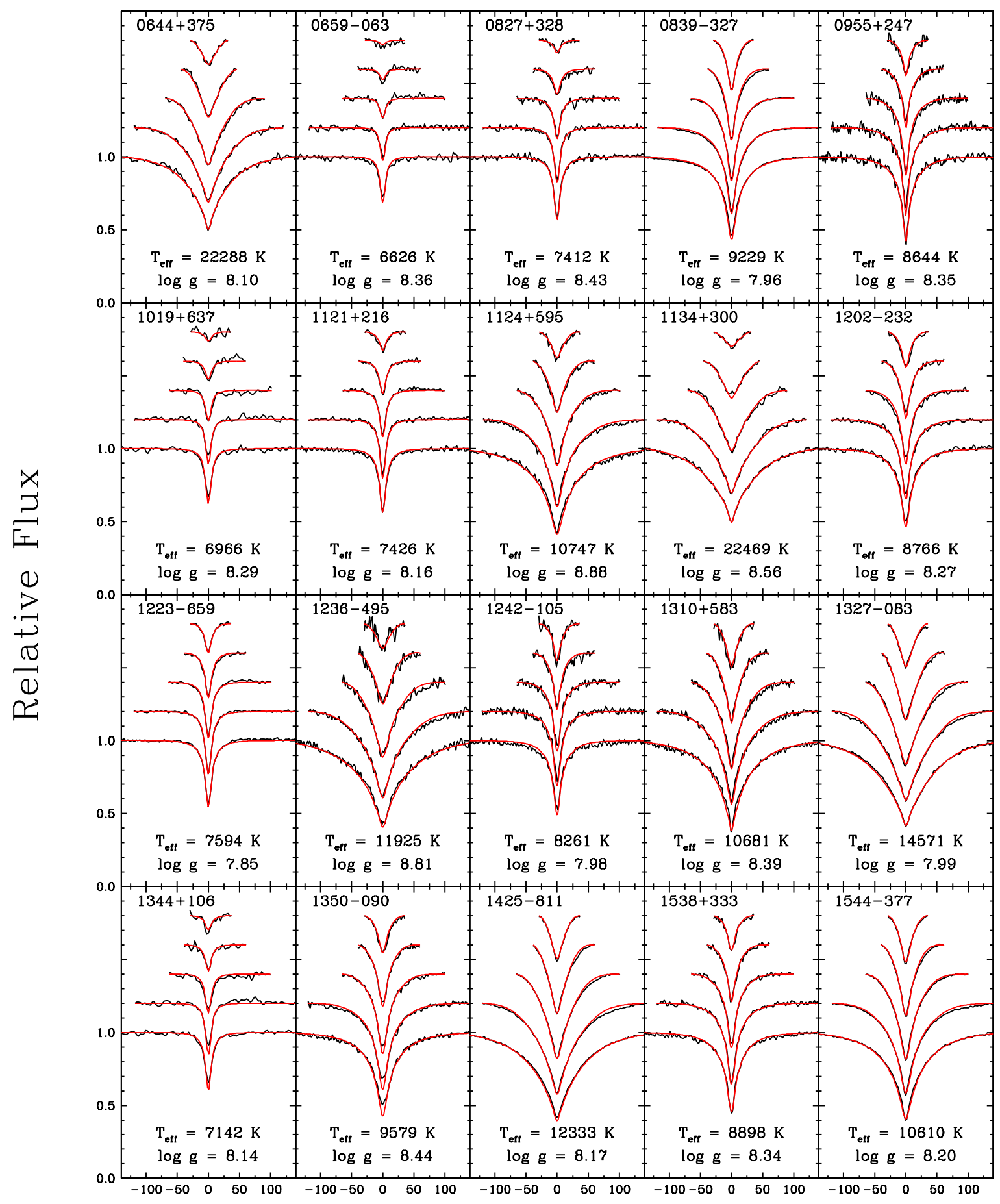

$\Delta \lambda(\AA)$

Figure 15b 


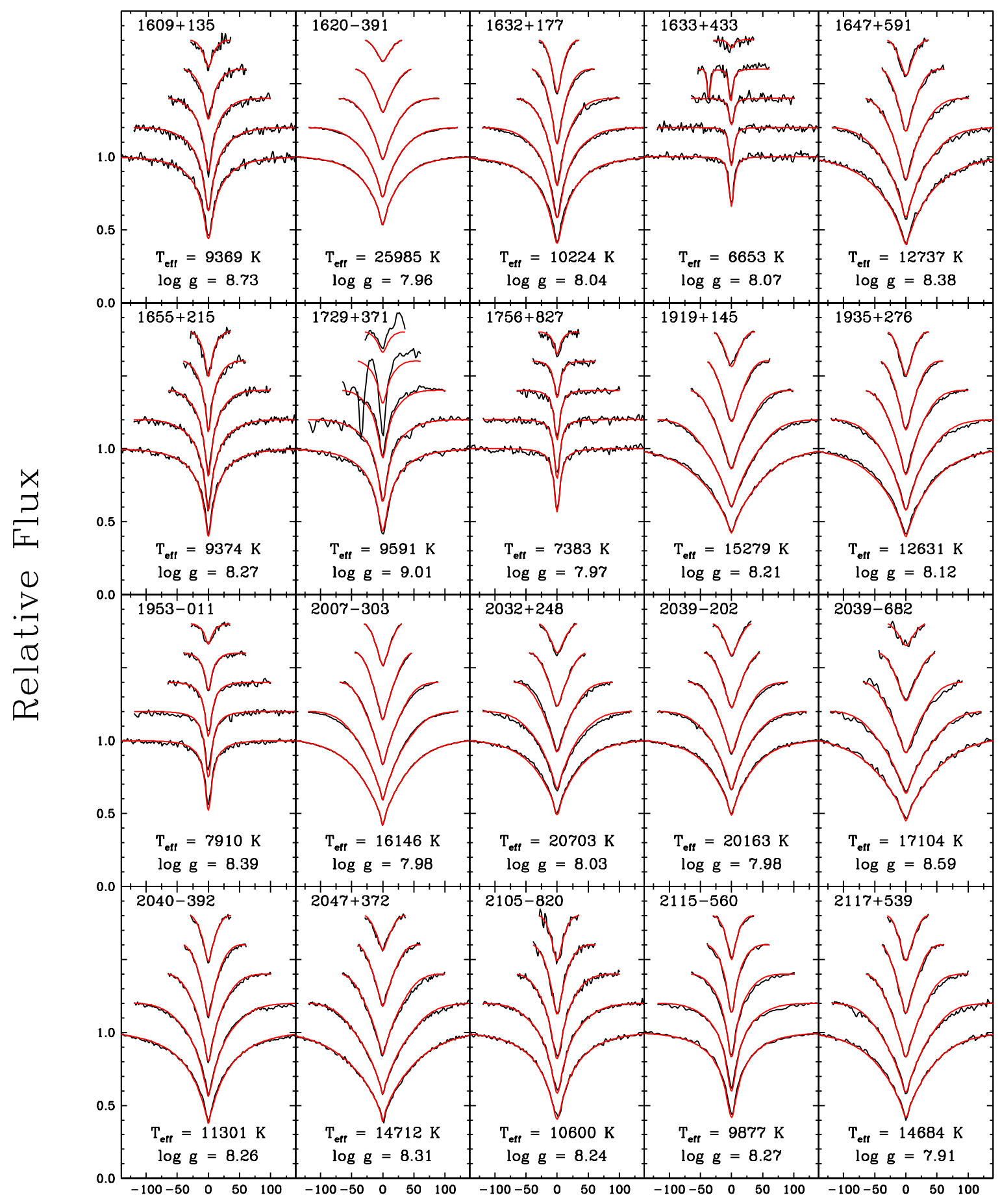

$\Delta \lambda(\AA)$

Figure 15. 


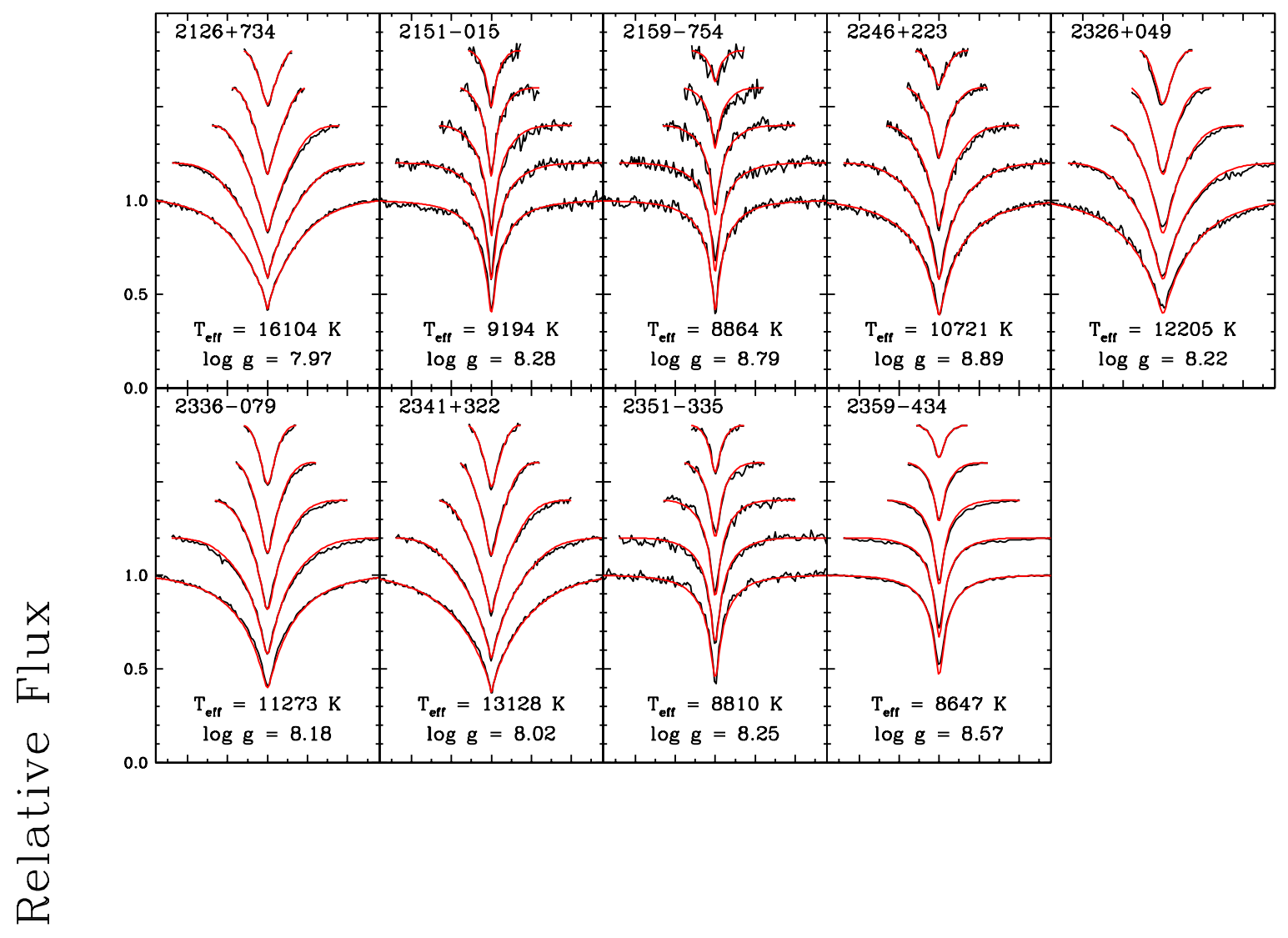

$\Delta \lambda(\AA)$

Figure 15d 


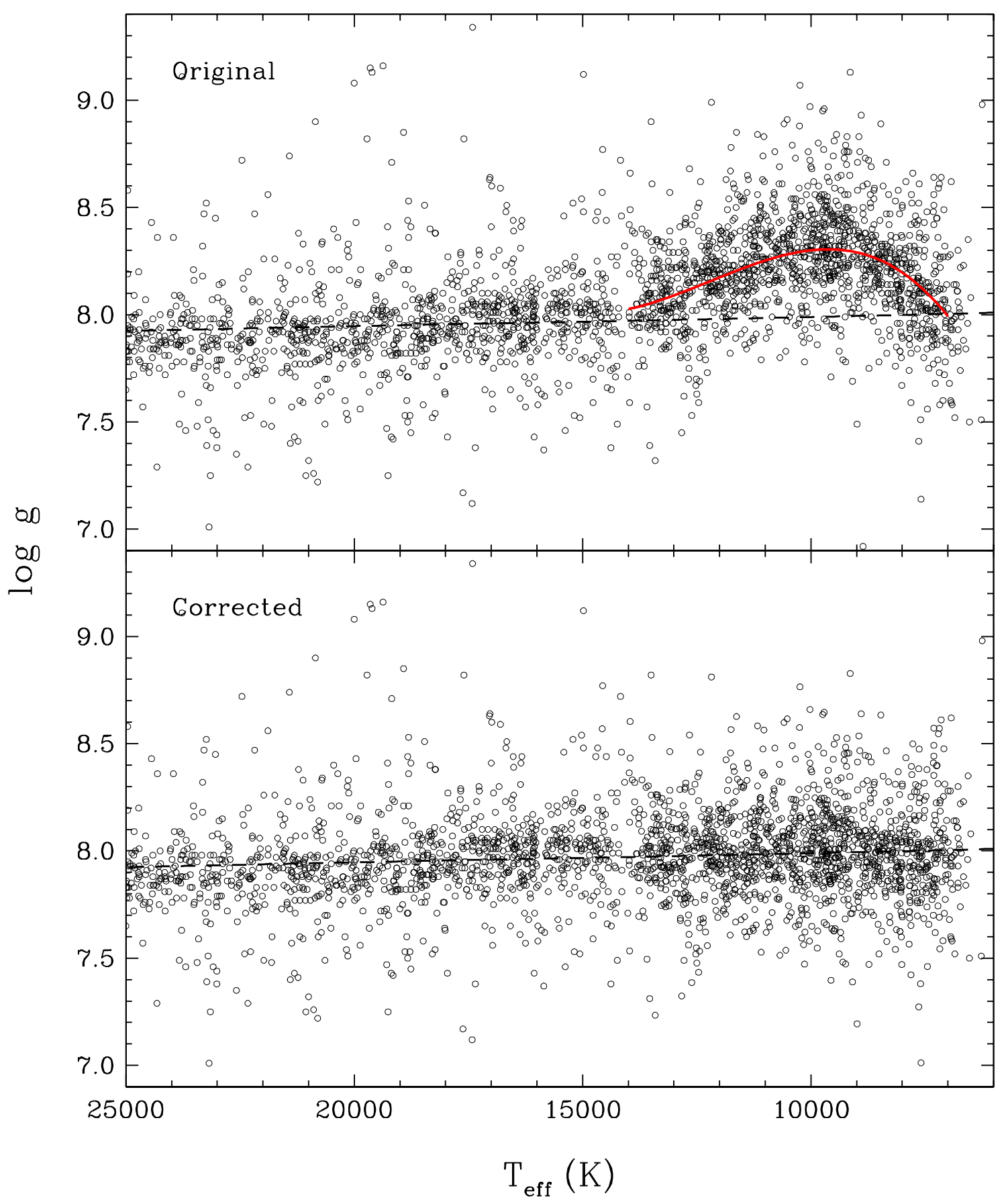

Figure 16 

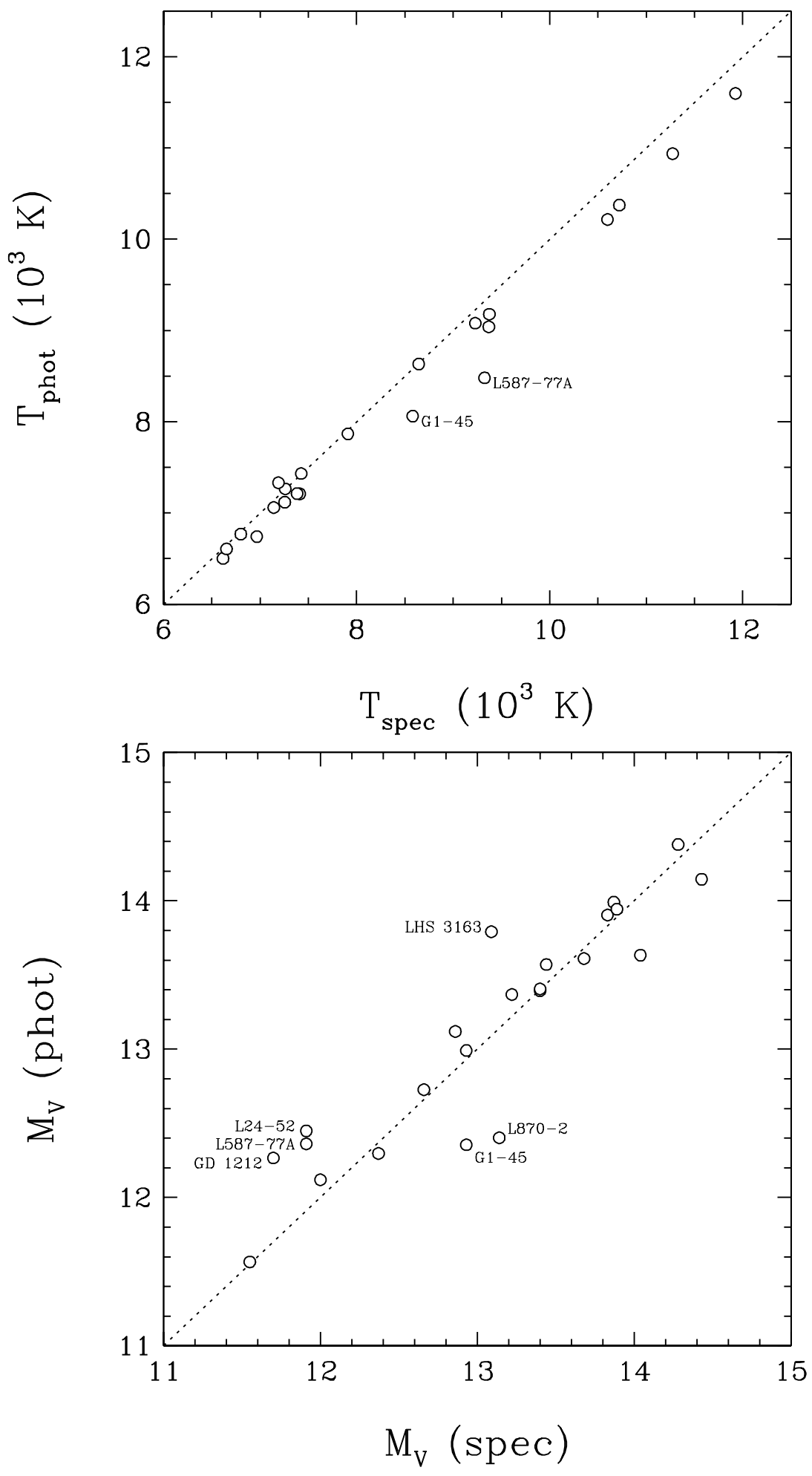

Figure 17 


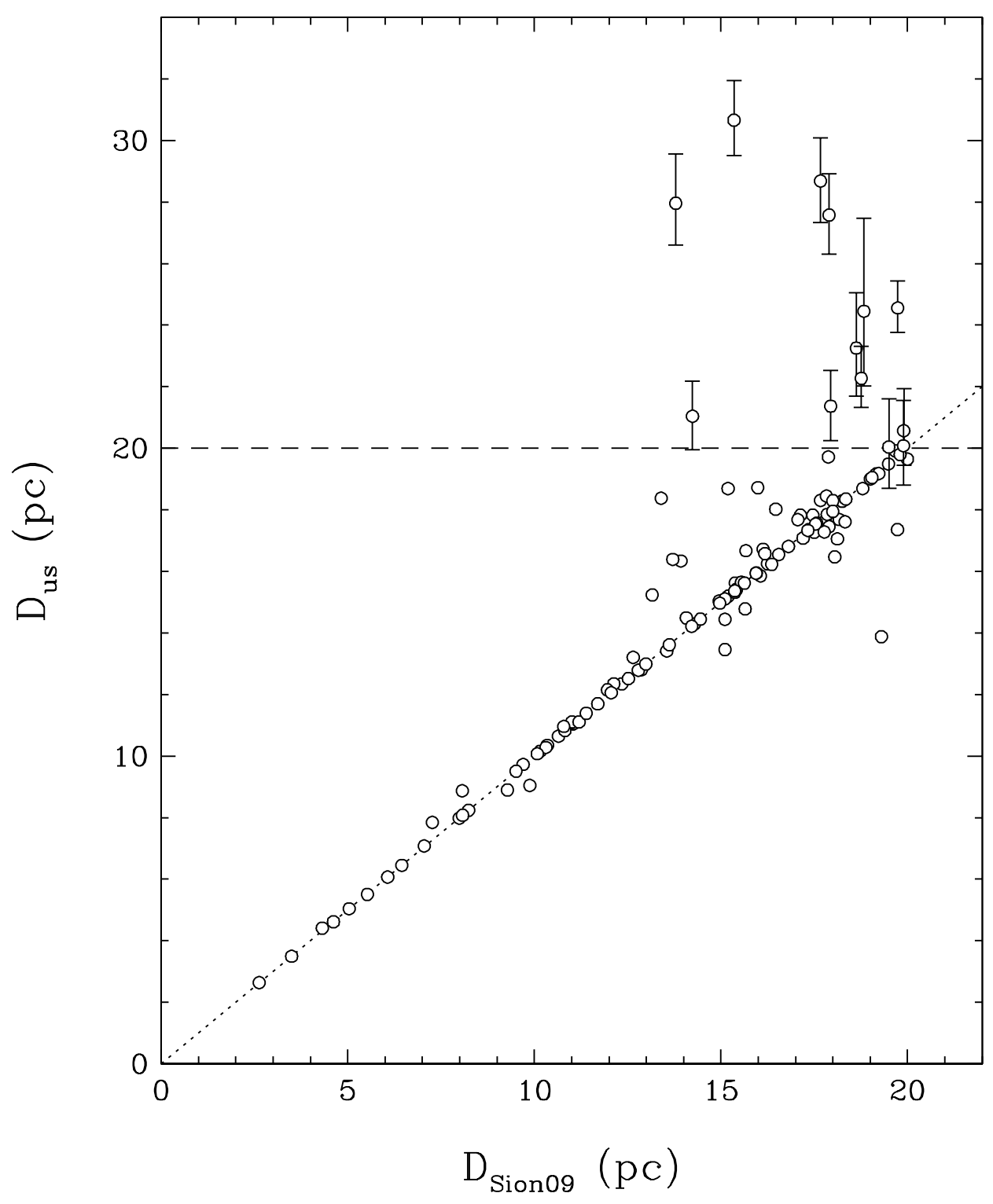

Figure 18 


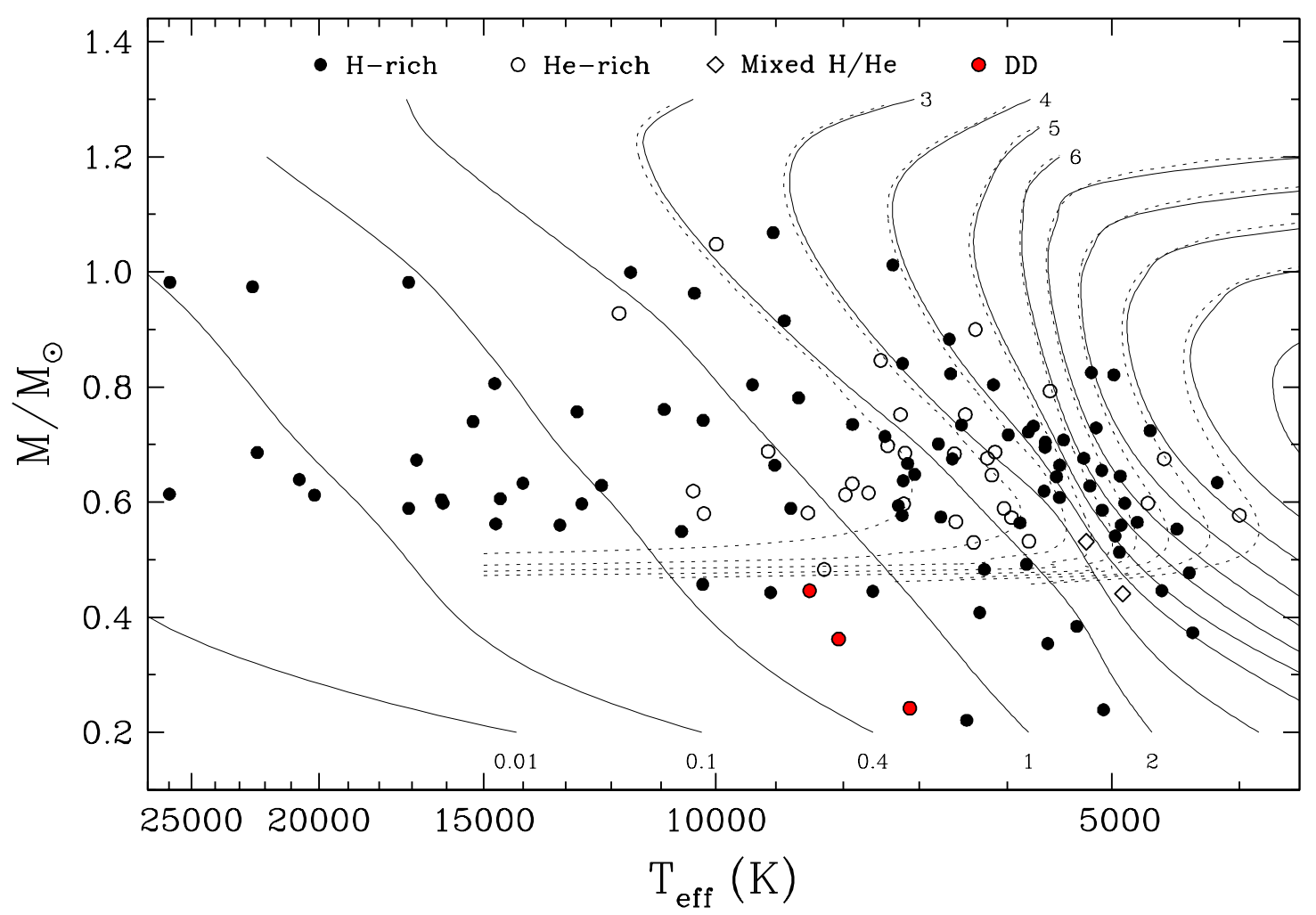

Figure 19 

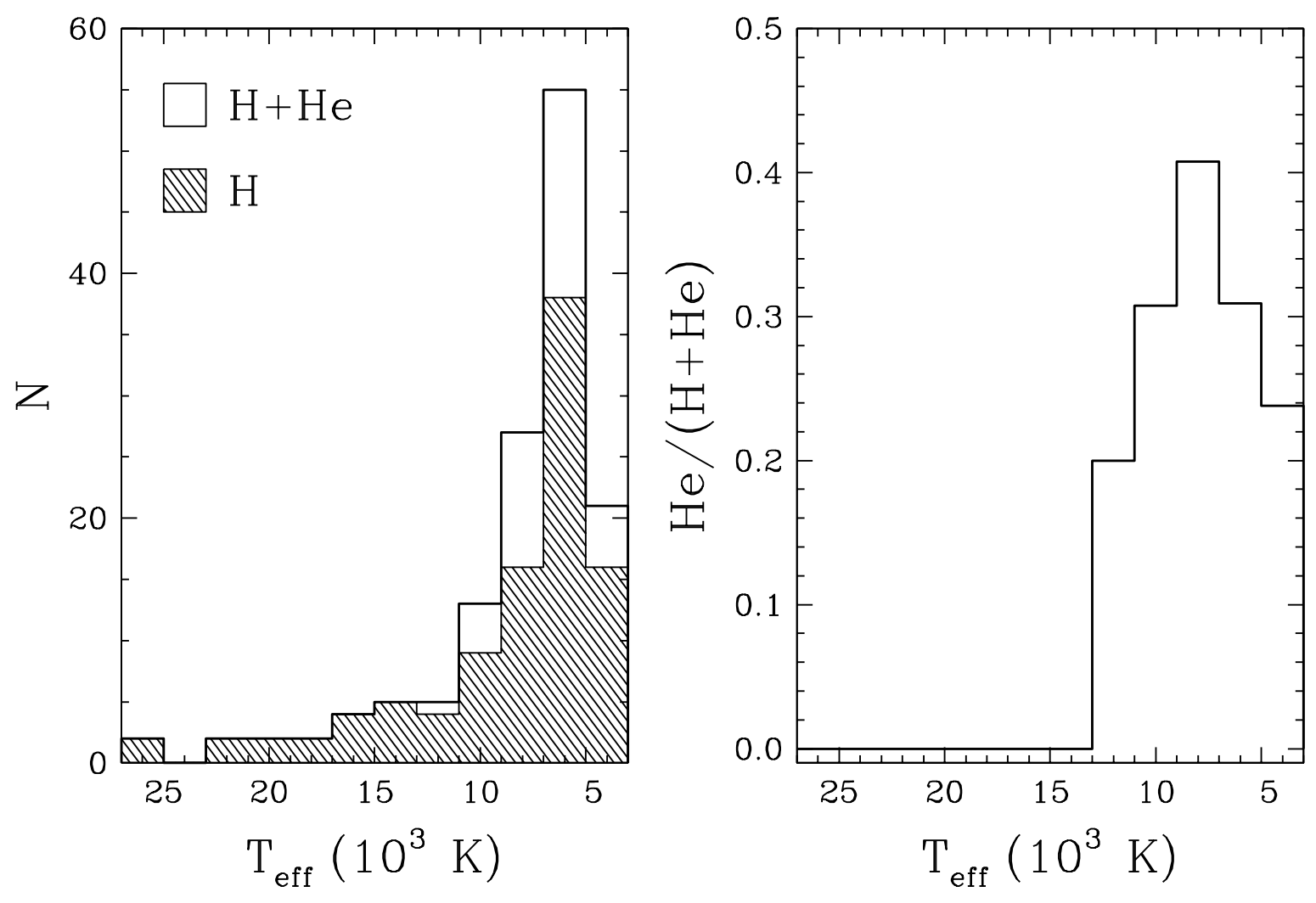

Figure 20 


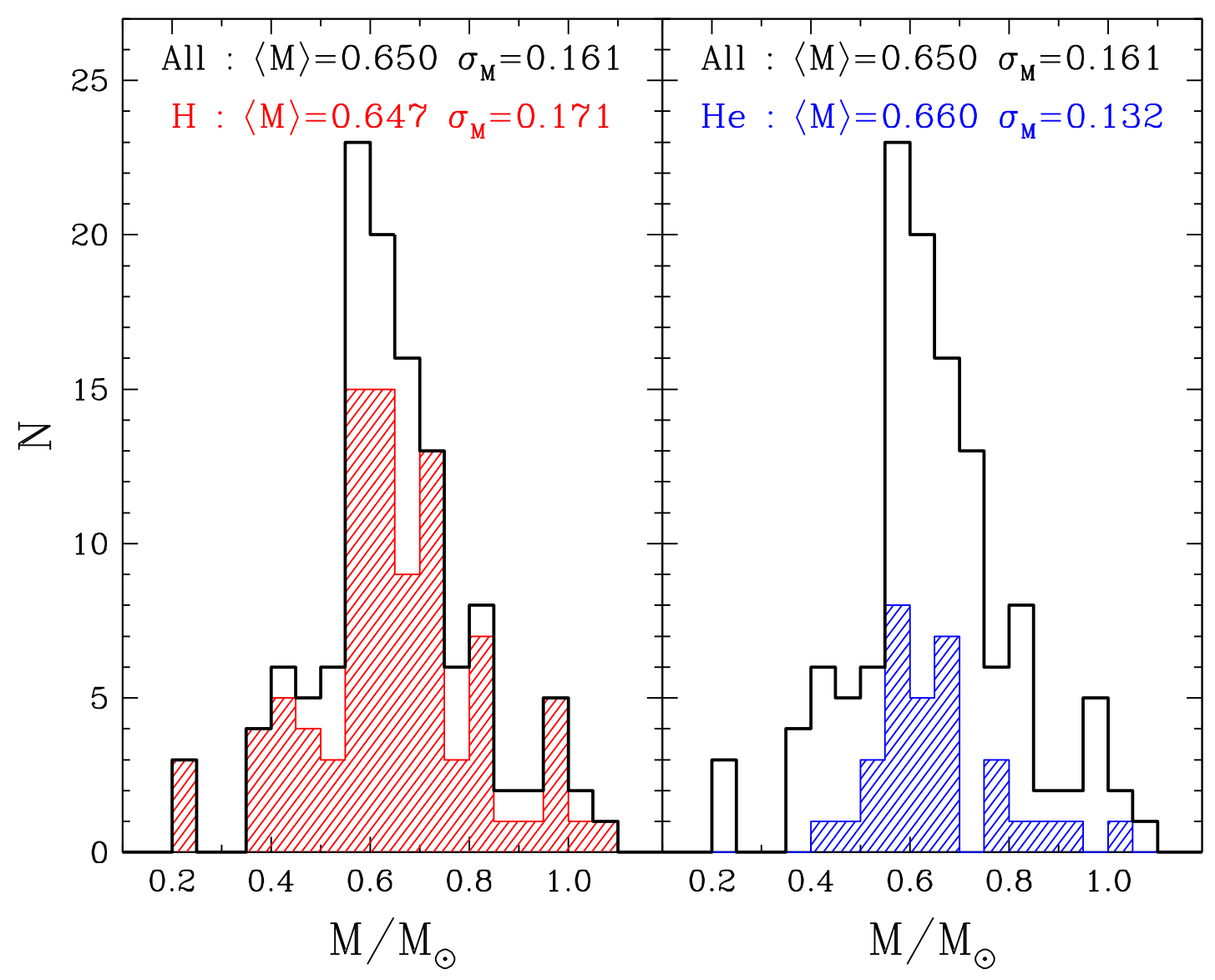

Figure 21 


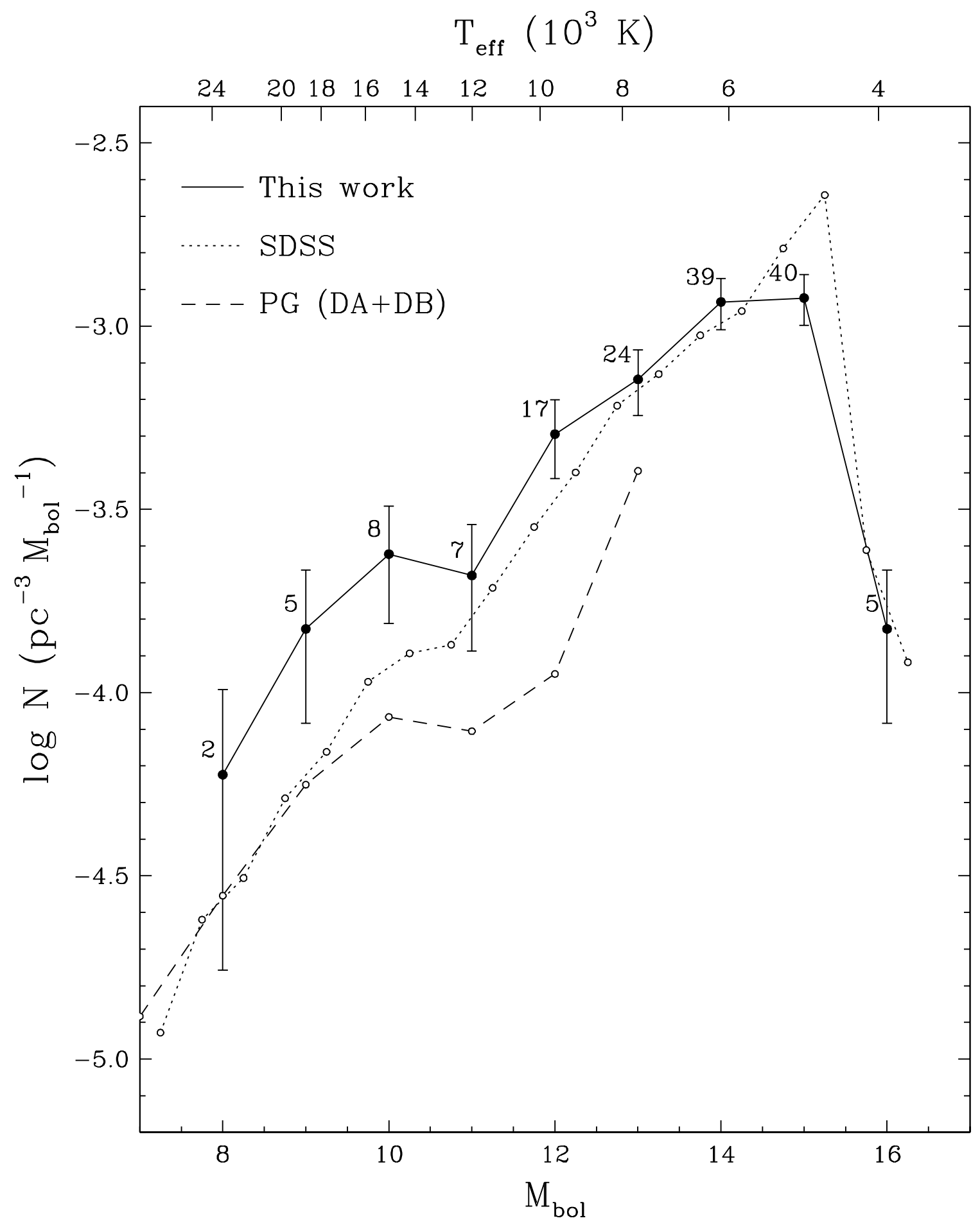

Figure 22 